\title{
ALL-SILICON CMOS PULSE-COMPRESSION NONLINEAR TRANSMISSION LINES
}

\author{
by
}

\section{Ming Li}

\begin{abstract}
A Thesis Submitted to the Faculty of Graduate Studies and Research In Partial Fulfillment of the Requirements

For the Degree of
\end{abstract}

\section{Doctor of Philosophy \\ In Electrical Engineering}

Ottawa-Carleton Institute of Electrical and Computer Engineering Department of Electronics
Carleton University
May, 2009
(a) Copyright 2009 
Library and

Archives Canada

Published Heritage

Branch

395 Wellington Street

Ottawa ON K1A 0N4

Canada
Bibliothèque et

Archives Canada

Direction du

Patrimoine de l'édition

395 , rue Wellington

Ottawa ON K1A 0N4

Canada

Your file Votre référence

ISBN: 978-0-494-52095-6

Our file Notre référence

ISBN: 978-0-494-52095-6

NOTICE:

The author has granted a nonexclusive license allowing Library and Archives Canada to reproduce, publish, archive, preserve, conserve, communicate to the public by telecommunication or on the Internet, loan, distribute and sell theses worldwide, for commercial or noncommercial purposes, in microform, paper, electronic and/or any other formats.

The author retains copyright ownership and moral rights in this thesis. Neither the thesis nor substantial extracts from it may be printed or otherwise reproduced without the author's permission.
AVIS:

L'auteur a accordé une licence non exclusive permettant à la Bibliothèque et Archives Canada de reproduire, publier, archiver, sauvegarder, conserver, transmettre au public par télécommunication ou par l'Internet, prêter, distribuer et vendre des thèses partout dans le monde, à des fins commerciales ou autres, sur support microforme, papier, électronique et/ou autres formats.

L'auteur conserve la propriété du droit d'auteur et des droits moraux qui protège cette thèse. $\mathrm{Ni}$ la thèse ni des extraits substantiels de celle-ci ne doivent être imprimés ou autrement reproduits sans son autorisation.
In compliance with the Canadian

Privacy A'ct some supporting forms may have been removed from this thesis.

While these forms may be included in the document page count, their removal does not represent any loss of content from the thesis.
Conformément à la loi canadienne sur la protection de la vie privée, quelques formulaires secondaires ont été enlevés de cette thèse.

Bien que ces formulaires aient inclus dans la pagination, il n'y aura aucun contenu manquant.

\section{Canadä}




\begin{abstract}
This thesis studies the possibility of building efficient all-silicon pulse-compression nonlinear transmission lines (NLTLs). The theory and history of NLTLs are thoroughly reviewed, and the motivation for this project and possible applications are discussed. Silicon coplanar waveguide (CPW) lines and CMOS varactors, as two key components in NLTLs, are fully researched, and were fabricated using a standard $0.18-\mu \mathrm{m}$ CMOS process. To reduce the dielectric loss caused by the conductive silicon substrate, a slowwave transmission line technique was further developed, and silicon slow-wave CPW lines were built. A measured $S_{21}$ loss of only $0.25 \mathrm{~dB} / \mathrm{mm}$ at $40 \mathrm{GHz}$ is achieved, with an effective relative permittivity as high as 25 . Six different types of varactors based on NMOS transistors were investigated. They were divided into two groups: one has monotonic capacitance-voltage curves, and the other has non-monotonic curves. This study demonstrates that the first group is more suited for single-edge, the other for double-edge pulse-sharpening. The NMOS varactors used in the final NLTL designs were fabricated and on-chip measurements up to $55-\mathrm{GHz}$ were made. NLTL transient simulations based on component measurements show a leading-edge rise time reduction of $75 \%$ for single-edge, and $60 \%$ for double-edge pulse sharpening. Following the investigation of the components, two types of NLTL circuits were designed and built on two CMOS 0.18- $\mu \mathrm{m}$ chips, one was designed for single-edge, the other for double-edge pulse compression. Large-signal measurements, based on the newly developed $X$ parameter method, show a significant compression with the second type of NLTL. However, because of its large attenuation, only minor compression is obtained with the
\end{abstract}


first type of NLTL. This research successfully demonstrates that it is possible to build pulse-compression NLTLs on low-cost commercial CMOS chips. 


\section{Acknowledgments}

I would like to acknowledge the contributions of all those who made this work possible.

My first and special thanks go to my three supervisors, Dr. Robert Harrison, Dr, Garry Tarr, and Dr. Rony Amaya. I feel lucky to have had all of them as my supervisors. They each helped me in their own unique ways. I thank Dr. Robert Harrison for his endless support of my studies and exposing me to his broad view of the microwave world. I feel privileged to have been his last $\mathrm{PhD}$ student. I thank Dr. Garry Tarr for his remarkable advice and suggestions not only regarding semiconductor theory but also on many other matters. I thank Dr. Rony Amaya for his important help based on his rich experience in CMOS chip design and measurement.

Secondly, my thanks go to the wireless group and microwave lab in the Communications Research Centre Canada (CRC), led by Dr. Valek Szwarc. I thank Carole Glaser for her generous help around the lab, and Denis Gregoire of Agilent Systems Inc. for providing the measurement instrument and help in using it. I could not have finished the testing of my chips without their help.

My greatest thanks go to my husband, Hyman Burshtyn. His non-stop support kept me going through thick and thin, and allowed me to complete this work without losing sight of family life.

This research was supported in part by the National Sciences and Engineering Research Council of Canada under grant number RGPIN 1340-2005. 


\section{Table of Contents}

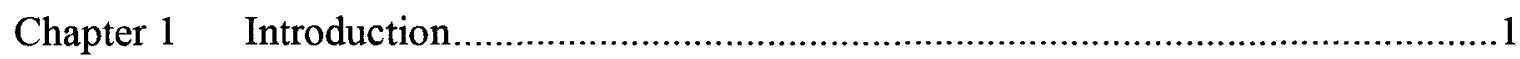

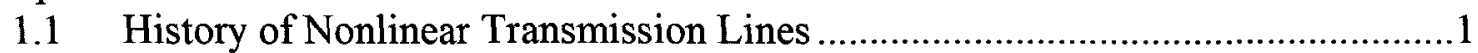

1.2 Project Motivation ...................................................................................

1.3 All-Silicon Transmission Line Pulse Compression .............................................

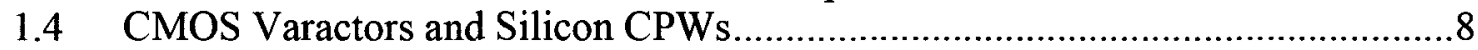

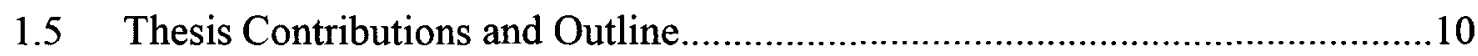

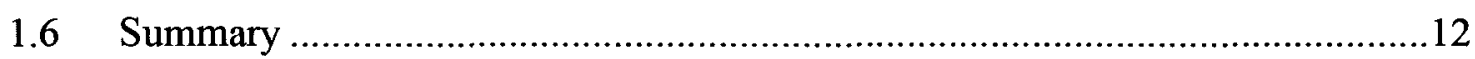

Chapter 2 Pulse Compression Technology Overview ..................................................14

$2.1 \quad$ Nonlinear Transmission Line Theory ...............................................................14

2.1.1 Dispersion and Bragg Frequency .........................................................

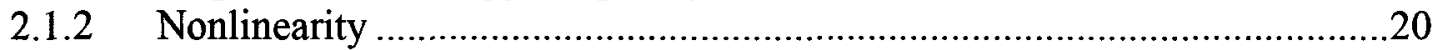

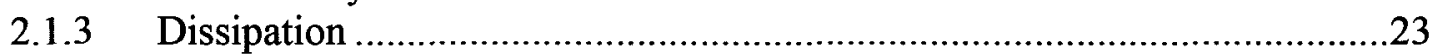

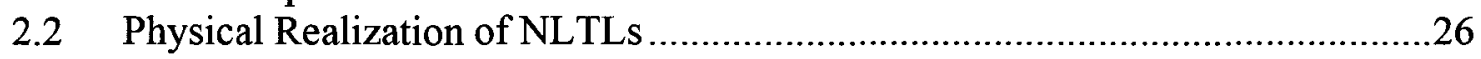

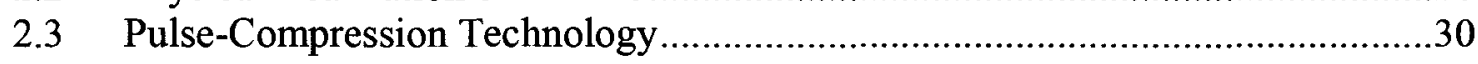

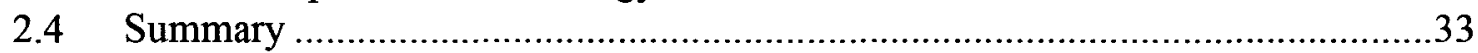

Chapter 3 Silicon CPW Performance ...................................................................34

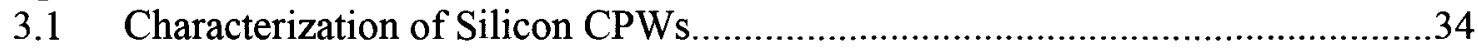

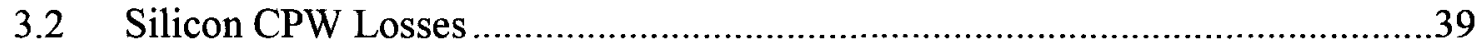

3.3 Slow-wave Technique for Substrate Loss Reduction ……...............................43

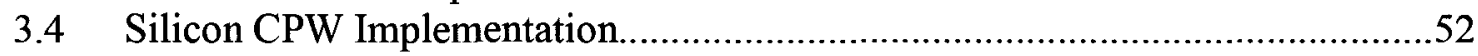

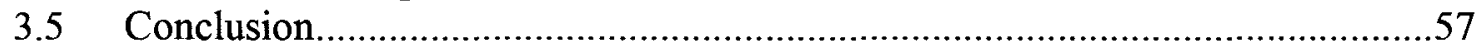

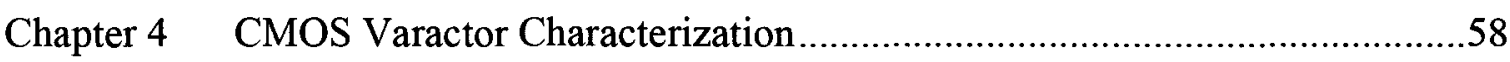

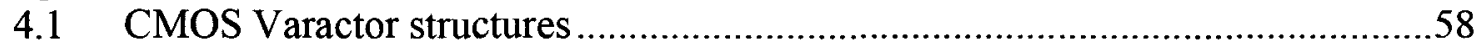

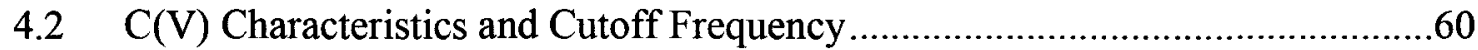

4.3 NMOS Varactor Implementation ................................................................66

4.4 High-Frequency Characteristics of CMOS Varactors.........................................69

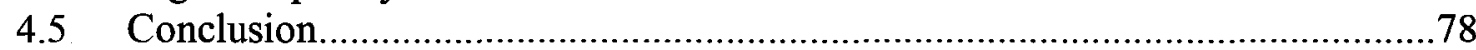

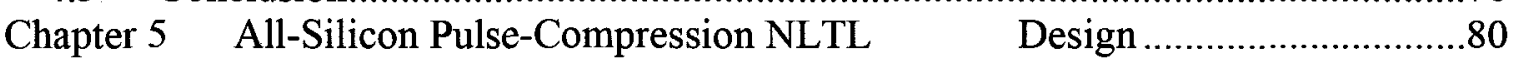

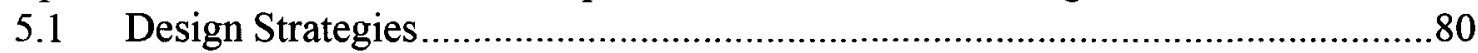

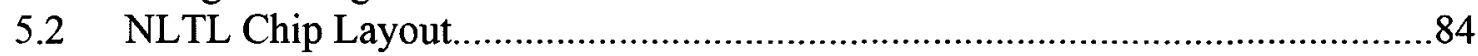

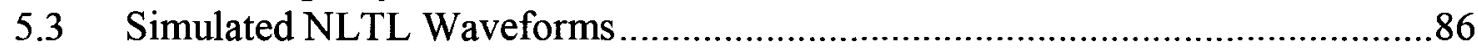

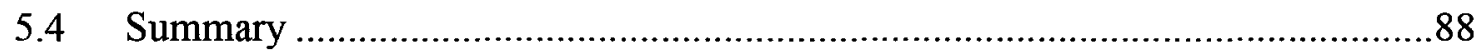

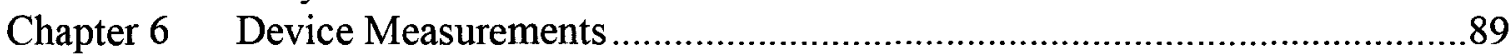

6.1 Small-signal Frequency-domain Testing ………………………....................8

6.2 Large-signal Time-domain Testing ..............................................................95

6.3 NLTL Chip Designs and Measurement Results..................................................99

6.4 Conclusion ....................................................................................

Chapter $7 \quad$ Summary and Future Directions .........................................................115

Appendix A: Propagation Characteristics of CPW Lines..........................................119

Appendix B: Calibration Methods for CMOS small-signal measurements....................122

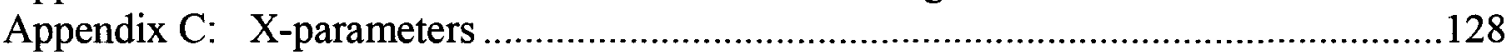

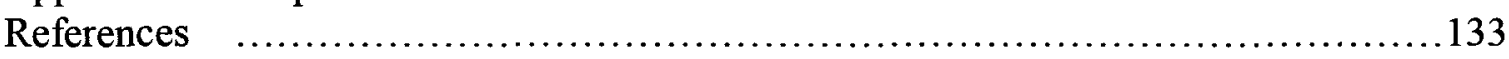




\section{List of Figures}

Figure 1-1 Distributed NLTL structures: (a) and (b) are MIS lines, (c) and (d) are

Schottky-contact lines. (a) and (c) are microstrip lines, (b) and (d) are CPW lines... 2

Figure 1-2 Periodic NLTL structures: (a) CPW on periodically doped semiconductor,

(b) Transmission line periodically loaded with varactor diodes............................. 3

Figure 2-1 Circuit diagram of the NLTL configuration. ......................................... 15

Figure 2-2 Equivalent circuit of the NLTL configuration. ......................................... 15

Figure 2-3 (a) Unit section of an NLTL, (b) its equivalent circuit. ............................. 16

Figure 2-4 Dispersion diagram for an NLTL unit cell............................................ 18

Figure 2-5 Characteristic impedance as a function of frequency for an NLTL cell....... 20

Figure 2-6 MOSFET RF Equivalent Circuit........................................................ 21

Figure 2-7 Diagram of layout-produced parasitic inductances and capacitances for a

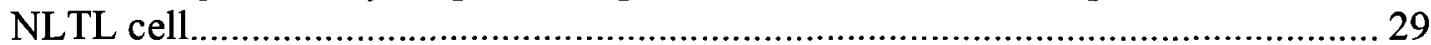

Figure 3-1 Silicon CPW structure and the field distribution. ....................................... 34

Figure 3-2 The cross-section view of a silicon CPW with pertinent dimensions........... 35

Figure 3-3 Quasi-TEM equivalent circuit of a silicon CPW: (a) Shibata's model, (b)

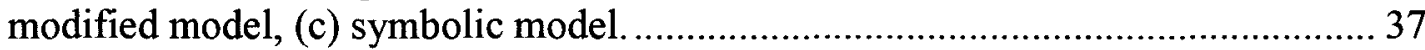

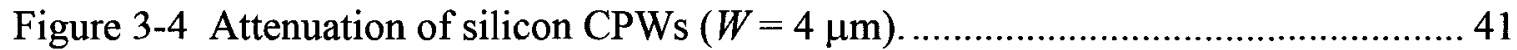

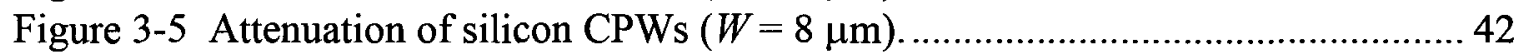

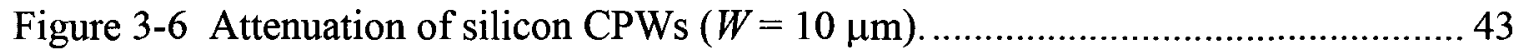

Figure 3-7 Slow-wave structure and design parameters. .......................................... 45

Figure 3-8 EM field distribution of (a) conventional CPW, (b) slow-wave CPW........ 46

Figure 3-9 Effect of overhang projection $O$ and spatial duty cycle on line impedance for

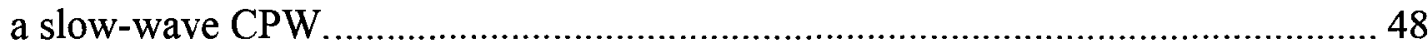

Figure 3-10 Effect of overhang projection $O$ and spatial duty cycle on line attenuation

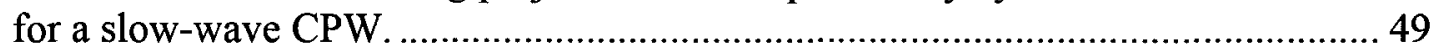

Figure 3-11 HFSS geometry for a slow-wave CPW simulation. M5 and M6 denote

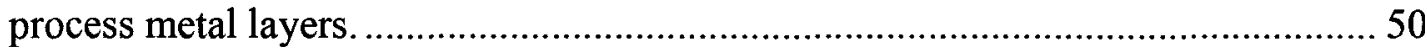

Figure 3-12 E-field distribution of a conventional CPW ..................................... 51

Figure 3-13 E-field distribution of a slow-wave CPW ............................................ 52

Figure 3-14 Technology stack for the CMOS $0.18-\mu \mathrm{m}$ process................................... 53

Figure 3-15 CMOS $2.0 \times 1.5 \mathrm{~mm}^{2}$ chip with CPW, S-CPW and NMOS varactor

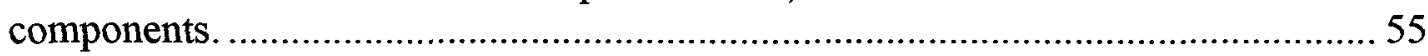

Figure 3-16 Comparison between the measured (-) and simulated (--) $\left|\mathrm{S}_{21}\right|$ for a

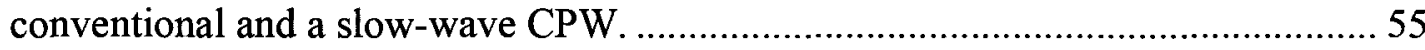

Figure 3-17 Effective relative-permittivity $\varepsilon_{r, a f f}$ for a conventional and a slow-wave

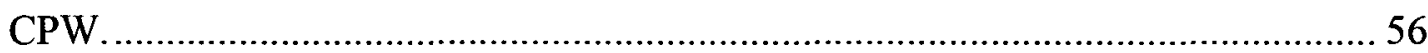

Figure 4-1 Cross-sections of six varactor structures............................................ 59

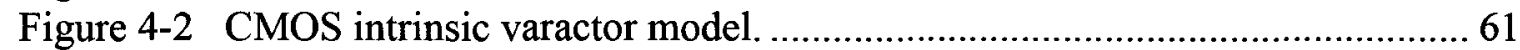

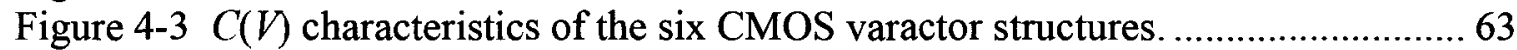

Figure 4-4 $f_{c}(V)$ characteristics of the six CMOS varactor structures...........................66 66

Figure 4-5 Measured and simulated $C(V)$ curves of (a) an SDF varactor and (b) an SDB

varactor. 
Figure 4-6 The CMOS varactor measurement and de-embedding setup...................... 71

Figure 4-7 Simulated and measured data for the AMOS varactor............................... 72

Figure 4-8 Simulated and measured data for the IMOS varactor. ................................. 72

Figure 4-9 Simulated and measured data for the NMOS varactor............................... 73

Figure 4-10 Simulated and measured data for the $\mathrm{SnDp}$ varactor............................ 73

Figure 4-11 $\mathrm{SnDp}$ varactor versus NMOS varactor at $55 \mathrm{GHz}$ (short-gate $=0.2 \mu \mathrm{m}$, long-

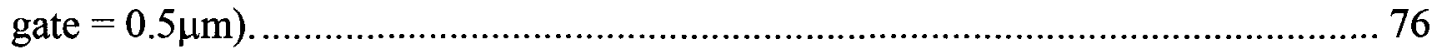

Figure 4-12 Series resistances at $5 \mathrm{GHz}$ for AMOS, SDF and SDB varactors with gate

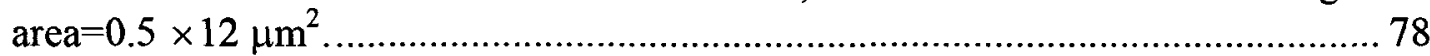

Figure 5-1 Flowchart of the silicon NLTL design process. ..................................... 82

Figure 5-2 Top view of a CMOS NLTL layout (not to scale)................................. 85

Figure 5-3 Cross-sectional view of the CMOS NLTL shown in Figure 5-2 at plane a-a'

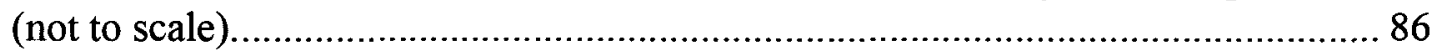

Figure 5-4 Simulated slow-wave-NLTL waveforms using measured S-CPW and varactor

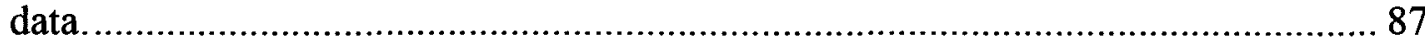

Figure 6-1 Principle of operation of a VNA........................................................ 90

Figure 6-2 On-wafer probing station connected to a VNA......................................... 91

Figure 6-3 (a) An on-wafer GSG probe. (The inset shows a microscopic view of the probe tips) (b) An artist's view of a probe (black) contacting a CPW structure (grey).

Figure 6-4 NL TL time-domain measurement setup using an oscilloscope................... 92

Figure 6-5 Simplified configuration of an NVNA and ADS workbench for nonlinear

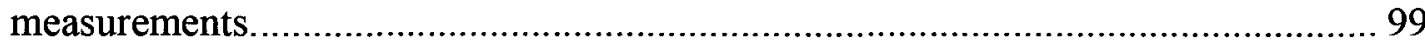

Figure 6-6 Early NLTL designs that omit slow-wave CPW and tapered-line techniques.

Figure 6-7 The latest NLTL designs, which use both slow-wave CPW and tapered-line

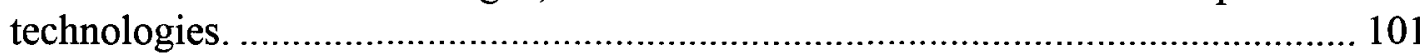

Figure 6-8 Measured SDB NLTL time-domain input (A1, yellow) and output (B2, blue) waveforms for a $13.0 \mathrm{dBm}$ input at $5 \mathrm{GHz}$................................................ 102

Figure 6-9 Measured frequency-domain SDB NLTL spectrum for a $13.0 \mathrm{dBm}$ input at 5

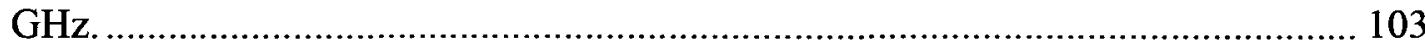

Figure 6-10 Measured SDB NLTL input (A1, yellow) and output (B2, blue) time-domain waveforms for a $13.5 \mathrm{dBm}$ input at $5 \mathrm{GHz}$.............................................. 104

Figure 6-11 Measured frequency-domain SDB NLTL spectrum for a $13.5 \mathrm{dBm}$ input at

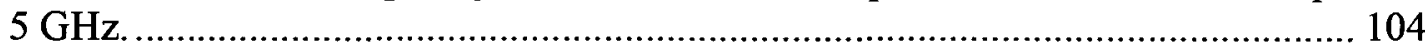

Figure 6-12 Measured SDB NLTL input (A1, yellow) and output (B2, blue) timedomain waveforms for a $13.9 \mathrm{dBm}$ input at $5 \mathrm{GHz}$....................................... 105

Figure 6-13 Measured frequency-domain SDB NLTL spectrum for a $13.9 \mathrm{dBm}$ input at

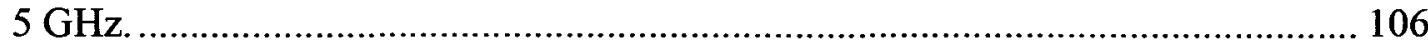

Figure 6-14 Measured SDF NLTL input (A1, yellow), reflected (B1, purple), and output (B2, blue) time-domain waveforms for a $13.0 \mathrm{dBm}$ input at $3 \mathrm{GHz}$................... 107

Figure 6-15 Measured frequency-domain SDF NLTL spectrum for a $13.0 \mathrm{dBm}$ input at 3 $\mathrm{GHz}$

Figure 6-16 Measured SDF NLTL input (A1, yellow), reflected (B1, blue), and output (B2, purple) time-domain waveforms for a $14.0 \mathrm{dBm}$ input at $4.5 \mathrm{GHz}$. 
Figure 6-17 Measured frequency-domain SDF NLTL spectrum for a $14.0 \mathrm{dBm}$ input at $4.5 \mathrm{GHz}$. 109

Figure 6-18 The SDB NLTL simulated result with $5 \mathrm{GHz}$ sinusoid input, $2.2 \mathrm{~V}$ amplitude. 110

Figure 6-19 The SDB NLTL simulated result with $5 \mathrm{GHz}$ sinusoid input, $2.3 \mathrm{~V}$ amplitude..

Figure 6-20 The SDB NLTL simulated result with $5 \mathrm{GHz}$ sinusoid input, $1.9 \mathrm{~V}$ amplitude

Figure 6-21 The SDB NLTL simulated result with $5 \mathrm{GHz}$ sinusoid input, $1.6 \mathrm{~V}$ amplitude.

Figure 6-22 Reconstructed waveform by using only 5 lowest output harmonics. 113

Figure 6-23 Spectrum of an input signal and 5 output harmonics. 113

viii 


\section{Author's Publications in Related Areas}

[1] Ming Li, Rony E. Amaya, Jean-Marc Duchamp, Philippe Ferrari, Robert G. Harrison, and N. Garry Tarr, "Low-Loss Low-Cost All-Silicon CMOS NLTLs for Pulse Compression", IEEE MTT-S International Microwave Symposium, pp. 449-452, Honolulu, Hawaii, June, 2007.

[2] Ming Li, Robert G. Harrison, Rony E. Amaya, Jean-Marc Duchamp, Philippe Ferrari, and N. Garry Tarr, "CMOS Varactors in NLTL Pulse-Compression Applications", Proceedings of the 37th European Microwave Conference, pp. 1405-1408, Munich, Germany, Oct., 2007.

[3] Rony E. Amaya, Ming Li, Robert G. Harrison, and N. Garry Tarr, "Coplanar Waveguides in Silicon with Low Attenuation and Slow Wave Reduction", Proceedings of the 37th European Microwave Conference, pp. 508-511, Munich, Germany, Oct., 2007.

[4] Ming Li, Rony E. Amaya, Robert G. Harrison, and N. Garry Tarr, "Investigation of CMOS Varactors for High-GHz-Range Applications", Accepted by the journal Research Letters in Electronics, May 2009.

[5] Ming Li, K. Krishnamurthi and R. G. Harrison, "A Fully Distributed Heterostructurebarrier Varactor Nonlinear-transmission Line Frequency Multiplier and Pulse Sharpener", IEEE Trans. Microwave Theory Tech. vol. 46, No. 12, pp. 2295 -2301, Dec. 1998.

[6] Ming Li and R. G. Harrison, "A Fully-distributed heterostructure-barrier-varactor nonlinear transmission-line Frequency Tripler", IEEE MTT-S Digest, pp. 1639 - 1642, Baltimore, June 1998.

[7] L. Roy, Ming Li, S. Labonte and N. R. S. Simons, "Measurement Techniques for Integrated-Circuit Slot Antennas", IEEE Trans. Instrumentation and Measurment, vol. 46, No. 4, pp. 1000 - 1004, Aug. 1997.

[8] L. Roy, Ming Li, "Measurement Techniques for Millimeter-Wave Integrated Antennas", IEEE Instrumentation and Measurement Technology Conference, pp. 1385 - 1390, Brussels, Belgium, June 1996.

[9] L. Roy and Ming Li, "Integrated LNA/Antennas for $30 \mathrm{GHz}$ Receivers", ANTEM'96, pp. 559 - 562, Montreal Canada, Aug. 1996.

[10] L. Roy, S. Labonte, Ming Li, "Microstrip Discontinuity Modeling for High-Speed Intcrconnccts", Canadian Conference on Electrical and Computer Engineering, Montrcal Canada, Sep. 1995.

[11] Ming Li, Q. J. Zhang and M. Nakhla, "Finite Difference Solution of EM Fields by Asymptotic Waveform Techniques", IEE Journal, Microwave, Antennas Propagations, vol. 143, No.6, pp. 512 - 520, Dec. 1996. 
[12] Ming Li, Q. J. Zhang and M. Nakhla, "Finite Difference Solution of EM Fields by Asymptotic Waveform Techniques", International Symposium on Signals, Systems and Electronics (URSI), San Francisco U.S.A. Oct. 1995.

[13] Ming Li, H. H. Tian, Y. H. Li and Y. S. Wu, "The Omni-Automatic Design of MIC MES FET Amplifiers", ACTA ELECTRONICA SINICA, vol. 24, No.2, pp. 83 - 85, Feb. 1996.

[14] J. H. Cao, Y. H. Li and Ming Li, " The Determination of Equiripple Gain Function of Broadband Matching Networks", Journal of Microwaves, vol.11, No.4, pp. 285 - 292, Dec. 1992.

[15] J. H. Cao, Ming Li and Y. S. Wu, " Performance Analysis and Automatic Design of Microwave Solid-state Feedback Amplifiers", Proceedings of China 11th Conference on Circuits and Systems, pp. 327 - 338, Guilin China, June 1993.

[16] Ming Li, Y. S. Wu, "Analytic Analysis and Synthesis of Semi-uniform FrequencyDependent Lossy Broadband Matching Networks", IEEE Proceedings of International Conference on Circuits and Systems, China, part 1, pp. 88 - 91, Shenzhen China, June 1991.

[17] Ming Li and Y. S. Wu, "Reliability and Synthesis Methods of Semi-uniform Frequency- Dependent Lossy Broadband Matching Networks", Journal of Tianjin University, No. 2, pp. 1 - 6, 1992.

[18] Ming Li, "Analytic Theory and Synthesis of Frequency-Dependent Lossy Broadband Matching Networks", M. S. Thesis, Tianjin University, 1989. 


\section{Chapter 1 Introduction}

\subsection{History of Nonlinear Transmission Lines}

Nonlinear transmission lines (NLTLs) are typically implemented as waveguides which are continuously or periodically loaded with diodes. The waveguides can be microstrip lines or coplanar waveguides (CPWs). The diodes act as voltage-dependent capacitors (varactors). NLTLs loaded continuously with diodes are called distributed or homogeneous NLTLs, while NLTLs loaded periodically with diodes are called periodic or discrete NLTLs.

Research on NLTLs began as early as the 1960's. In 1967, Guckel et al. discussed basic metal-insulator-semiconductor (MIS) microstrip lines [1], where an oxide layer insulates the semiconductor wafer from the metallic conductors. Later on, Schottkycontact microstrip lines were developed. In these structures, the insulating region arises from the depletion layer of the Schottky barrier. The depth of the depletion layer depends on the applied voltage. The nonlinearity of the lines is produced by the voltage-dependent depletion-layer capacitance. Well-known researchers in this area include Jäger [2] and Hughes [3, 4]. In 1977, Hasegawa introduced MIS and Schottky coplanar striplines on GaAs substrate [5]. Subsequently, different theoretical approaches were established, including Aubourg's finite-element analysis of lossy 
microstrip NLTLs [6], Fukuoka's analysis of slow-wave coplanar waveguide [7], and Sorrentino's study on characteristics of MIS coplanar waveguides [8]. These early developed NLTLs were all distributed NLTLs. Fig. 1-1 shows both MIS and Schottky-contact distributed NLTLs, the upper row being MIS lines and the lower row Schottky-contact lines. Also, in Fig.1, (a) and (c) are microstrip lines, (b) and (d) are coplanar waveguide (CPW) lines. An advantage of the distributed NLTLs is that they can reduce the dimension of integrated devices due to their slow-wave properties. A disadvantage is that their attenuation is high due to longitudinal current loss [9].

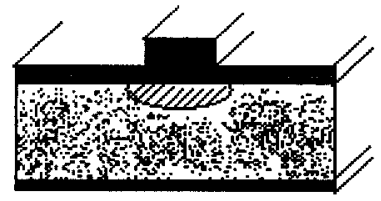

(a)

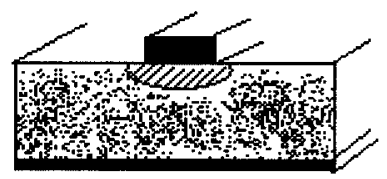

(c)

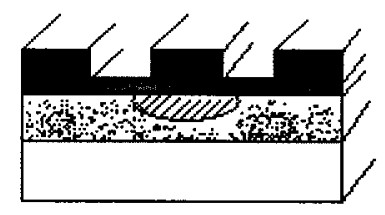

(b)

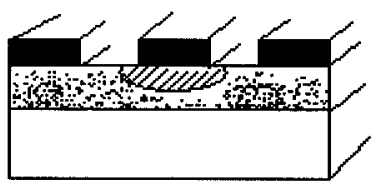

(d)

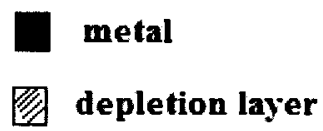

insulator

semiconductor

$\square$ semi-insulator

Figure 1-1 Distributed NLTL structures: (a) and (b) are MIS lines, (c) and (d) are Schottkycontact lines. (a) and (c) are microstrip lines, (b) and (d) are CPW lines.

The first periodic nonlinear transmission line was mentioned by Jäger in 1987 [10]. Bastida and Donzelli also introduced transmission lines loaded periodically with 
lumped nonlinear elements a year later [11]. This is a basic model for nonlinear transmission lines. In 1983, Fukuoka and Itoh proposed a novel periodic coplanar waveguide in order to overcome the large attenuation of the homogeneous strip lines [12 ]. Some examples of periodic structures are shown in Fig. 1-2. In general, compared with homogeneous NLTLs, discrete structures have much less attenuation and larger characteristic impedances. Discrete NLTLs also have a low-pass filter property, due to the Bragg cutoff frequency generated by the periodic structure [9]. This will be discussed in more detail later.

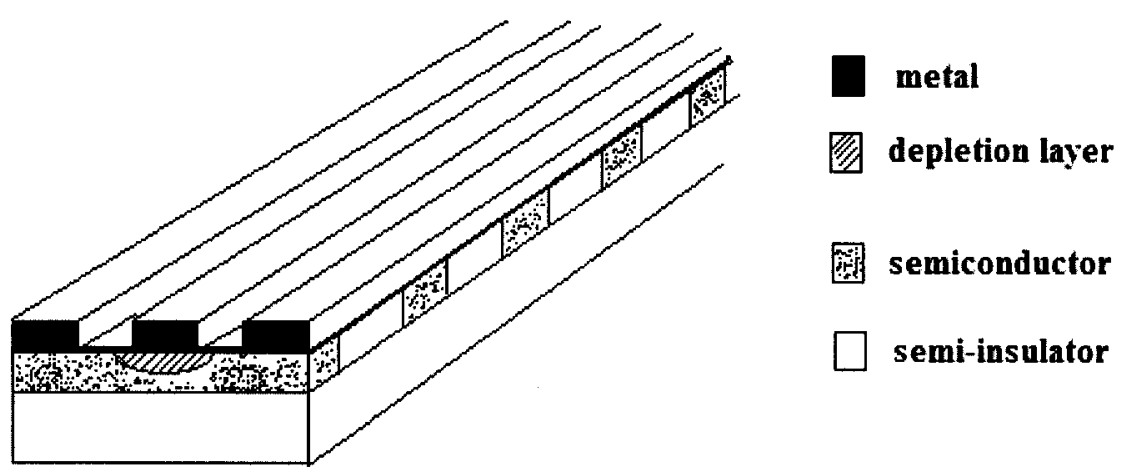

(a)

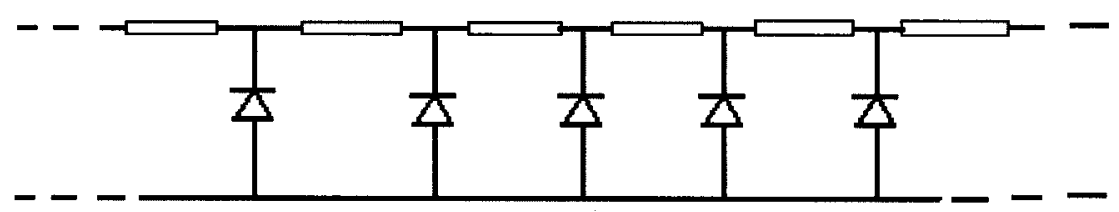

(b)

Figure 1-2 Periodic NLTL structures: (a) CPW on periodically doped semiconductor, (b) Transmission line periodically loaded with varactor diodes. 
Since the 1990's NLTLs, especially periodic ones, have become very popular, and have been used in many applications. These include pulse generation, pulse compression, and frequency multiplication. The general principle of periodic NLTLs is that the non-linear elements generate higher harmonics, and the periodic structure of the NLTL leads to a phase coupling of these harmonic signals. The pioneering researchers in this area are Jäger [13] and Rodwell [20].

\subsection{Project Motivation}

The main applications of NLTLs are (a) in the generation of sharp pulses, which can be used in high-speed sampling and on-chip interconnects, and (b) in frequency multipliers [14]. When NLTLs function in the frequency-multiplier mode, the spectral power is concentrated at one particular higher harmonic, but in the pulsesharpener mode, the amplitudes and phases of the higher harmonics combine constructively to produce pulses with short rise- and/or fall-times.

Due to rapid advances in the bandwidth and speed of electronic systems, ultrafast electronic and optoelectronic devices are currently in high demand for measuring transient electrical signals with rise times in subpicosecond range. In these measurements, picosecond or subpicosecond pulses need to be generated for signal detection in high-speed sampling circuits and frequency down-conversion in wide- 
band instruments. The NLTLs used in sampling circuits and time-domain reflectometry (TDR) act as pulse or stimulus generators. A few particular examples of those applications are generation of high-power microwave bursts in picosecond and subpicosecond measurements [15], generation of a shock wave in time-domain reflectometers [16], and generation of ultra-short electromagnetic pulses in $\mathrm{THz}$ spectroscopes [17]. Picosecond or subpicosecond electrical pulses can also be produced by optoelectronic switches through optical-electrical conversion of optical pulses from pulsed lasers, but this application is limited by the size and complexity of the lasers.

The other application of NLTLs is in on-chip interconnects. With rapidly increasing operation frequencies and growing chip sizes, global interconnects have become the top challenge in CMOS technology. Clock signals have already been brought into the multigigahertz range, resulting in the inductance and other transmission line effects of long lines, such as dispersion and skin loss, becoming the new concerns. At higher operating frequencies, as input pulses travel down a transmission line they broaden due to dispersion, and amplitudes diminish due to skin loss and substrate attenuation. The already power hungry global clock network is set to consume more power and hence become even more difficult to design. Other global interconnects, such as data bus and control lines, face similar complications. NLTLs can be used as interconnects to perform pulse compression or pulse sharpening and to overcome pulse broadening and attenuation [21]. 
Homogeneous NLTLs using a sequence of identical sections can generate shockwave or re-shape pulse signals, but require bandpass filters and phase shifters to adjust harmonic phases. Nonhomogeneous NLTLs using tapered sections can generate pulse signals or solitons from sinusoidal inputs without the need of bandpass filters and phase shifters [18]. In other words, nonhomogeneous NLTLs can transform sinusoidal signals into square waves. This capability can be used in a class-F power amplifier design, where an NLTL can be used at the input of an amplifier or as an interconnect between an amplifier and its load to produce square waves, which in turn creates nonoverlapping output voltages and current waveforms, thereby increasing the efficiency and output power [19]. This project focuses on simple and compact nonhomogeneous NLTLs.

This program also focuses on building all-silicon NLTLs in a standard CMOS process, due to its low cost, high yield, short fabrication cycle, and its compatibility with other RF circuitry. More details on this subject will be given in the next section.

\subsection{All-Silicon Transmission Line Pulse Compression}

NLTLs have received considerable attention for application in high-speed sampling, pulse regeneration [ 20$]$, integrated-circuit interconnects [21], and measurement circuitry [20]. In such applications, pulse compression and pulse 
shaping are of critical importance. As input pulses travel down a transmission line they broaden due to dispersion. NLTLs can be used to overcome pulse broadening and attenuation caused by dispersion and frequency-dependent losses. Previous NLTLs have been mostly designed using conventional microstrip or CPW transmission lines (TLs) loaded either with GaAs Schottky diodes [20], [22] or with heterostructurebarrier varactors [22]. Recently, attention has turned to the possibility of designing allsilicon NLTLs in which both the TLs and the varactors are realized in silicon. The possibility of using CMOS varactors in NLTLs was suggested in [21]. A silicon NLTL of this kind, using a BiCMOS process on a high-resistivity silicon substrate, was investigated in [23]. Another approach is to exploit silicon Schottky diodes expressly designed for NLTL applications [24].

With constantly increasing frequencies in digital equipment, downscaling of semiconductor processes, and increasing demand for low-cost integrated RF components, it is necessary to develop CMOS-compatible NLTL designs that can be realized on lossy or standard low-resistivity silicon substrates. The resulting NLTLs will facilitate integration with other all-silicon digital or analog devices.

In all-silicon NLTL designs, the greatest challenge is the loss in the silicon substrate [24], [25], which contributes to losses in both the CPW and the CMOS varactors. The lower the resistivity of the silicon substrate, the higher the NLTL losses. The aim of this research is to investigate the possibility of realizing all-silicon 
CMOS pulse-compression NLTLs, especially on standard low-resistivity silicon substrates.

\subsection{CMOS Varactors and Silicon CPWs}

In general, NLTLs consist of linear transmission lines periodically loaded with nonlinear devices, such as diodes or varactors. The nonlinearity is provided by the voltage-dependent capacitance of the nonlinear devices. In this project, silicon CPWs are used as distributed linear components and CMOS varactors as lumped-element nonlinear components.

During last 10 to 15 years, silicon CMOS technology has improved dramatically. There has been a continued increase in varactor cut-off frequencies. This enables CMOS varactors to play an important role in the implementation of RF components. In the NLTL designs described in this thesis, CMOS varactors are key components. In these varactors, the gate capacitance of a MOS field-effect transistor (MOSFET) is utilized to implement the voltage-dependent reactance. This gate capacitance varies in a nonlinear fashion as the changing MOSFET dc gate bias voltage sweeps the device through its accumulation, depletion, and inversion modes of operation. By making appropriate connections among the terminals of the MOSFET, the shape of the nonlinear capacitance-voltage $(C-V)$ characteristic can be altered. The two resulting 
alternative CMOS varactor topologies can be used for different pulse-compression purposes. This subject will be discussed in more detail in Chapter 4 .

The realization of CPW transmission lines on lossy silicon substrates has been studied experimentally and theoretically in last few decades $[26,27,28,29,30$, 31]. In the earlier studies, the CPW lines were primarily fabricated on highresistivity silicon substrates, with resistivities in the $20 \Omega$-cm to $2500 \Omega$-cm range. However, such low-loss high-resistivity wafers are more expensive than standard silicon substrates and require substantial modifications to standard CMOS fabrication processes. Recent work has begun to focus on low-resistivity silicon substrates [32], [33], [34], concentrating on how to reduce the silicon substrate losses. In [32], a metal ground plane was fabricated beneath the CPW line and above the substrate so as to block electromagnetic (EM) energy from entering the lossy substrate. In [33], a bow-shaped stacked metal ground was inserted below the CPW line in order to reduce the substrate loss by shaping the electric fields under the signal line. In [34], a micromachined polymide interface layer was added between the CPW line and the substrate in order to increase the distance between the CPW layer and the substrate, thereby reducing the substrate loss. In such work, CPW insertion losses as low as $0.6 \mathrm{~dB} / \mathrm{mm}$ have been reported [33]. This thesis presents a new slow-wave silicon CPW structure, using a standard CMOS process with a substrate resistivity of only $\sim 10 \Omega-\mathrm{cm}$. A $0.2-0.3 \mathrm{~dB} / \mathrm{mm}$ insertion loss at $40 \mathrm{GHz}$ is achieved. A detailed discussion will be given in Chapter 3. 


\subsection{Thesis Contributions and Outline}

This thesis investigates the possibility of realizing NLTL pulse compression circuits in low-resistivity silicon processes. The major challenges in all-silicon CMOS NLTL design are to overcome the high-frequency losses, to structure the CMOS varactors so as to create a strong enough nonlinearity, and to implement lower losses NLTLs in standard, low cost silicon processes. The contributions of this research are expected to be the following:

1. The theoretical and analytical development of more efficient and productive strategies and methods for designing pulse-compression NLTLs by exploiting newly-developed, market-proven software design tools.

2. The investigation and implementation of six different CMOS varactor structures, three of them providing monotonic $C-V$ curves, and the other three providing non-monotonic $C-V$ curves. One type of $C-V$ characteristic is more suited for single-edge-compression, other is better for double-edgecompression NLTL designs.

3. Investigation of the feasibility of building low-loss CPWs on low-resistivity silicon substrates. This involves the development of a new silicon CPW topology, the so called "slow-wave" structure, within a standard CMOS silicon 
process. This novel type of silicon CPW has reduced losses and can be used in pulse-compression NLTLs.

4. The development of a nonlinear theory that integrates the linear components (silicon CPWs) and the nonlinear elements (the CMOS varactors) together to form NLTLs that have minimum loss and optimized pulse-sharpening capabilities.

5. New experimental techniques for characterizing NLTLs in on-chip testing. This work includes executing calibration procedures with de-embedding techniques, and developing a new strategy for large-signal, high-frequency nonlinear signal testing.

Summarizing this thesis, progress in new pulse-compression technologies is reviewed in Chapter 2. Theories of nonlinear transmission lines and electromagnetic wave propagation are also reviewed in this chapter.

Chapter 3 mainly concerns silicon CPWs. CPW properties such as losses, impedances, effective permittivity and cutoff frequencies are discussed, and some recently improved technologies are reviewed. A new structure for low-loss CPW transmission lines realized on silicon substrates is presented. 
CMOS varactor characteristics are discussed in Chapter 4 , where six different CMOS varactor structures are investigated. Four of them were implemented on standard CMOS chips. The high-frequency characteristics of these CMOS varactors are fully studied. Of these four, two particular NMOS structures are selected, one being more suited for single-edge pulse-compression, the other for double-edge pulsecompression.

Chapter 5 presents design strategies for all-silicon pulse-compression NLTLs. The complete NLTL structure is investigated, including the influence of insertion losses on the other design considerations. This chapter includes design simulation results.

Chapter 6 deals with fabrication and laboratory measurements on the NLTL chips, including testing environment and experimental setups. Methodologies, such as onwafer calibration and de-embedding techniques, are discussed. On-chip small-signal measurements of the NLTLs are presented. A newly developed large-signal testing technique, which is used for measuring the nonlinearity of the NLTLs, is investigated in detail.

Chapter 7 summarizes the project and outlines possible future work.

\subsection{Summary}


A brief history of the development of nonlinear transmission lines (NLTLs) is presented in this chapter. It includes overviews of early NLTL development, the variety of NLTL structures designed, materials used, and manufacturing processes. The motivations for designing and building all-silicon pulse-compression NLTLs are also discussed. In addition, technologies for NLTL pulse-compression are introduced in this chapter. The contributions of this work, and an outline of the dissertation, are given at the end of the chapter. 


\section{Chapter 2 Pulse Compression Technology Overview}

Nonlinear transmission lines (NLTLs) have been popular during the last three decades because of their unique functions and small size. An important application of NLTLs is in high-speed sampling and timing systems, where they can be used to sharpen either the rising or falling edge of a pulse, (or both). This shrinking of the rise and/or fall times of the pulse is often referred to as pulse shaping or pulse compression.

\subsection{Nonlinear Transmission Line Theory}

The NLTLs investigated here consist of CPW lines interleaved with NMOS varactors. Figure 2-1 shows the configuration of an NLTL. Here $V_{s}$ and $R_{s}$ characterize the pulse source, $Z_{0}$ represents the characteristic impedance of the CPW, $d$ is the section length of the $\mathrm{CPW}$, and $C(V)$ and $R_{v}$ represent respectively the nonlinear capacitance and the loss resistance of the NMOS varactor. The approximate equivalent circuit for an NLTL is given in Figure 2-2, where $L$ and $C_{0}$ are the equivalent linear inductance and capacitance per section, and $R$ represents the series resistance of the CPW. 


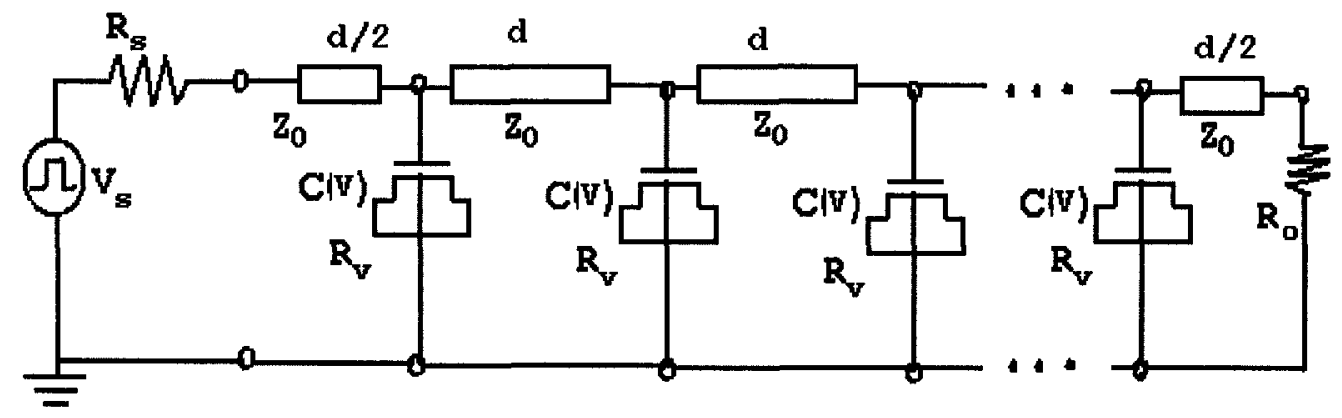

Figure 2-1 Circuit diagram of the NLTL configuration.

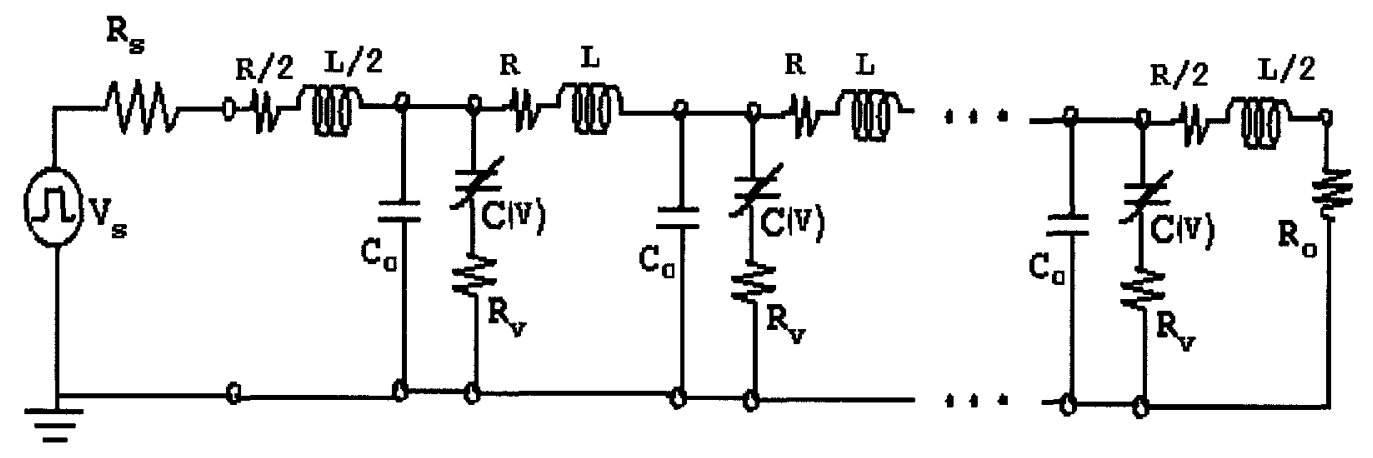

Figure 2-2 Equivalent circuit of the NLTL configuration.

A nonlinear transmission line has three fundamental and significant characteristics: nonlinearity, dispersion, and dissipation. These characteristics define an NLTL's functions and behavior. Nonlinearity is due to the voltage-dependent varactor capacitance, dispersion to the periodicity of the NLTL, and dissipation is due to the 
finite conductivity of the CPW conductors (represented by the elements $R$ in Figure 2-

2) and the series resistance $R_{v}$ of each varactor.

\subsubsection{Dispersion and Bragg Frequency}

Dispersion is variation of phase velocity with frequency. An expression for the dispersion of an NLTL can be derived from a unit cell of the line. Figure 2-3 diagrams a single section of the NLTL and its equivalent circuit.

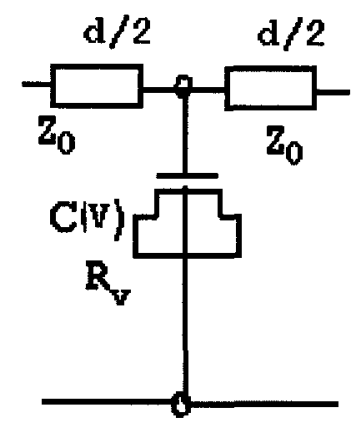

(a)

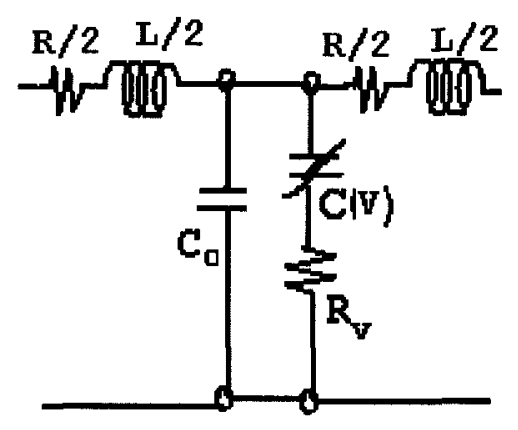

(b)

Figure 2-3 (a) Unit section of an NLTL, (b) its equivalent circuit.

To simplify the dispersion derivation, the NLTL is assumed to be lossless, that is $R$ $=0$ and $R \mathrm{v}=0$. For the equivalent circuit of the unit section, the characteristic $\mathrm{ABCD}$ matrix is 


$$
\left[\begin{array}{cc}
A & B \\
C & D
\end{array}\right]=\left[\begin{array}{cc}
1 & \frac{j \omega L}{2} \\
0 & 1
\end{array}\right] \cdot\left[\begin{array}{cc}
1 & 0 \\
j \omega C & 1
\end{array}\right] \cdot\left[\begin{array}{cc}
1 & \frac{j \omega L}{2} \\
0 & 1
\end{array}\right]
$$

where

$$
C=C_{0}+C v=C_{0}+\frac{1}{V_{H}-V_{L}} \int_{V_{L}}^{V_{H}} C(V) d V,
$$

and $V$ is the applied varactor bias voltage.

The propagation constant of the line is $\cosh (\gamma d)=(A+D) / 2$, where $\gamma=\alpha+j \beta\left(\mathrm{m}^{-1}\right)$ is the complex propagation constant, $\alpha$ and $\beta$ are the attenuation constant $(\mathrm{Np} / \mathrm{m})$ and phase constant $(\mathrm{rad} / \mathrm{m})$ respectively, while $d$ is the physical length of the NLTL. For a lossless NLTL, $\cosh (\gamma d)$ reduces to $\cos (\beta d)$. From equation (2.1), the lossless dispersion formula is found to be

$$
\cos (\beta d)=1-\frac{\omega^{2} L C}{2}
$$

or in terms of phase

$$
\beta d=\cos ^{-1}\left[1-\frac{\omega^{2} L C}{2}\right]
$$


The dispersion relationship is shown in Figure 2-4. Here it is assumed that $L=68 \mathrm{pH}$, $C=C_{0}+C_{\nu}=8.2+19 \mathrm{fF}=27.2 \mathrm{fF}$ for the equivalent circuit of a NLTL cell in Figure 2-2 (b). Here we assume small-signal operation, so that $C_{v}$ can be treated as a linear capacitance.

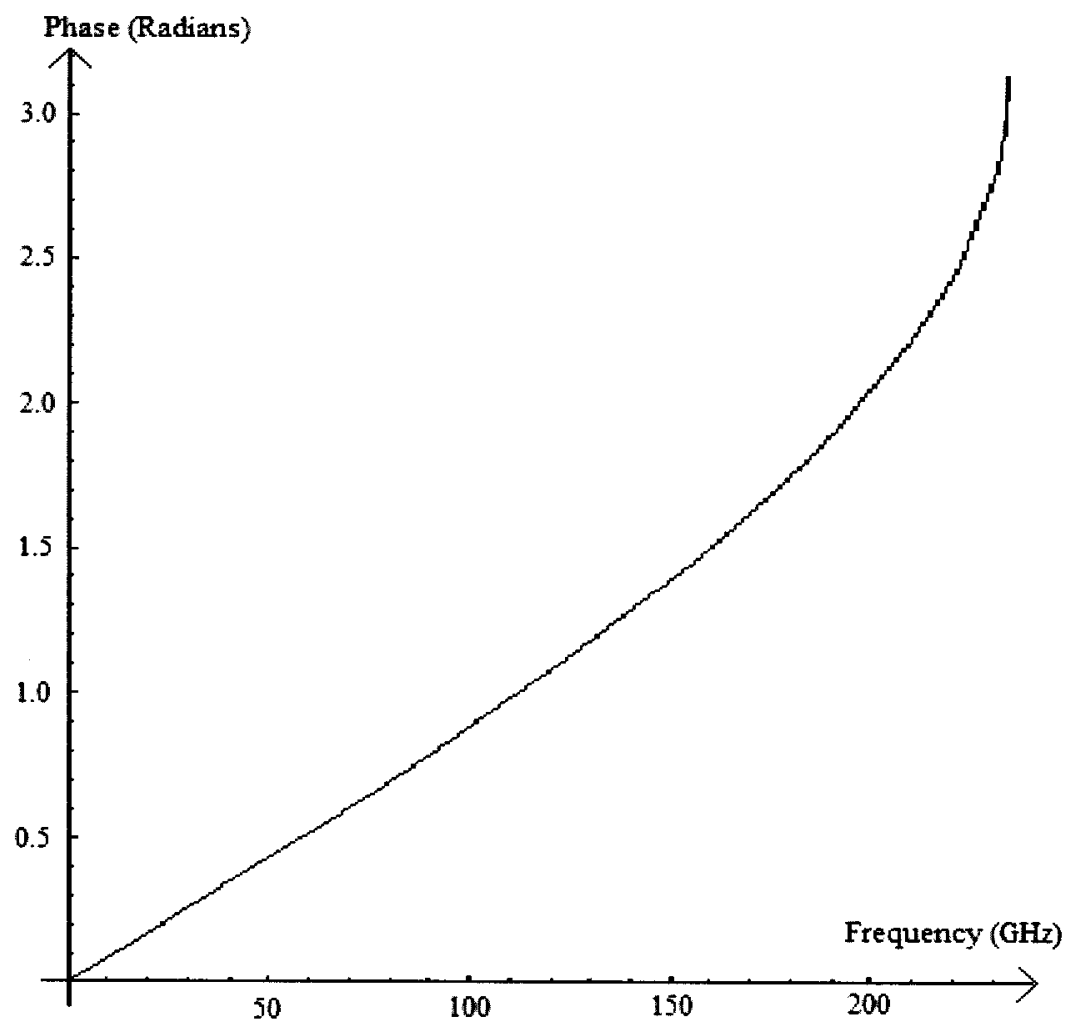

Figure 2-4 Dispersion diagram for an NLTL unit cell 
The small-signal characteristic impedance of the line is $Z=\sqrt{B / C}$. From (2.1), we have

$$
Z=\sqrt{\frac{L}{C}-\frac{\omega^{2} L^{2}}{4}} .
$$

Figure 2-5 shows the characteristic impedance $Z_{0}$ as a function of frequency. The $L$ and $C$ element values of the equivalent NLTL cell are the same as those used for Figure 2-4.

Figures 2-4 and 2-5 show that the dispersion relationship has a low-pass characteristic. The Bragg frequency $f_{\mathrm{B}}$ is defined as the low-pass corner frequency at which the propagation factor $\beta d$ is equal to $\pi$ and the impedance $Z$ becomes zero. From equation (2.2) or (2.3) we have

$$
f_{B}=\frac{1}{\pi \sqrt{L C}} .
$$

In Figure 2-5, the Bragg frequency is seen to be $230 \mathrm{GHz}$. 


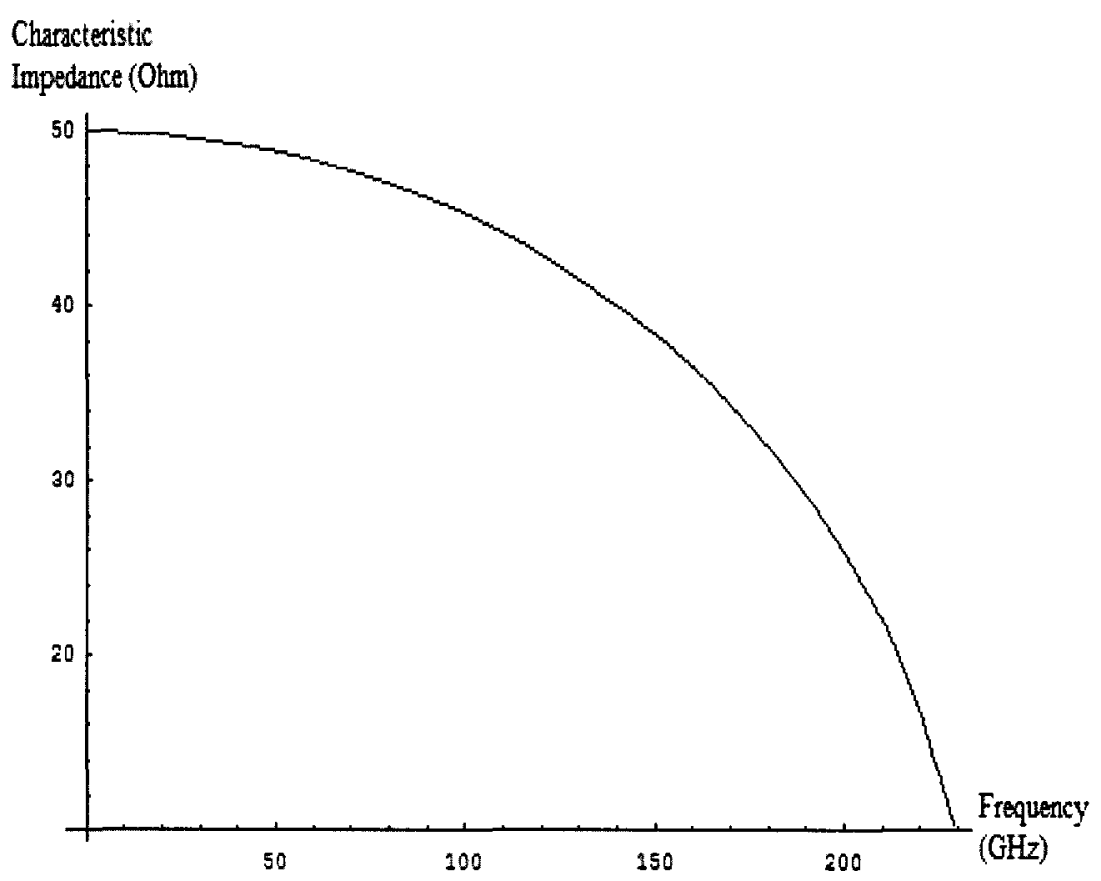

Figure 2-5 Characteristic impedance as a function of frequency for an NLTL cell.

\subsubsection{Nonlinearity}

Nonlinearity of the NLTLs is due to the nonlinear components such as diodes or varactors. There are two types of nonlinearity: conductive and reactive. Conductive nonlinearities are defined by $I(V)$ curves while reactive nonlinearities are defined by $C(V)$ curves. In mixer or detector applications nonlinear conductive elements are used. In many other RF applications, due to the dissipative nature of the conductive nonlinearity, reactive nonlinearity is much more preferred.

Figure 2-6 shows a MOSFET RF equivalent circuit [35]. 


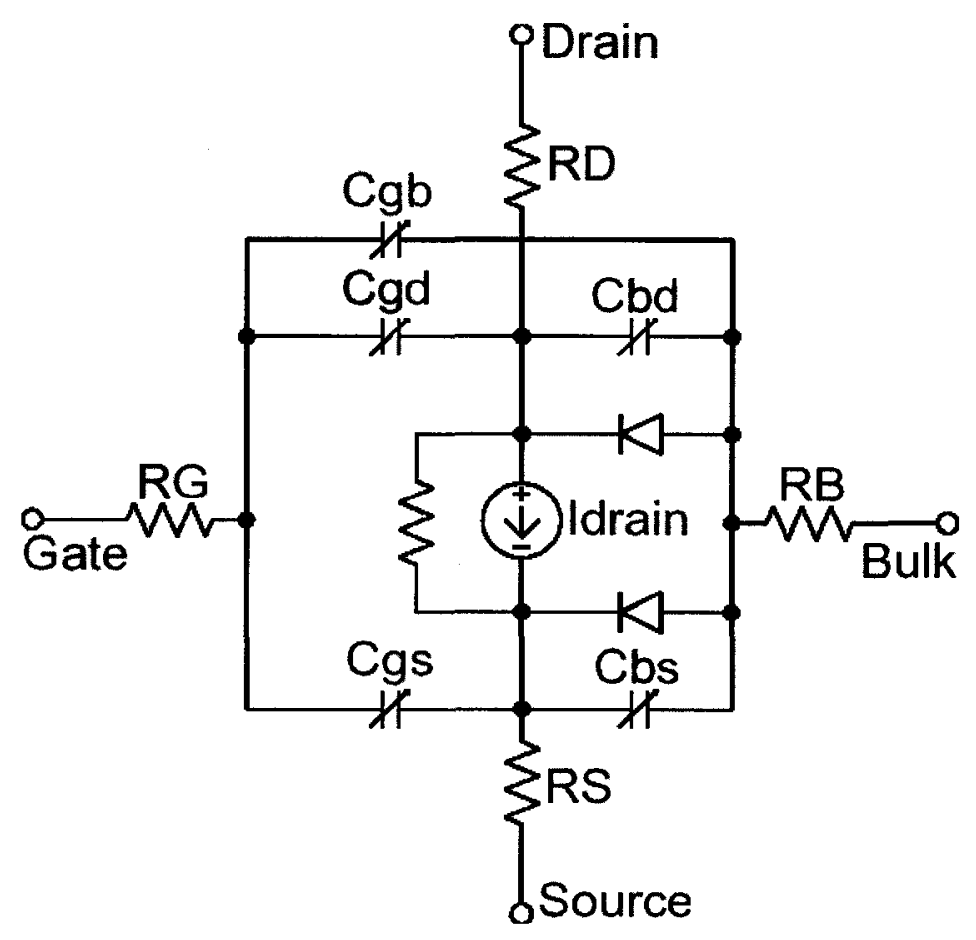

Figure 2-6 MOSFET RF Equivalent Circuit.

The shape of the $C(V)$ characteristic of a diode or varactor depends mainly on the structure of the device. Taking the total gate capacitance of a MOS transistor as example and assuming small-signal conditions, the capacitance $C_{\mathrm{gs}}$ between the gate and source is approximately given by [36]

$$
C_{g s}=\frac{2}{3} W L C_{o x},
$$


where $W$ is the gate width and $L$ is the effective gate length. $C_{o x}=K_{o x} \varepsilon_{0} / t_{0 x}$ is the gate capacitance per unit area, where $K_{o x}$ is the relative permittivity of $\mathrm{S}_{\mathrm{i}} \mathrm{O}_{2}, \varepsilon_{0}$ is free space permittivity, and $t_{o x}$ is the thickness of the thin oxide under the gate. This capacitance is primarily due to the change in channel charge as a result of a change in $V_{G S}$ (the voltage between the gate and source).

Also, the capacitance $C_{s b}$ between the source and bulk is given approximately by

$$
C_{s b}=\left(A_{s}+A_{c h}\right) \frac{C_{s j 0}}{\sqrt{1+\frac{V_{S B}}{\phi_{0}}}} .
$$

Here $A_{s}$ is the area of the source junction, $A_{c h}$ is the area of the channel, $V_{S B}$ is the applied voltage between the source and bulk, $C_{s j 0}$ is the depletion capacitance of the source junction at zero bias, and $\phi_{0}$ is the barrier potential at zero bias.

In the large-signal case, the effective gate length and the channel area will be a function of depletion depth but only a very weak function, which in turn is a function of the applied voltage $V$. The $C(V)$ curves given (2.5) and (2.6) are different for different doping profiles, and numerical methods are often required to obtain the $C(V)$ 
data. These data can be approximated by various different functional relationships. The most common function used for diode $C(V)$ curves is

$$
C_{j}(V)=\frac{C_{j 0}}{\left(1-V / V_{0}\right)^{N}}
$$

where $C_{j}$ is the junction capacitance, $C_{j 0}$ is the fitted zero-bias junction capacitance, $V$ is the junction potential, $V_{0}$ is the fitted barrier potential, and $N$ is a fitting coefficient which depends on the junction doping profile. The $C(V)$ of (2.7) fits most diode characteristics because it provides three fitting parameters: $C_{j 0}, V_{0}$, and $N$.

\subsubsection{Dissipation}

There are two main sources of dissipation in an NLTL. One is loss in the diode series resistance ( $R_{v}$ in Figure 2-3), the other is metal conductor loss ( $R$ in Figure 2-3). Diode losses are caused by the non-zero contact and bulk resistances of the semiconductor structure while metallic losses are due to the geometry and finite conductivity of the CPW.

The diode loss can be represented by 


$$
\alpha_{c-d i o}=\frac{G_{\text {shunt }}}{2 Y_{N L T L}}(\mathrm{~dB} / \mathrm{m})
$$

where $G_{\text {shunt }}$ is the shunt conductance of the diode, and $Y_{\text {NLTL }}$ is the characteristic admittance of the loaded NLTL. The diode loss increases with the square of the operating frequency.

For high-frequency CPWs built on a silicon substrate, there are more than just resistive metallic losses. The first is the conductor loss which includes metal skineffect loss. The second is the silicon substrate loss or dielectric loss, and the third is the radiation loss.

The conductor loss can be expressed as [37]

$$
\alpha_{c_{-} C P W}=8.69 \frac{\left(R_{s}+R_{g}\right)}{2 Z_{C P W}} \quad(\mathrm{~dB} / \mathrm{m})
$$

where $R_{s}$ the unit-length resistance of CPW signal line, and $R_{g}$ is the unit-length resistance of the CPW ground lines. 
The dielectric loss can be written [38]

$$
\alpha_{d_{-} C P W}=8.69 \frac{\pi}{\lambda_{0}} \frac{\varepsilon_{r}}{\sqrt{\varepsilon_{r e f f}}} \kappa \tan (\delta) \quad(\mathrm{dB} / \mathrm{m})
$$

where $\lambda_{0}$ is free space CPW guide wave length, $\varepsilon_{r}$ and $\varepsilon_{\text {reff }}$ are relative and effective permittivities of the CPW substrate, $\kappa$ is the structure coefficient of the CPW, and $\delta$ is the dielectric loss angle of the substrate.

The radiation loss can be represented by [39]

$$
\alpha_{r_{-} \mathrm{CPW}}=8.69\left(\frac{\pi}{2}\right)^{5} \frac{2\left(1-\frac{\varepsilon_{r e f f}}{\varepsilon_{r}}\right)^{2}}{\sqrt{\frac{\varepsilon_{r e f f}}{\varepsilon_{r}}}} \frac{(w+2 s)^{2} \varepsilon_{r}^{1.5}}{K\left(k_{0}^{\prime}\right) \cdot K\left(k_{0}\right)}\left(\frac{f}{c_{0}}\right)^{3} \quad(\mathrm{~dB} / \mathrm{m}),
$$

where $w$ is the width of the CPW signal line, $s$ is the gap between the CPW signal and ground lines, $k_{0}=w /(w+2 s), K\left(k_{0}\right)$ is the complete elliptic integral of the first kind, $K\left(k_{0}^{\prime}\right)=K\left(\sqrt{1-k_{0}^{2}}\right), f$ is the operating frequency, and $c_{0}$ is the free-space speed of light. 
The conductor loss is proportional to $f^{0.5}$, the dielectric loss to $f$, and the radiation loss to $f^{3}$, where $f$ is the operating frequency. These formulae only apply for NLTLs at frequencies well below the Bragg frequency. In order to determine the exact effects of diode and skin losses on the NLTL, we need to introduce the losses into the $A B C D$ matrices and extract the real part of complex propagation constant $\gamma$. Assume a transmission line with a length of $d$ and characteristic impedance of $Z_{0}$, its $A B C D$ parameters are:

$$
\left[\begin{array}{ll}
A & B \\
C & D
\end{array}\right]=\left[\begin{array}{cc}
\operatorname{Cosh}(\gamma \cdot d) & Z_{0} \cdot \operatorname{Sinh}(\gamma \cdot d) \\
\frac{1}{Z_{0}} \cdot \operatorname{Sinh}(\gamma \cdot d) & \operatorname{Cosh}(\gamma \cdot d)
\end{array}\right]
$$

The line insertion and reflection losses can be calculated respectively by [40]:

$$
S_{21}=\frac{2}{A+\frac{B}{Z_{0}}+Z_{0} \cdot C+D}
$$

$$
S_{11}=\frac{A+\frac{B}{Z_{0}}-Z_{0} \cdot C-D}{A+\frac{B}{Z_{0}}+Z_{0} \cdot C+D} .
$$

\subsection{Physical Realization of NLTLs}

The NLTLs as monolithic integrated circuits are fabricated on semiconductor substrates. NLTLs can be designed for a variety of applications, but all suffer from parasitic effects of lossy transmission lines, parasitic series resistance of diodes or 
varactors, and other parasitics that depend on the layout. What follows is a discussion of the physical nature of the NLTLs without considering the various parasitic effects. Reasons are given for choosing particular types of transmission lines, varactors, and layout structures.

In order to make an NLTL work at high-GHz frequencies, a low loss transmission line is required. Currently, there are several types of monolithically integrable transmission lines: microstrip, CPW, stripline, suspended substrate line, coplanar strips, and slotline [40]. Slotline has very low characteristic impedance, so it would be a poor choice for $50-\Omega$ systems since diode loading reduces the NLTL impedance. Coplanar strips have very high characteristic impedance, but these lines require a balanced signal. In high frequency systems, most circuits require an unbalanced signal referenced to a common ground. Striplines require symmetric dielectric material on both sides of the conductor; hence they are inappropriate for monolithic integration. Suspended substrate lines are very similar to microstrips but with lower dielectric losses. The problem with this structure is that it requires the conductors to be suspended in a grounded box, good for packaging but unsuitable for on-wafer interconnects. Therefore, only microstrips and CPWs are commonly used in monolithic integrated circuits.

Microstrips have been around for a long time [41], and there are tremendous resources available, by way of mathematical models and simulation equivalents for 
different sizes of the lines and discontinuities, and so on. Microstrip is essentially a strip of metal on an insulator that has a back plane of ground metal. This structure is ideal for inserting devices in series with the line, but in order to place an element in shunt, a via-hole must be drilled through the substrate to let the devices make a connection with the ground. Via-holes introduce parasitic inductance, fabrication difficulties, and layout problems. Even though microstrip is still the industry standard for microwave and millimeter wave circuits, CPWs have become more popular in the last decades.

Coplanar waveguide (CPW) offers convenient shunt and series element equivalents, allows a reasonable range of impedance and has relatively low losses. In this research work, CPWs are chosen as the NLTLs' linear components. Unfortunately, because there are few and inadequate $\mathrm{CPW}$ discontinuity models available, intensive electromagnetic simulations are necessary when designing such CPWs.

NLTLs' nonlinear behaviour comes from capacitive nonlinearity of diodes or varactors. Diode loss reduces NLTL nonlinear performance, as pointed out in [20]. Good diodes for NLTL applications should have low series resistances. This can be achieved by choosing proper diode or varactor structures and doping profiles. In this study, NMOS varactors are used as the NLTL nonlinear components since they are standard components in the CMOS process. Different structures of the NMOS varactors are discussed in Chapter 4. 
In high-frequency operation, it is also important to minimize discontinuities of interconnections and to reduce sources of parasitic effects associated with NLTL layouts. Figure 2-7 shows layout-produced parasitic inductances and capacitances for a NLTL cell, where the series inductance is produced by via-connections between varactors and CPWs, and the shunt capacitance is caused by metal fins used to connect the varactors to the CPW lines. These parasitics can affect NLTL functioning; they can couple energy from desired the CPW mode into other undesired modes. There are many ways to produce a good layout. Increasing via density is one way to reduce the parasitic inductance. Making a perfectly symmetric layout structure on both sides of the CPW signal line can avoid the coplanar-strip-mode (the two CPW ground planes at different potentials). Reducing unnecessary metal overhanging can reduce the parasitic capacitance. However, the layout parasitics cannot be totally eliminated. It is necessary to take them into account when doing the designing.

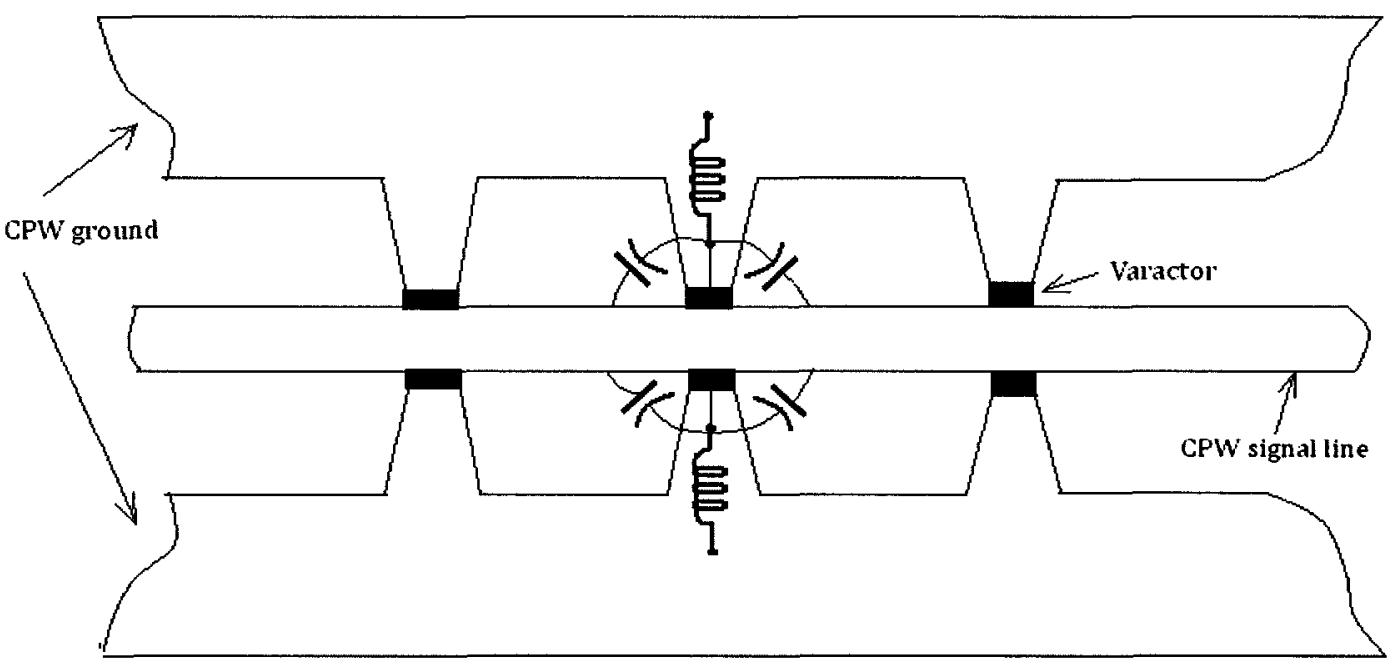

Figure 2-7 Diagram of layout-produced parasitic inductances and capacitances for a NLTL cell. 


\subsection{Pulse-Compression Technology}

Transmission lines loaded with diodes or varactors can function as pulsecompression components. Rodwell [20] contributed classical analyses of Schottkydiode CPW lines. If dispersion and losses are neglected, one need only consider the $L C$ equivalent circuit operating well below the Bragg frequency. For such an NLTL, the small-signal propagation delay decreases with increasing reverse bias voltage. For an input pulse with fall time $T_{f_{-} \text {in }}$ over a voltage swing from $V_{\text {low }}$ to $V_{\text {high }}$, this predicts the output pulse fall time to be

$$
T_{f_{-} \text {out }}=T_{f_{-} \text {in }}-l \mid\left(\sqrt{L C\left(V_{\text {high }}\right)}-\sqrt{L C\left(V_{\text {low }}\right)} \mid \quad(\mathrm{s}),\right.
$$

where $l$ is the line length, and $L$ and $C(V)$ are the inductance and voltage-dependent capacitance per unit length respectively. Theoretically, the output pulse fall time can reach to zero.

Rodwell defined the effective loading capacitance of the diode over the pulse's voltage swing (and therefore also the effective NLTL impedance.) to make it a constant. This results in the so called large-signal capacitance 


$$
C_{\text {ls }}=\frac{1}{V_{\text {high }}-V_{\text {low }}} \int_{V_{\text {high }}}^{V_{\text {lon }}} C_{j}(V) d V
$$

and characteristic impedance

$$
Z_{l s}=\sqrt{\frac{L}{C_{l s}+C_{\text {line }}}}(\Omega)
$$

where $C_{j}(V)$ is the diode capacitance, $L$ is the CPW per unit length inductance, and $C_{\text {line }}$ is the CPW per unit length capacitance.

Following Figure 2-3, where the diode equivalent circuit is $R_{v}$ in series with $C(V)$, the varactor cutoff frequency is defined as

$$
f_{c}=\frac{1}{2 \pi R_{V} C(V)}
$$

while the Bragg cutoff frequency is

$$
f_{B}=\frac{1}{\pi d \sqrt{L\left(C_{0}+C(V) / d\right)}}
$$


Here we assume that $L$ and $C_{0}$ are the per-unit-length inductance and capacitance of the CPW. In large-signal operation, $C(V)$ is replaced by $C_{l s}$.

According to [20], when $f_{c} / f_{B}$ is $4 \sim 6$, the NLTL is in a weak dispersion mode, and can produce shock waves. In other words, if we input a pulse wave, the rising or fall edge of the pulse wave at the output will be sharpened. If the $f_{c} / f_{B}$ ratio is less than 4 , the compression mode will not be exhibited by the NLTL. If the ratio is much higher than 6, the NLTL becomes more dispersive and solitons can be generated in the line. The soliton case will not be discussed in this thesis.

There are three things that can limit the NLTL pulse compression efficiency: the substrate or dielectric loss, the diode resistance, and the CPW attenuation, including the frequency-dependent skin-effect loss. The substrate loss depends on the process technique. The diode resistance loss depends on the diode's structure. The CPW loss depends on the CPW dimensions. For example, CPW skin loss can be reduced by increasing the centre conductor width of the $\mathrm{CPW}$ but at the cost of increasing the radiation loss. In general, the longer, the NLTL, the larger the attenuation. The use of the tapering technique can reduce the NLTL attenuation. Because of the nonlinear properties of the NLTLs, when a pulse signal propagates along the line, the waveform which is closer to the input end has a narrower bandwidth, with fewer harmonics, than that of the waveform further down along the line. One can use a low Bragg cutoff 
frequency at the input end and a high Bragg cutoff frequency at the output end to reduce the total length and size of the NLTL, thereby reducing its attenuation. There are two ways to taper an NLTL. One way is to taper the width of the CPW signal line, making it widest at the input end and gradually narrowing it down to the output end, as mentioned in [61]. The disadvantage of this method is that it creates CPW discontinuity and makes both the design and layout complicated. Another way is to taper the NLTL section length, that is, placing the longest section-length at the input end and gradually reducing the lengths of the sections towards the output end, as presented in [20]. This work used the second tapering method. No matter which method is used, the width of the varactors used in the NLTL has to be tapered too. One reason is to keep the right ratio between the Bragg cutoff frequency and the varactor cutoff frequency at the each section. A second reason is to keep the impedance at the each section at $50-\Omega$. More details on the tapering technique will be given in the later chapters.

\subsection{Summary}

This chapter gives an overview of NLTL pulse-compression technology. The three main characteristics of the NLTL, dispersion, dissipation, and nonlinearity, are fully described. The challenges of designing all-silicon pulse-compression NLTLs are pointed out. Solutions for overcome these challenges are suggested. The framework of physically realizing the NLTLs is also described. 


\section{Chapter 3 Silicon CPW Performance}

\subsection{Characterization of Silicon CPWs}

CPWs have become the most popular components among planar microwave transmission lines during last two decades. Figure 3-1 shows the structure of a silicon CPW (top) and its electromagnetic field distribution (bottom). A CPW line consists of one signal line and two symmetrical ground planes. This balanced structure makes it easy to fabricate CPWs on semiconductor substrates and to integrate them with other MMIC or RFIC components. As shown in Figure 3-1, some of the electric field lines produced by the signal line terminate at the ground planes, the remainder terminate at the metal ground beneath the substrate. The magnetic field lines loop around the signal line, and are always perpendicular to the electric field.

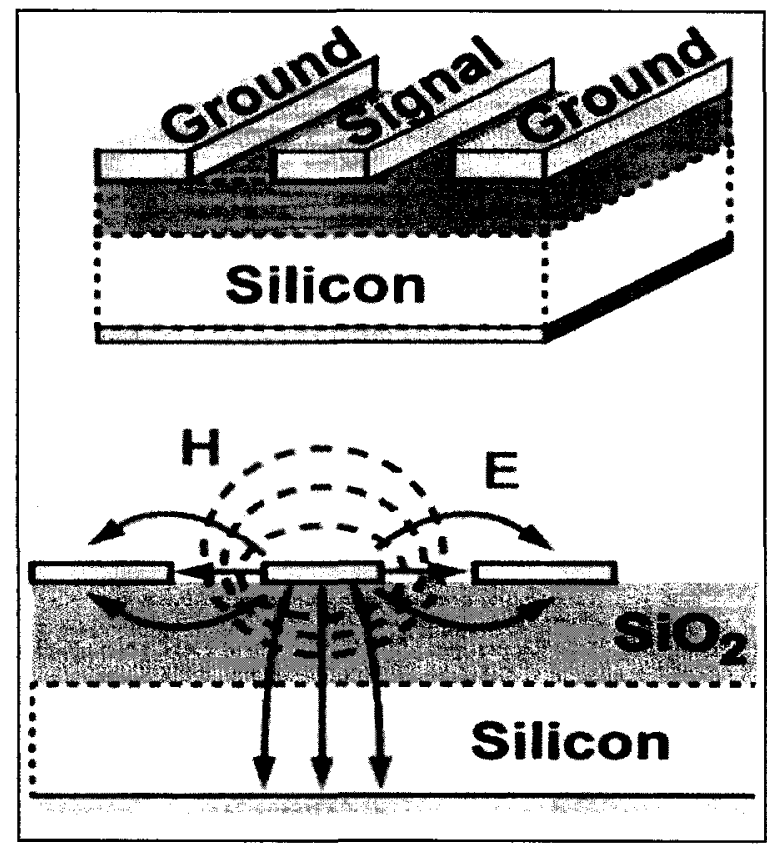

Figure 3-1 Silicon CPW structure and the field distribution. 


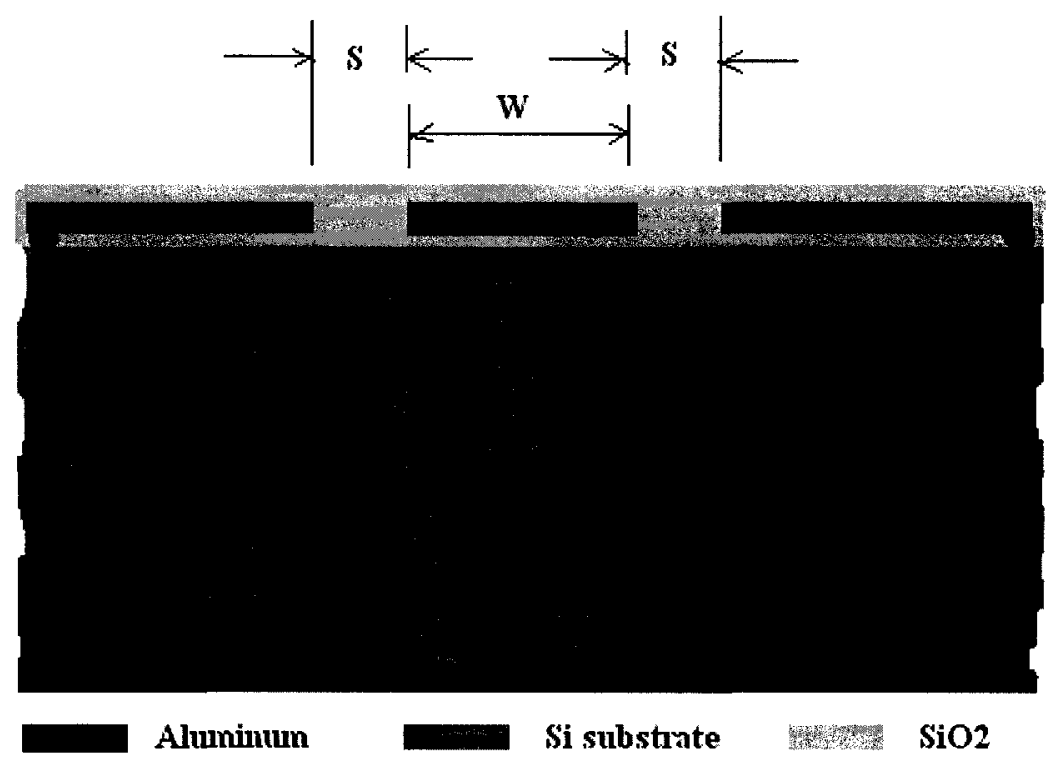

Figure 3-2 The cross-section view of a silicon CPW with pertinent dimensions.

Figure 3-2 gives a cross-sectional view of the silicon CPW [25]. Because a standard CMOS process is used, the center conductor (signal line of the CPW) is fully encapsulated in layers of dielectric glass, while the ground planes either make ohmic contacts to the underlying Si-substrate or can be left floating.

If the transverse CPW dimensions are much smaller than the shortest wavelength of the applied signals, a quasi-TEM mode can be assumed [25]. This means that the transverse fields carry the majority of the electromagnetic energy. In this study, the largest transverse dimension of the silicon CPWs is around $200 \mu \mathrm{m}$. The operation frequency is 5 to $50 \mathrm{GHz}$; that is, the shortest wavelength is above $3 \mathrm{~mm}$ since the 
relative effective permittivity is approximately 4 . Thus the quasi-TEM criterion is satisfied. At higher frequencies, higher order modes may be induced. The lowest mode above the TEM mode in the CPW line is the $\mathrm{TE}_{10}$ mode. Frankel and Hasnain gave more precise characterization of CPW lines without using the quasi-TEM assumption [39], [42]. The details are presented in Appendix A. The formulas in Appendix A may serve only as design guidelines for the implementation of silicon CPWs, since the circuit analyzed was much simpler than a real silicon CPW, the layer of dielectric material under the CPW metal not being included. To predict the behavior of a CPW on a silicon chip without using quasi-TEM assumptions would be very complicated; sophisticated mathematical mapping would be involved. It would be very hard to obtain explicit formulas. Fortunately, powerful numerical methods, such as FEM (Finite Element Method) as used in the HFSS software [50], and Method of Moments (MOM) in Sonnet [43], can help with simulations.

To analyze the CPW of Figure 3-2, the equivalent circuit needs to be defined. Two quasi-TEM equivalent circuits for silicon CPWs have been discussed in [25]. They are shown in Figure 3-3. The circuit in Figure 3-3(a) includes elements from the model of Shibata [29], which was derived from a full-wave computer analysis of a similar metal-insulator-semiconductor (MIS) structure. The circuit in Figure 3-3(b) is modified version of the model used by Zaghloul's group [25]. This group did intensive simulation and on-chip testing, and proved that quasi-TEM equivalent circuits can predict the measured silicon CPW parameters over a broad frequency range from 0.1 to $40 \mathrm{GHz}$, the circuit in Figure 3-3(b) is an improved model. In general, one can use 
the circuit of Figure 3-3 (c) as a theoretical model for silicon CPWs, where $Z$ is the total series impedance per unit length, and $Y$ is the total shunt admittance per unit length.

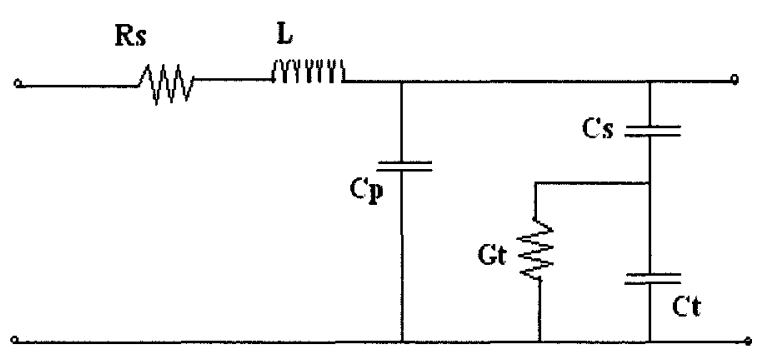

(a)

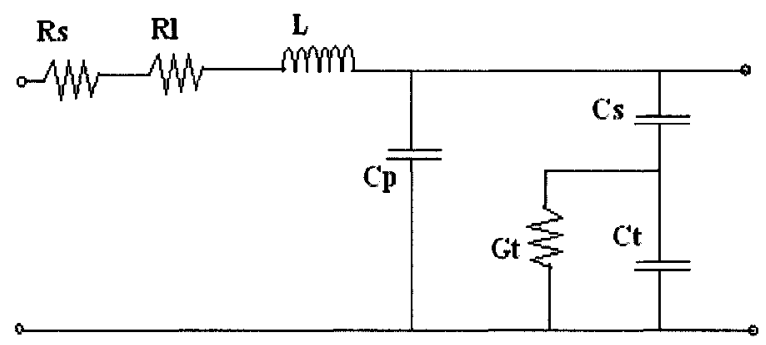

(b)

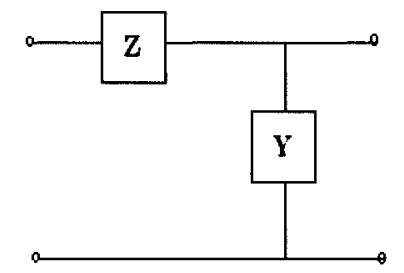

(c)

Figure 3-3 Quasi-TEM equivalent circuit of a silicon CPW: (a) Shibata's model, (b) modified model, (c) symbolic model.

In Figure 3-3, capacitor $C_{\mathrm{p}}$ represents the capacitance between the signal line of the CPW and the Si substrate. Capacitor $C_{\mathrm{s}}$ represents the capacitance between the signal line and the ground plane of the CPW. The conductance $G_{\mathrm{t}}$ is due to transverse current induced in the Si substrate by the electric field, while $C_{\mathrm{t}}$ similarly represents transverse displacement currents. Both $G_{\mathrm{t}}$ and $C_{\mathrm{t}}$ are determined by the electrical energy stored in the substrate. Elements $L$ and $R_{s}$ are the inductance and resistance of 
the aluminum conductors, respectively. $R_{l}$ represents the longitudinal current losses in the Si beneath the signal line.

In theory, the complex propagation constant $\gamma$ and the complex characteristic impedance $Z_{0}$ of CPW are determined from $Z$ and $Y$ as

$$
\gamma=\alpha+j \beta=\sqrt{Z \cdot Y} \quad \text { and } \quad Z_{0}=Z^{\prime}+j Z^{\prime \prime}=\sqrt{\frac{Z}{Y}}
$$

In practice, by measuring the S-parameters of the $\mathrm{CPW}$ lines, and using the $\mathrm{ABCD}$ cascade matrix, the propagation constant and characteristic impedance can be determined [38]. With the propagation constant and characteristic impedance known, the frequency-dependent equivalent-circuit parameters per unit length of the lines $(R$, $L, C$, and $G$ ) can be calculated from

$$
j \omega C+G=\frac{\gamma}{Z_{0}} \quad \text { and } \quad j \omega L+R=\gamma \cdot Z_{0} .
$$

Also, the attenuation factor $\alpha$ and the normalized phase factor $\beta c / \omega$ can be calculated from the complex propagation constant. Here, $c$ is the speed of light. The normalized phase factor also gives the effective permittivity, since $\beta c / \omega=\sqrt{\varepsilon_{r}}$. 


\subsection{Silicon CPW Losses}

There are three main contributions to the loss in silicon CPWs. The first is the conductor loss which includes metal skin-effect loss. The second is the silicon substrate loss, and the third is the radiation loss. The conductor loss is proportional to $f^{0.5}$ [37], the dielectric loss to $f$ [38], and the radiation loss to $f^{3}$ [39], where $f$ is the operating frequency.

Using a wider CPW signal line can reduce the conductor loss but at the expense of increasing the radiation loss [20]. Using a wider gap between the CPW signal line and the ground planes can also reduce the conductor loss but this will increase both substrate and radiation losses. For this research, silicon CPWs of various sizes have been simulated by using a commercial EM simulator ADS [44]. Figure 3-4 shows the attenuations of the CPWs with the signal line width $W=4 \mu \mathrm{m}$ and the gap between the signal and ground lines $S=4,8$ and $10 \mu \mathrm{m}$. Figure 3-5 shows the attenuations of the CPWs with the signal line width $W=8 \mu \mathrm{m}$ and the gap between the signal and ground lines $S=8,12,16$ and $24 \mu \mathrm{m}$. Figure 3-6 gives the attenuations of the CPWs with $W=10 \mu \mathrm{m}$ and $S=10,18,30$ and $40 \mu \mathrm{m}$. The simulation assumes a commercial CMOS $0.18-\mu \mathrm{m}$ process. The simulation parameters, such as the properties of the substrate and layout layers, were based on the data for the CMOS $0.18-\mu \mathrm{m}$ process. 
The simulation assumed that the top metal layer of the process (Metal 6) was used for the CPW lines.

The simulated results in Figure 3-6 are reasonable in view of the results in [25]. In this work, the attenuation values are almost 10 times lower than those in [25]. The reasons are as follows:

(1) In this study, a Si substrate with a resistivity of $10 \Omega \cdot \mathrm{cm}$ was used, whereas in [25], the substrate resistivity was in the $1 \Omega \cdot \mathrm{cm}$ range, which introduced much more substrate loss.

(2) In this work, the top metal layer of the process was used, while in [25], the second and lower layers were used. As the measured and simulated results in [25] indicate, the higher the metal layer is above the substrate, the lower the attenuation, since the substrate losses are the dominant CPW losses.

(3) Wide-gap (larger $S$ ) CPW structures were used in this study. In general this reduces the total losses. This point will be discussed in more detail in the next section.

The simulated results show that within a certain range, using wide $W$ and $S$ dimensions can reduce attenuation. Because the conductor loss is proportional to $f^{0.5}$, the dielectric loss to $f$, and the radiation loss to $f^{3}$, from the shapes of the attenuation 
curves we can see that, at frequencies below $50 \mathrm{GHz}$, the attenuation is due mainly to substrate and metal losses, and dominated by the substrate losses.

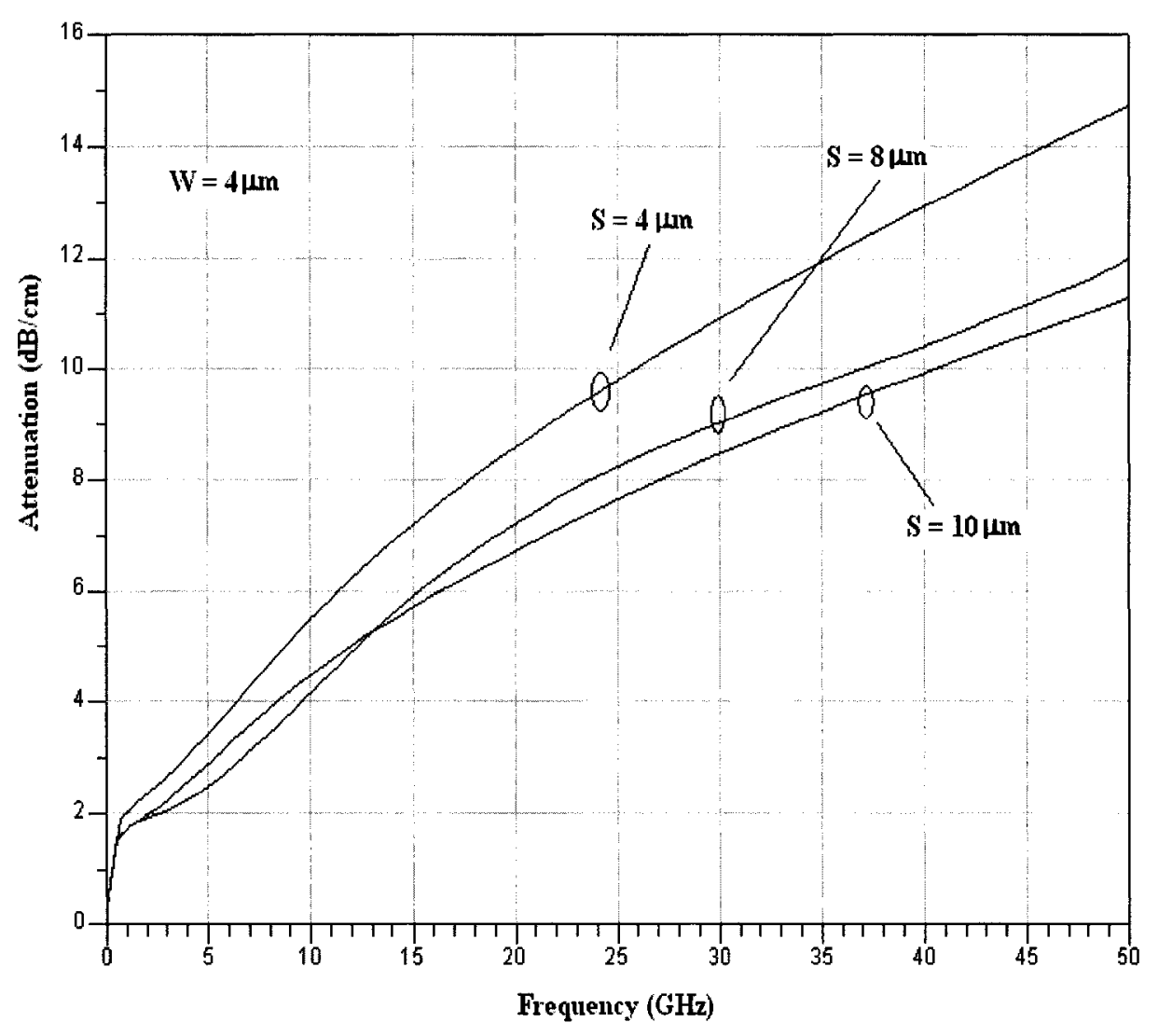

Figure 3-4 Attenuation of silicon CPWs ( $W=4 \mu \mathrm{m})$. 


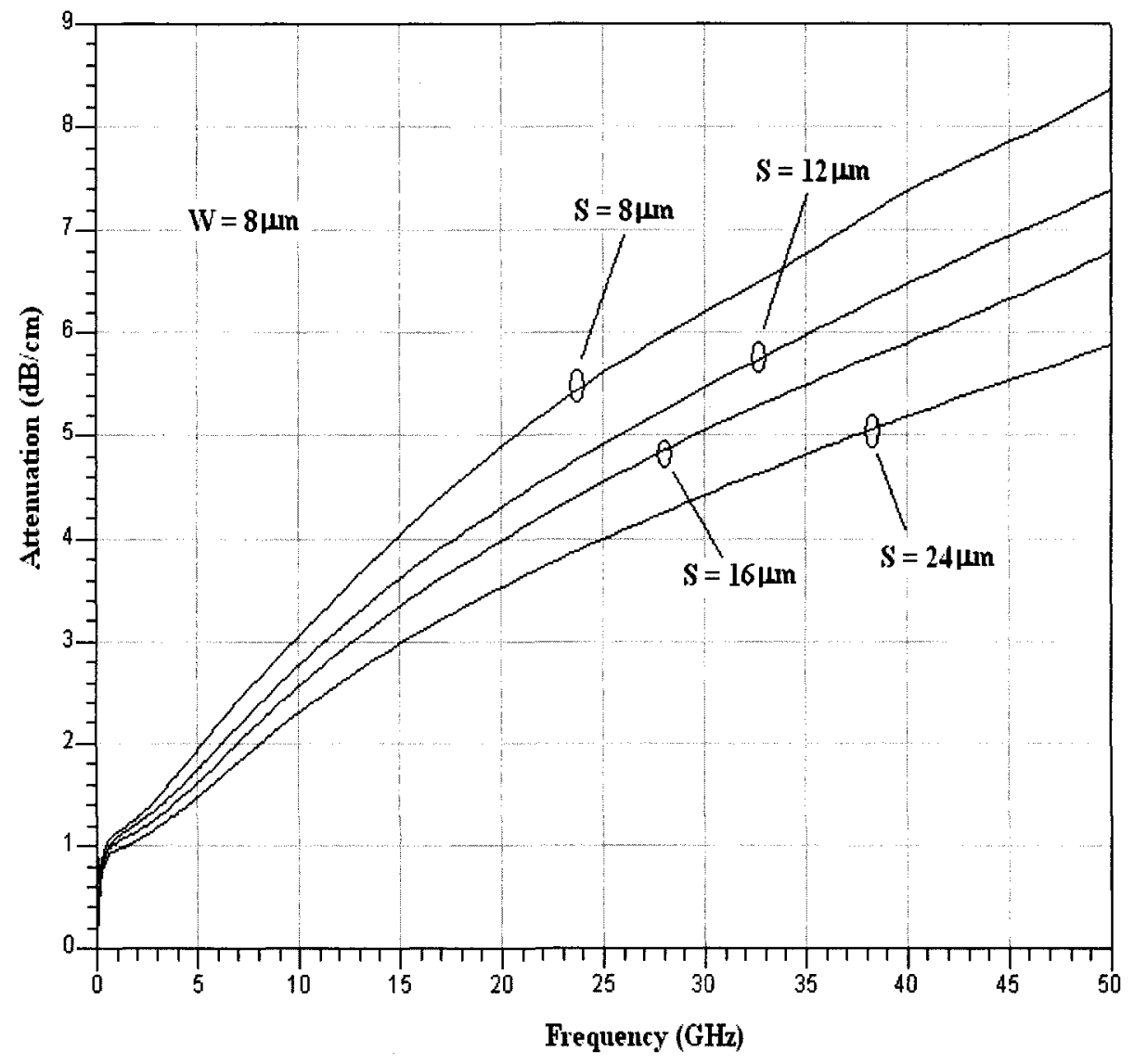

Figure 3-5 Attenuation of silicon CPWs $(W=8 \mu \mathrm{m})$. 


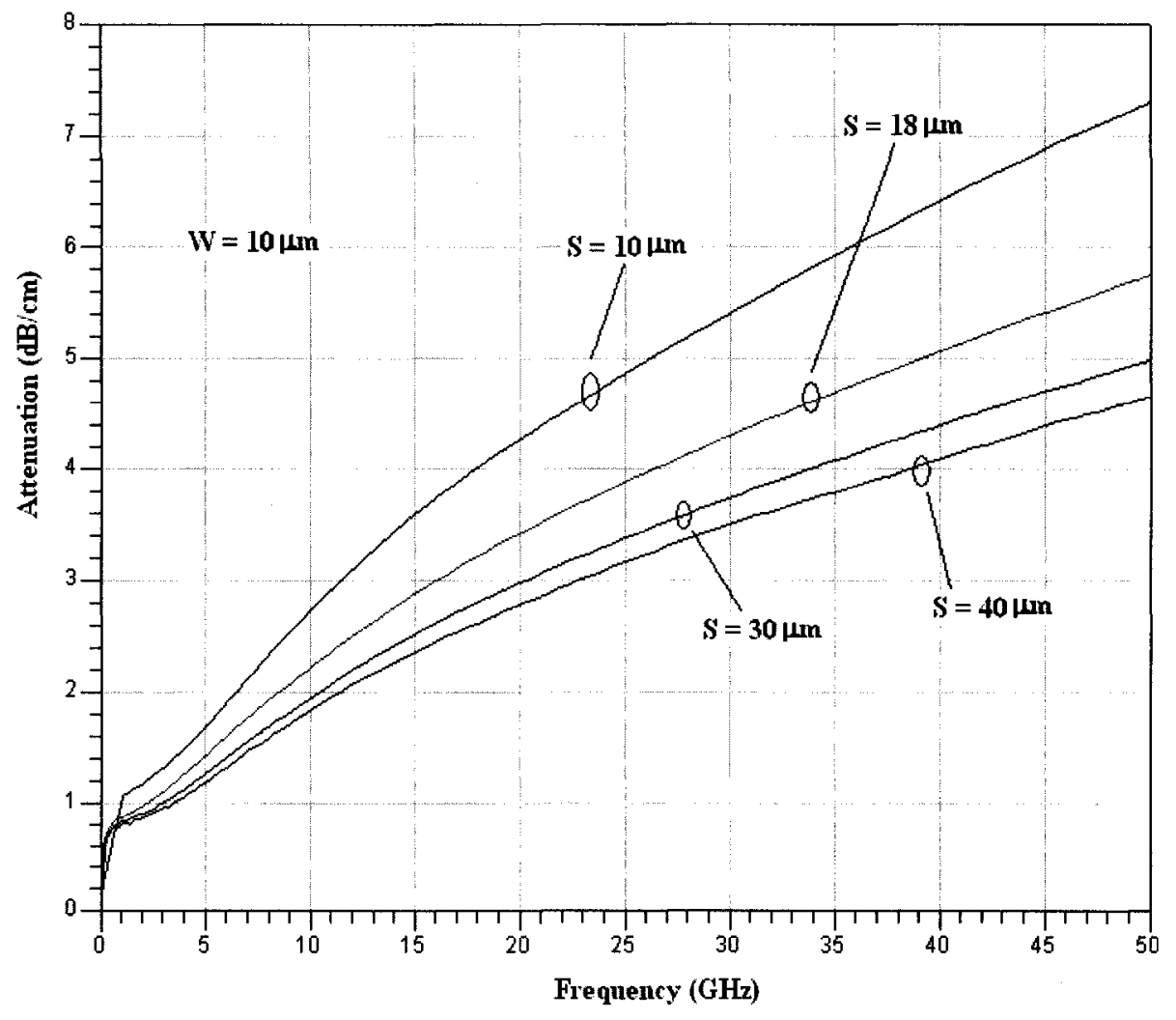

Figure 3-6 Attenuation of silicon CPWs $(W=10 \mu \mathrm{m})$.

\subsection{Slow-wave Technique for Substrate Loss Reduction}

CPWs on lossy silicon substrates tend to have high attenuations at frequencies above $20 \mathrm{GHz}$ [45], [25]. The loss is caused by parasitic coupling of energy from the CPW signal line to the lossy substrate. Using a CPW with a narrow gap between the signal and the ground lines can reduce the substrate loss but at the expense of 
increased conductor loss [20], [45]. Using a wide-gap line, on the other hand, reduces conductor loss but allows more electric field penetration into the silicon dioxide and the substrate, resulting in higher dielectric and substrate losses. Some research groups have suggested new design methodologies to overcome the problem. One of these methods was presented by Yook's group [46]. They introduced a so-called elevatedCPW. In this structure, the CPW signal line is elevated above the CPW ground planes and is further away from the substrate. This reduces the substrate losses. The other method, introduced by Harjani's group [47], uses multiple metal layers under the CPW signal line to shape the electric fields and prevent the energy from getting into the substrate. Yook's structure is difficult to fabricate on a standard CMOS chip since micromachining is required. Harjani's structure makes layout and fabrication much more complex.

A simpler approach is to add a ground shield underneath the silicon dioxide as suggested in [45]. This ground plane isolates the substrate from the signal line, reducing the substrate loss. However, since the return current flows mostly in the ground shield instead of the CPW ground, the skin effect loss is higher, which is due to microstrip-type behavior. Following Cheung and Long's idea [48], floating railwaytie-like metal strips, see Figure 3-7, are used as the ground shield instead of a solid metal plane, thereby improving the CPW performance. The advantages of this new structure, which is called slow-wave $\mathrm{CPW}$, or S-CPW, are that the ground shield 
return path is broken and the shield eddy currents are minimized, thereby reducing both skin effect and overall losses.

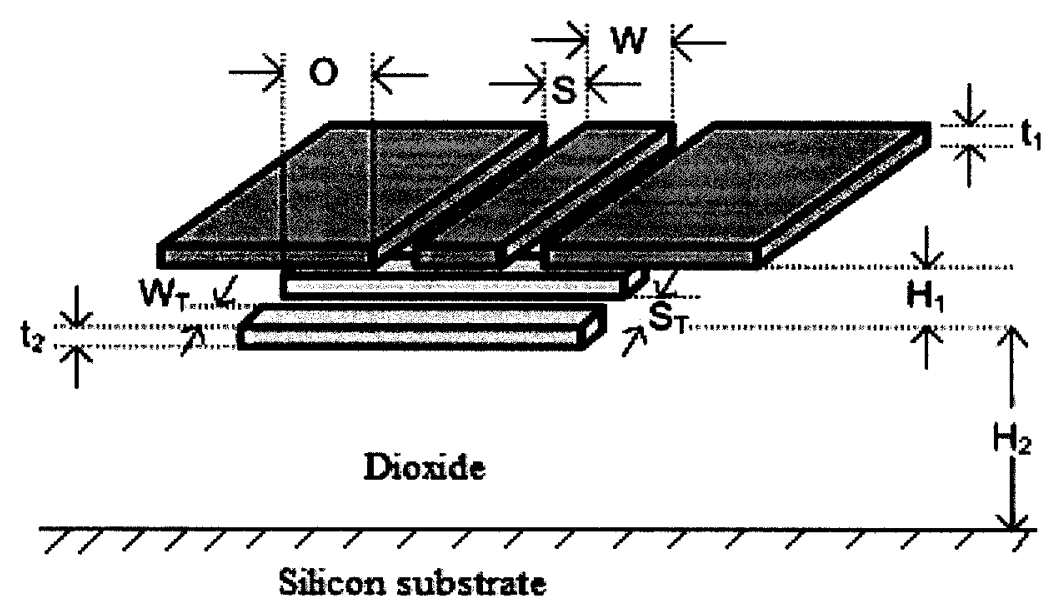

Figure 3-7 Slow-wave structure and design parameters.

The fundamental equations for the characteristic impedance $Z_{0}$ and phase velocity $V_{\mathrm{p}}$ of a lossless transmission line propagating a TEM mode are:

$$
Z_{0}=\sqrt{L / C} \text {, and } V_{p}=1 / \sqrt{L C} \text {. }
$$

Here, $L$ and $C$ are the unit-length inductance and capacitance of the CPW. Using floating ground strips allows the use of wide-gap CPW lines to reduce conductor 
losses, and also to achieve high characteristic impedance $Z_{0}$ (due to the large $L$ ). Since the floating ground strips are very close to the CPW layer, they increase $C$. Large $L$ and $C$ decrease the propagation velocity $V_{\mathrm{p}}$, so that the structure operates in a slowwave mode. Slow-wave propagation results in low loss, as observed by Hasegawa [26]. This loss reduction occurs in two ways. First, the silicon substrate loss is diminished because of the reduced $E$-field penetration therein. Second, the increased permittivity shortens the physical length of the CPW, further reducing the losses. Figure 3-8 shows the electromagnetic (EM) field distribution for both conventional and slow-wave CPWs.

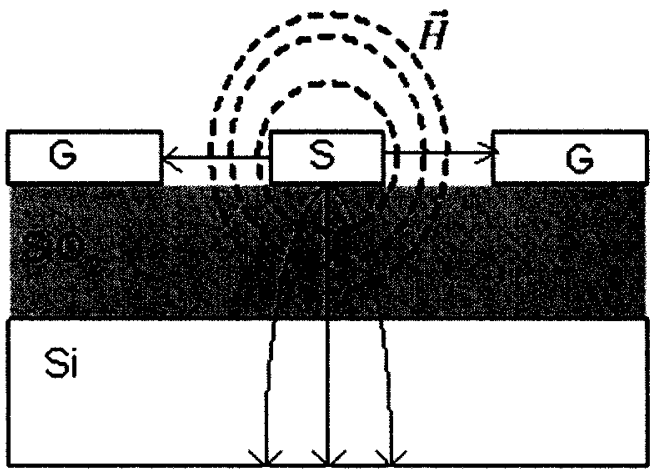

(a)

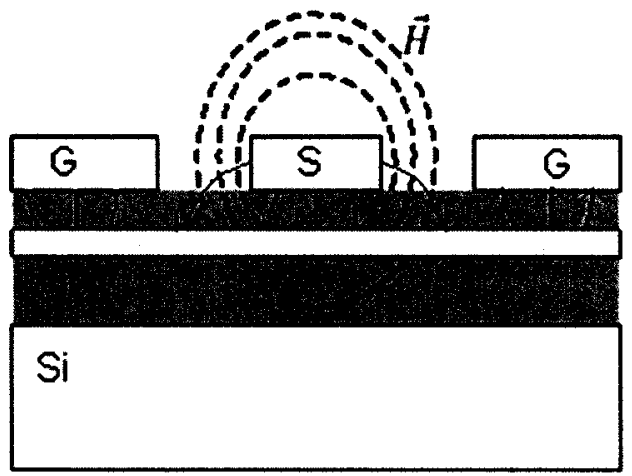

(b)

Figure 3-8 EM field distribution of (a) conventional CPW, (b) slow-wave CPW. 
Figure 3-8 shows that for a conventional CPW, most of the $E$-field flux goes into lossy silicon substrate, whereas for a slow-wave CPW, most of the $E$-field flux ends at the floating ground.

S-CPWs were first mentioned in 2004 [48], but attracted little attention since no design data were published. Furthermore, no one has built them on standard CMOS chips. To extend the results of [48], Amaya introduced new design parameters, such as $H_{1}, H_{2}, \mathrm{t}_{2}, W_{\mathrm{t}}, O$, and $S_{\mathrm{T}}$ as shown in Figure 3-7 [49]. These parameters were used by the author in fully three-dimensional EM modeling employing the HFSS software [50]. The dependence of the CPW characteristics on the geometry of the floating metal layer was intensively investigated, and the optimum dimensions were determined.

Slow-wave CPW techniques offer more design flexibility than is available with conventional CPWs. In conventional CPW the electrical parameters depend only on the centre conductor width $W$ and the gap $S$ between the centre conductor and the coplanar grounds, see Figure 3-7. S-CPW provides additional design parameters by introducing a floating electrode directly beneath the line's centre conductor. The vertical spacings $H_{1}, H_{2}$ for these floating electrodes and their thickness $t_{2}$ are limited and fixed for a given commercial process. Three extra design parameters for the SCPW are: the width $W_{T}$ of each floating electrode, the spacing $S_{T}$ between the floating electrodes, and the overhang projection $O$ of the floating electrode into the 
ground plane area, see Figure 3-7. This improved flexibility provides more freedom in the design, such as the tradeoff between wider center conductors with higher radiation loss and narrower ones with higher metallic loss, as well as the tradeoff between wide gaps with higher substrate attenuation and narrow ones with smaller impedances. Figures 3-9 and 3-10 present some simulation results. In these figures, the spatial "Duty Cycle" is $W_{\mathrm{T}} /\left(W_{\mathrm{T}}+S_{\mathrm{T}}\right)$.

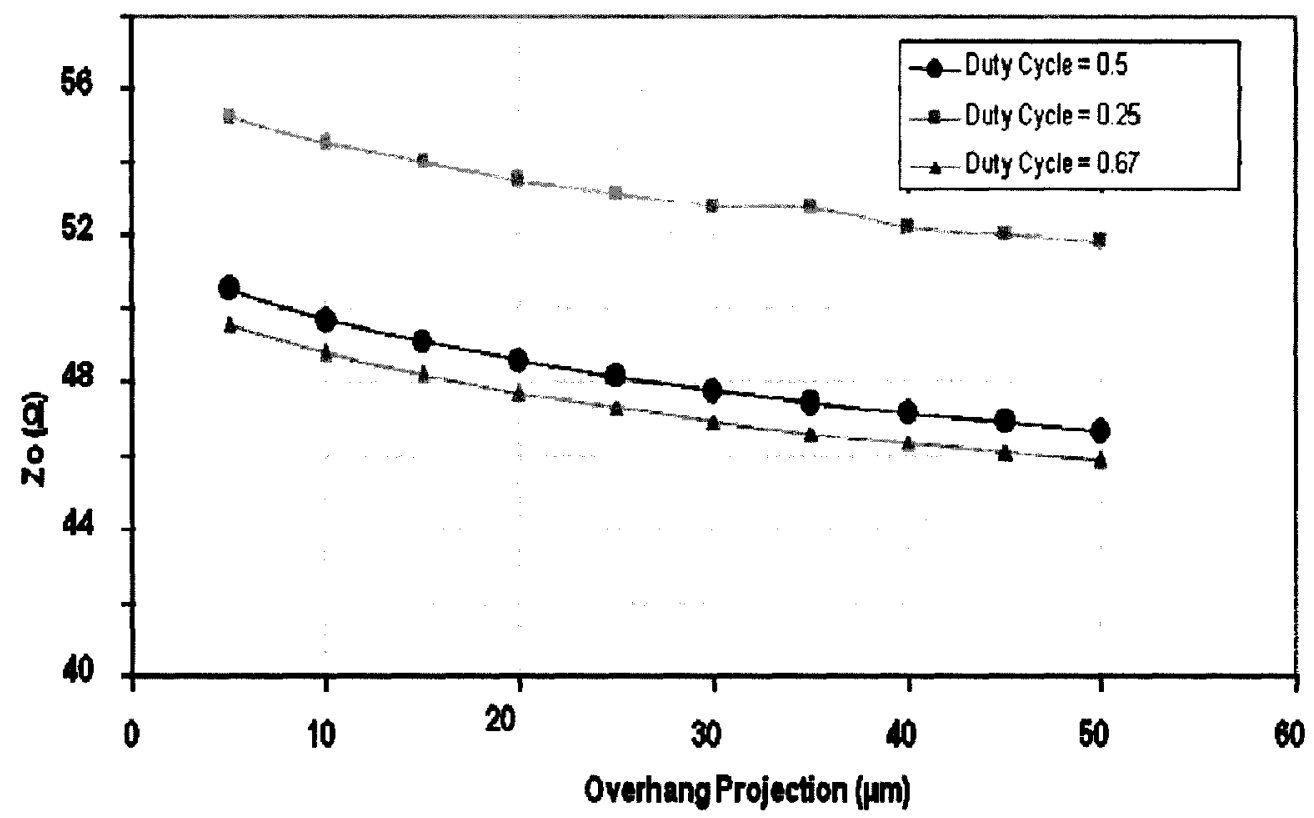

Figure 3-9 Effect of overhang projection $O$ and spatial duty cycle on line impedance for a slow-wave CPW. 


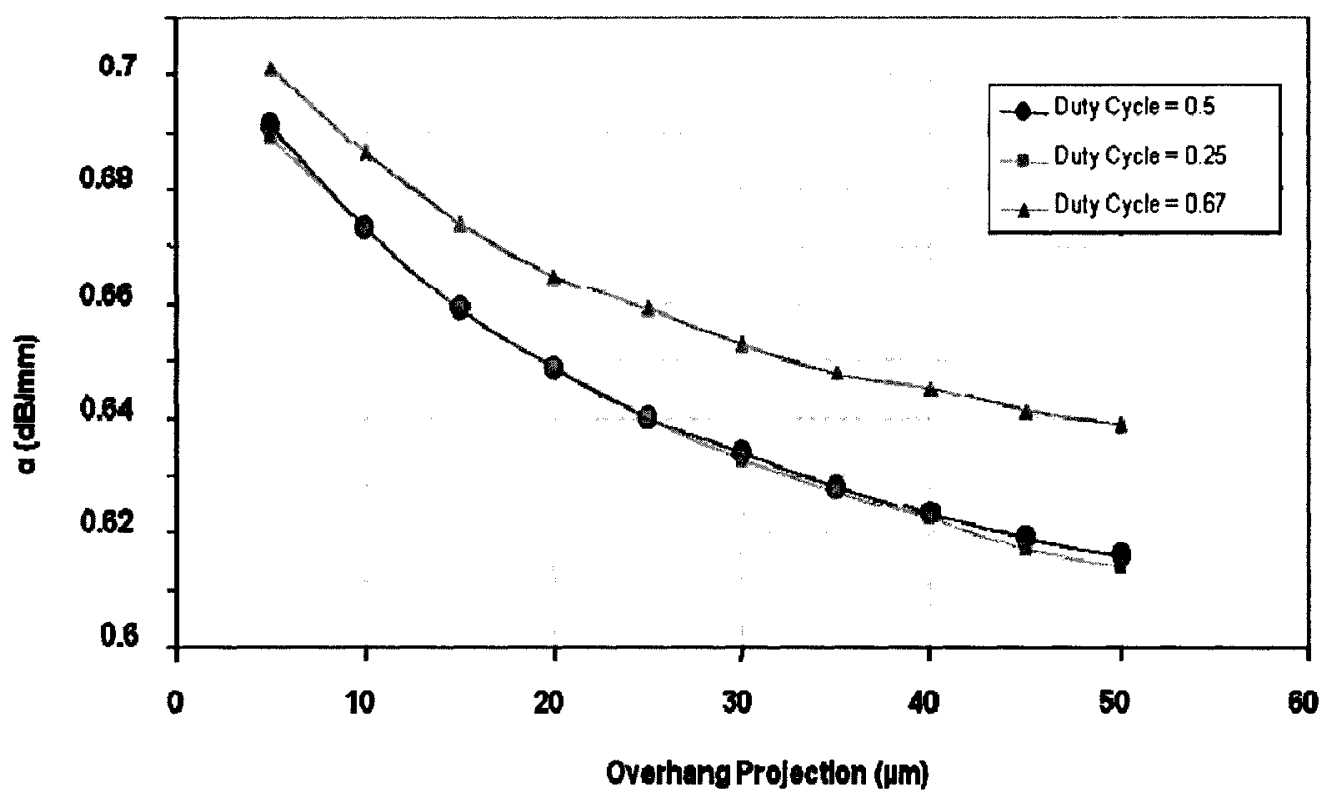

Figure 3-10 Effect of overhang projection $O$ and spatial duty cycle on line attenuation for a slowwave CPW.

Figures 3-9 and 3-10 show that

- By increasing the overhang $O$, the slow-wave CPW total capacitance increases, therefore decreasing the characteristic impedance $Z_{0}$ of the line.

- As the spatial duty cycle of the slow-wave CPW is increased from 0.25 to 0.5 and $0.67, Z_{0}$ decreases due to the added capacitance (the effective permittivity $\varepsilon_{\text {eff }}$ increases). 
- The slow-wave CPW attenuation decreases as the spatial duty cycle is increased beyond 0.5 , because the $E$ fields are confined near the surface, reducing dielectric and radiation losses.

Figure 3-11 shows the HFSS setup for simulating a slow-wave CPW.

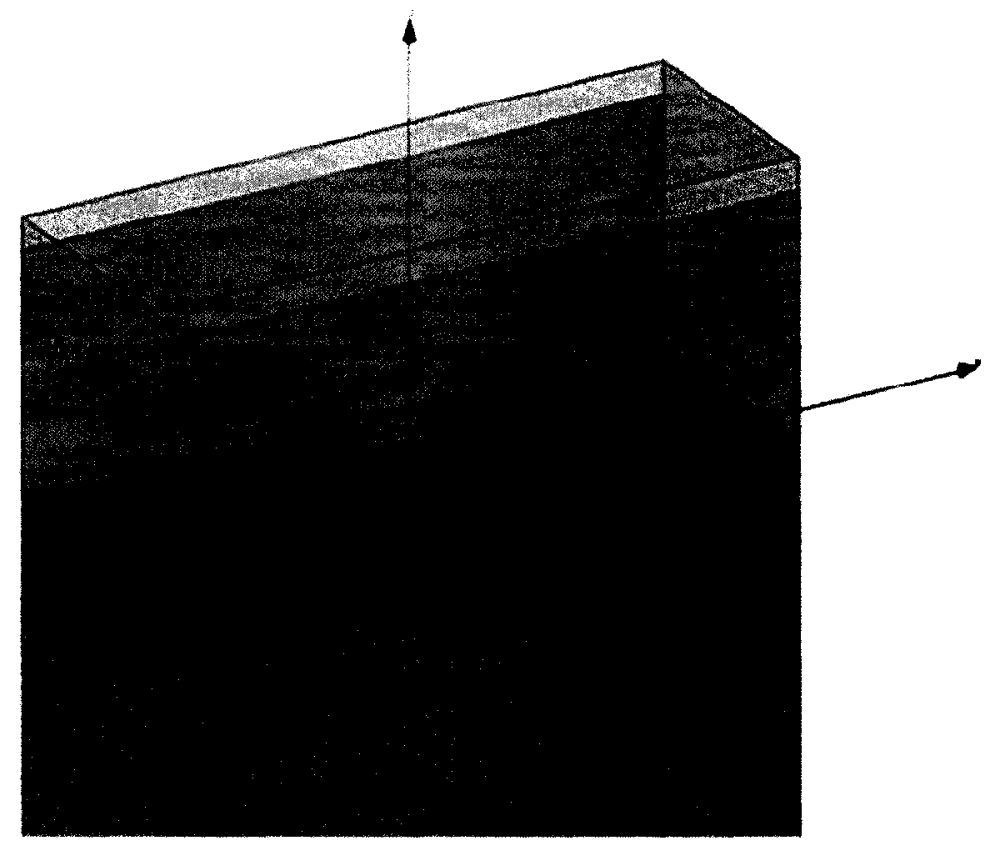

Figure 3-11 HFSS geometry for a slow-wave CPW simulation. M5 and M6 denote process metal layers.

In this figure $L$ is the length of the transmission line section under simulation. $L$ can be set to a much smaller value than the real length by using 'Wave Port' in HFSS, since the simulator assumes that the same structure extends, along the propagation direction, 
beyond the simulation area. Using a small $L$ can save the simulation time. The results are then normalized with the real lengths. Here $L$ is set to $50 \mu \mathrm{m}$.

As a comparison, the electric field distributions for both conventional and slowwave CPWs are shown in Figures 3-12 and 3-13, which were produced by using HFSS simulator [50]. It is evident that the slow-wave CPW with added floating ground confines the $E$-field to the neighborhood of the signal line and therefore reduces the substrate loss.

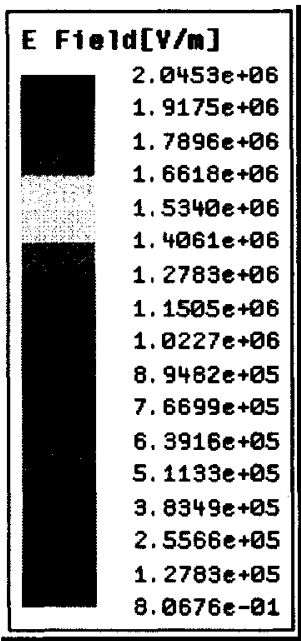

Figure 3-12 $E$-field distribution of a conventional CPW. 


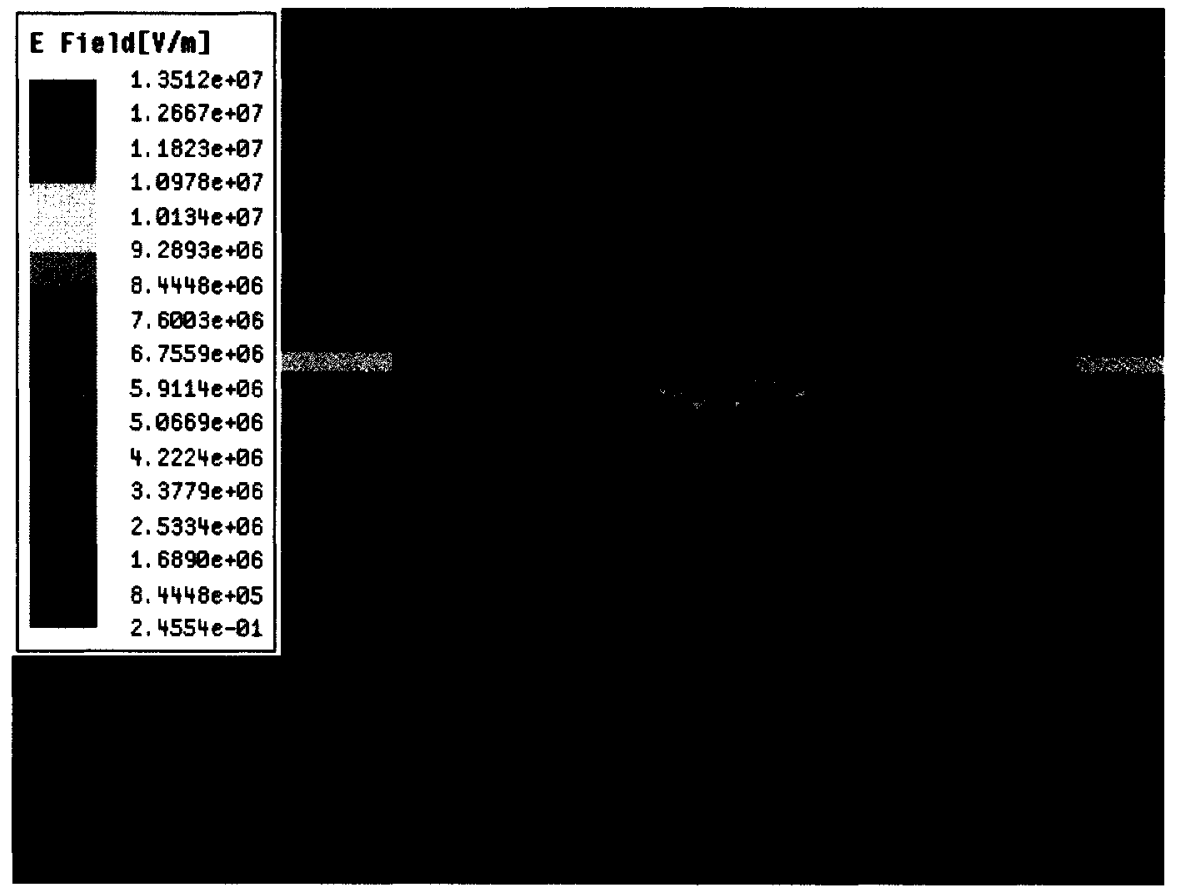

Figure 3-13 E-field distribution of a slow-wave CPW.

\subsection{Silicon CPW Implementation}




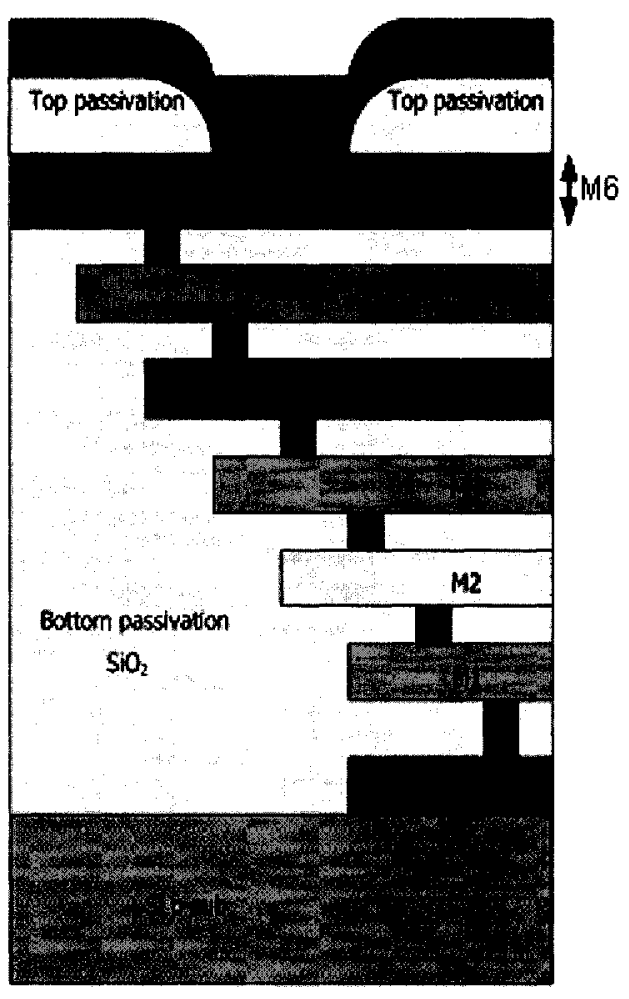

Figure 3-14 Technology stack for the CMOS 0.18- $\mu \mathrm{m}$ process.

After simulation and optimization, it was possible to realize a few S-CPWs in a CMOS $0.18-\mu \mathrm{m}$ process. The process used for this research provides six metal layers, as shown in Figure 3-14. The top layer (M6) is the thickest and is the furthest from the substrate. The CPW was fabricated in this layer to minimize metallic and substrate losses. The floating ground strips were built in the second layer (M5), which is much thinner than the top layer, but thicker than the other four lower layers. Another benefit of making the second layer a ground-shield layer is that it is spaced above the substrate. Even if a small amount of electric field energy leaks through the metal strips, it remains mostly in the much less lossy silicon dioxide region. 
Figure $3-15$ is a photograph of the $2.0 \times 1.5 \mathrm{~mm}^{2}$ chip. The second row and the first half of the third row contain the slow-wave CPW and conventional CPW components. The on-chip $S_{21}$ measurements are very promising. Figure 3-16 shows the measured and simulated results for a slow-wave CPW and a conventional CPW. Here a $10-\mu \mathrm{m}$-wide signal line and a $30-\mu \mathrm{m}$-wide gap are used for both types of CPWs. Both are $200 \mu \mathrm{m}$ long. For the floating ground of the slow-wave CPW, the strip width is $5 \mu \mathrm{m}$, and the gap is $0.5 \mu \mathrm{m}$. Figure 3-16 shows that the slow-wave CPW has much lower insertion loss than the conventional CPW, especially at higher frequencies. Notice that, with the same size of CPW, adding the floating ground strips increases $C$ and decreases $Z_{0}$. The measured $Z_{0}$ of the conventional CPW is $\sim 85 \Omega$, while it is $\sim 50$ $\Omega$ for the slow-wave CPW. The conventional CPW has more return loss than that of the S-CPW, which is near zero. Allowing a 20-30\% return loss deduction, the overall loss of the conventional $\mathrm{CPW}$ is still greater than that of the slow-wave $\mathrm{CPW}$, and increases with frequency more rapidly. Having a very low return loss, the overall loss of the slow-wave CPW line is approximately equal to $S_{21}$, which is $\sim 0.25 \mathrm{~dB} / \mathrm{mm}$ at 40 $\mathrm{GHz}$.

Figure 3-17 shows the measured effective relative permittivity $\varepsilon_{r, e f f}$ results for the slow-wave $\mathrm{CPW}$ and the conventional $\mathrm{CPW}$ lines. One can see from this figure that by adding the floating ground, the $\varepsilon_{r, e f f}$ is increased by $\sim 2.5$ times. This means that the propagation velocity and wave length are decreased by a factor of $\sim 1.5$. 


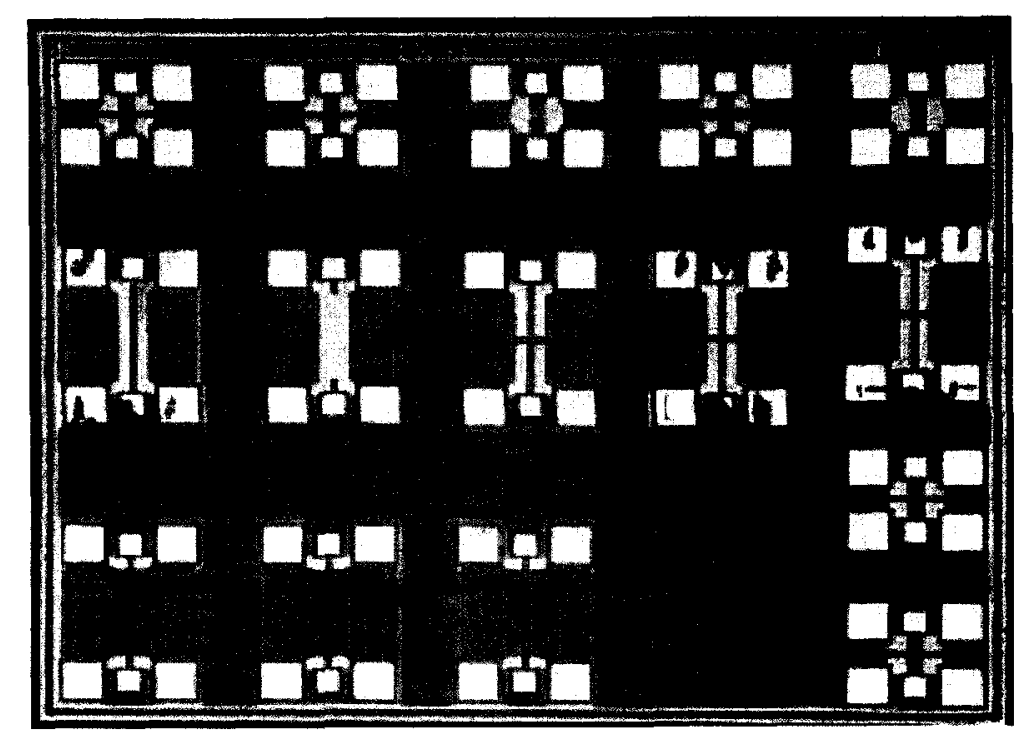

Figure 3-15 CMOS 2.0 $\times 1.5 \mathrm{~mm}^{2}$ chip with CPW, S-CPW and NMOS varactor components.

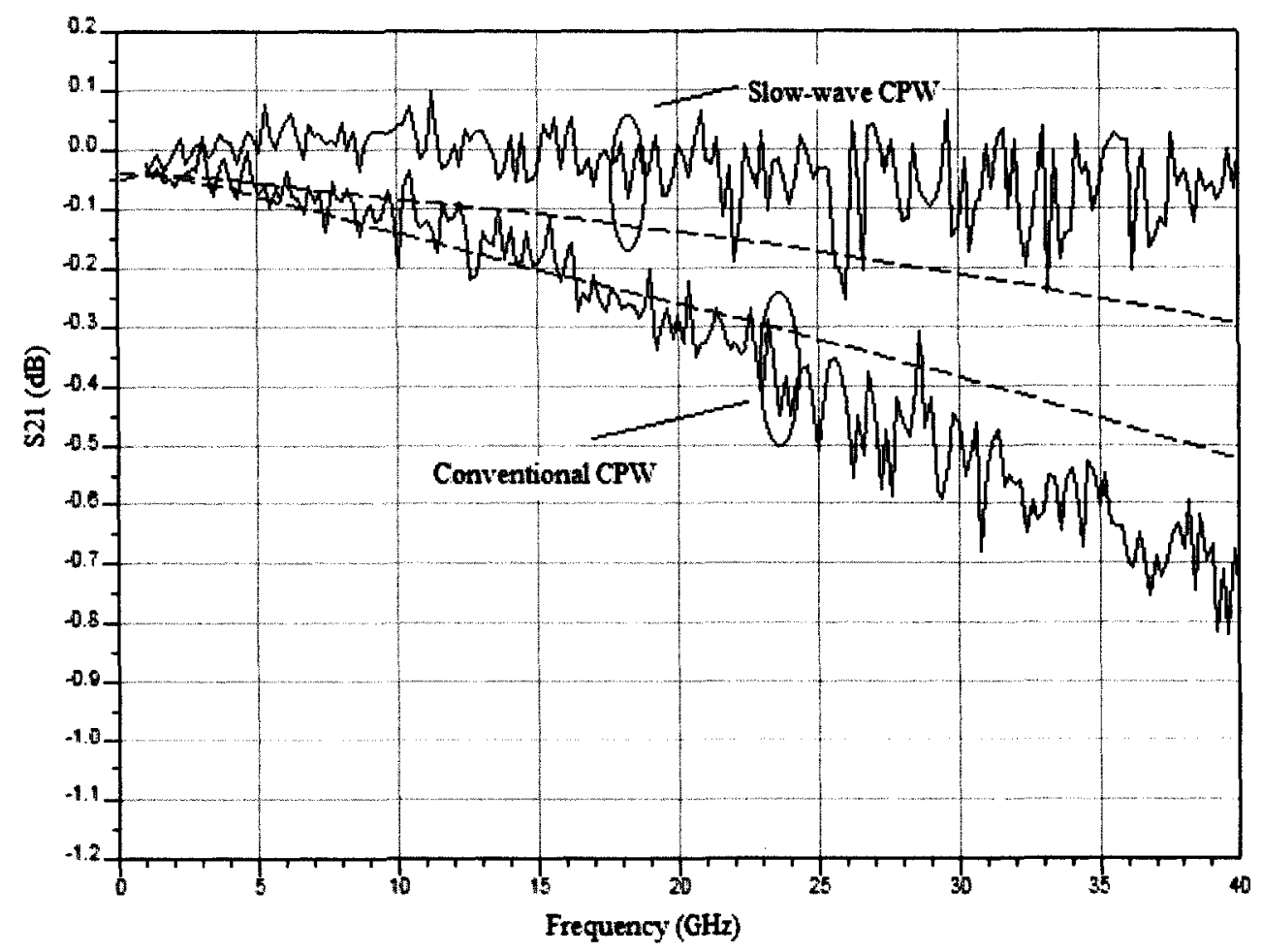

Figure 3-16 Comparison between the measured ( - ) and simulated $(-)\left|S_{21}\right|$ for a conventional and a slow-wave CPW. 


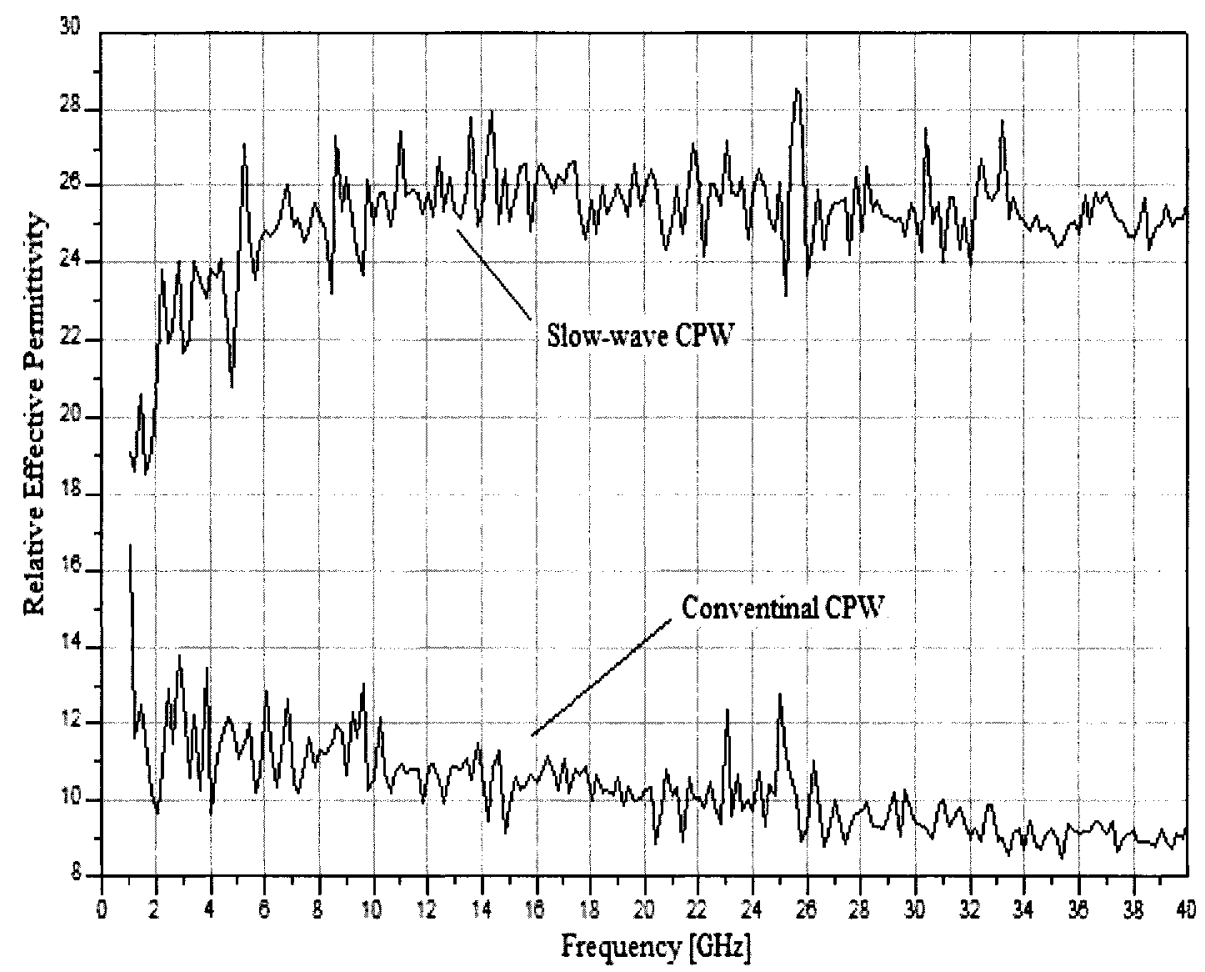

Figure 3-17 Effective relative-permittivity $\varepsilon_{r, e f f}$ for a conventional and a slow-wave CPW.

The CPW S-parameters were measured with an HP 8722ES vector network analyzer (VAN) at frequencies up to $40 \mathrm{GHz}$ using Picoprobe ground-signal-ground (GSG) probes. The short-open-load-through (SOLT) calibration and de-embedding technique was used to subtract out the pad parasitics [67]. 


\subsection{Conclusion}

This chapter mainly concerns the performance of silicon CPWs. One big drawback is their high substrate losses. The lower the substrate resistivity, the larger the loss. The low resistivity of the $10-\Omega \cdot \mathrm{cm}$ commercial substrate used here requires design efforts to overcome the loss. A new technique, slow-wave CPW, was introduced in this chapter, and the extensive simulation, chip design and chip measurement procedures are described. This research results show that using slowwave structures in all-silicon CPW design is a very good solution, especially for high frequency operations. 


\section{Chapter 4 CMOS Varactor Characterization}

MOS varactors are key components in all-silicon NLTLs. The nonlinear $C(V)$ characteristics of the varactors, together with the transmission line parameters, determine the nature of the nonlinear wave propagation along the NLTL. Varactors used in RF applications such as voltage controlled oscillators need a high quality factor $Q$ and a capacitance-voltage characteristic $C(V)$ that provides a large linear tuning range. In contrast, varactors used in pulse-compression nonlinear transmission lines (NLTLs) require a large cutoff frequency $f_{c}$ for high-order harmonic generation, and a $C(V)$ characteristic specifically optimized for a high degree of nonlinearity. Specific monotonic or non-monotonic $C(V)$ characteristics can be optimized for either single-edged or double-edged pulse compression.

\subsection{CMOS Varactor structures}

In order to choose the most suitable varactors for use in NLTL pulse compression, six different CMOS transistor-based varactor structures (Figure 4-1) were investigated in this study. 


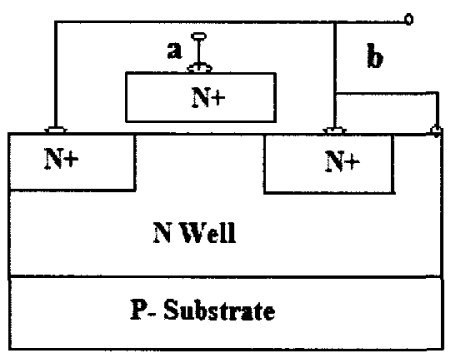

(1)

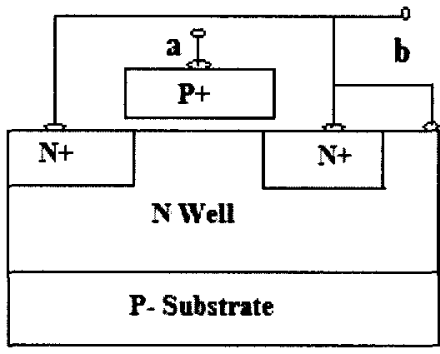

(2)

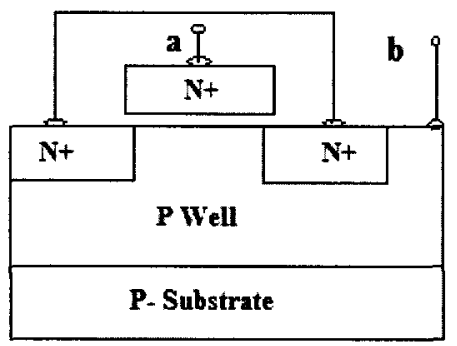

(3)

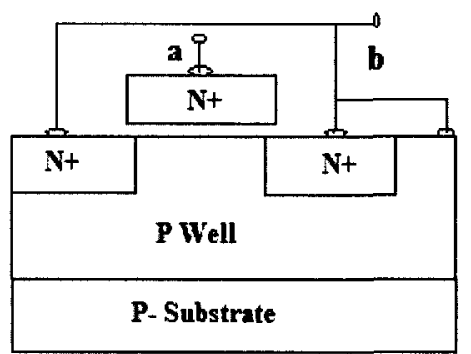

(4)

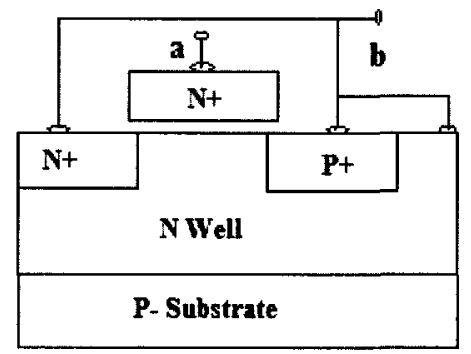

(5)

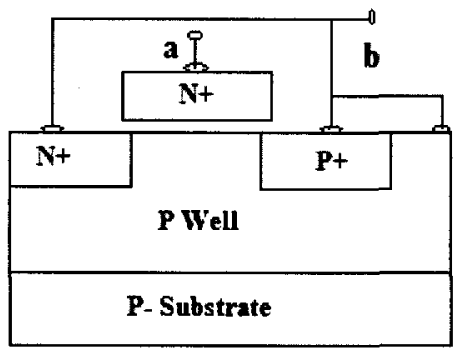

(6)

Figure 4-1 Cross-sections of six varactor structures.

In Figure 4-1, each varactor has two terminals: one connects to the gate (terminal ' $a$ '), the other to the bulk (terminal ' $b$ '). The source and the drain are interconnected, and are either connected to the bulk (structures 1, 2, 4, 5, and 6) or left floating (structure 3). Structure 1 has an $N$-type gate, with the $N$-type source and drain formed in an $N$-type well, creating an accumulation-mode MOS (AMOS) varactor with a monotonic $C(V)$ characteristic [51]. Structure 2 is slightly different: its use of a $P$-type 
rather than an $N$-type gate shifts the transistor threshold voltage and displaces the $C(V)$ curve by $\sim 1 \mathrm{~V}$ along the voltage axis. Structure 3 is a standard NMOS varactor, with its source-drain floating relative to the bulk, making the device work in inversion mode (IMOS). It too has a monotonic $C(V)$ curve. Structure 4 is also a standard NMOS varactor but the source-drain is connected to the bulk, making the device work in all three modes -inversion, depletion and accumulation- producing a nonmonotonic $C(V)$. Structure 5 has differently-doped source and drain; one is $N$-type, the other $P$-type. We use this structure to supply minority carriers to an AMOS device (structure 1), enabling it to work not only in accumulation but also in depletion and inversion modes. Its $C(V)$ is non-monotonic. Structure 6 is similar to structure 5 , but it supplies minority carriers to an IMOS device (structure 3). Structure 6 also has a nonmonotonic $C(V)$. Since it has an $N$-source and a $P$-drain, it is referred to here as a SnDp device.

\subsection{C(V) Characteristics and Cutoff Frequency}

To evaluate the advantages and disadvantages of each structure, a commercial device simulator, Synopsis Medici [52], was used. The $Y$-parameters of the six MOS varactor structures were generated at $0.5 \mathrm{GHz}$ using the $\mathrm{DC}$ and $\mathrm{AC}$ small-signal analysis features of the simulator. Here, $Y=G+j \omega C$, since Medici assumes a simplified varactor model consisting of a capacitance in parallel with a conductance. Figure 4-2 presents an extracted model of a varactor. The parasitic $L$ 's and $R^{\prime}$ 's can be 
removed from all three terminals - DS (drain and source), G (gate) and B (bulk) - by using Koolen's technique [68]. This yields the intrinsic model (shown in the brokenline box in Figure 4-2), for which the input admittance $Y_{\text {in }}$ looking into terminal $G$ can be extracted as

$$
Y_{\text {in }}=j \omega\left[\frac{C_{o x} \cdot C_{d e p}}{C_{o x}+C_{d e p}}+C_{\text {parasitic }}\right]+G_{d e p}+G_{\text {paeasitic }}
$$

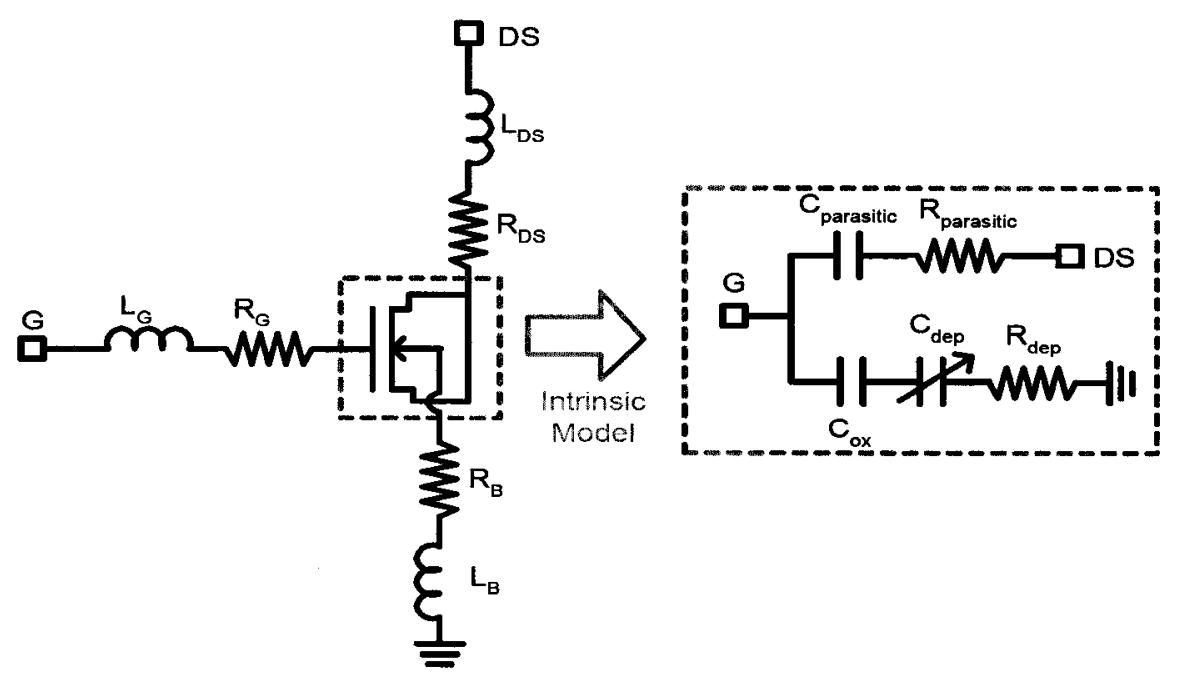

Figure 4-2 CMOS intrinsic varactor model.

In the simulations, each unit-cell varactor structure was given a gate length of 0.5 $\mu \mathrm{m}$, a gate width of $1.0 \mu \mathrm{m}$, a gate oxide thickness of $0.025 \mu \mathrm{m}$, a well doping level of $6 \times 10^{17} / \mathrm{cm}^{3}$, a terminal diffusion doping level of $3 \times 10^{20} / \mathrm{cm}^{3}$ and a substrate doping level of $10^{15} / \mathrm{cm}^{3}$. The explanations for these simulation settings are: (1) The longer 
the gate length, the bigger the $C_{\max } / C_{\min }$ ratio, but the lower the cutoff frequency [54]. It is found in this study that, for NLTL pulse-compression applications, $0.5-\mu \mathrm{m}$ gate length can give compromised values for both $C_{\max } / C_{\min }$ ratio and cutoff frequency. (2) Due to its 2-D feature, Medici assumes 1.0- $\mu \mathrm{m}$ gate width for all the devices under the simulation. To obtain the real results, the simulated results need to be characterized by the gate width. (3) The gate oxide thickness and doping level used here are "roadmap" values, they are typical of today's CMOS technologies [53].

Figure 4-3 presents the $C(V)$ curves of the six structures. As expected, structures 1,2 , and 3 have capacitances that vary monotonically with bias voltage, while structures 4,5 , and 6 have non-monotonic $C(V)$ s. In the first group (Figure 4-3, top), structure 3 (IMOS varactor) shows a slightly higher $C_{\max } / C_{\min }$ ratio (>5) than the other two. The $C(V)$ of structure 3 is very nonlinear with a sharp $C_{\max }$ to $C_{\min }$ transition, favouring high harmonic generation in pulse compression. In the second group (Figure 4-2, bottom), the three structures have similar $C_{\max } / C_{\min }$ ratios. The differences are in their threshold voltages, and therefore in the voltage ranges of their nonlinear $C(V) \mathrm{s}$. For structures 5 and 6 this range is from -3.5 to $+0.5 \mathrm{~V}$; for the others it is from -2.0 to $+2.0 \mathrm{~V}$. 

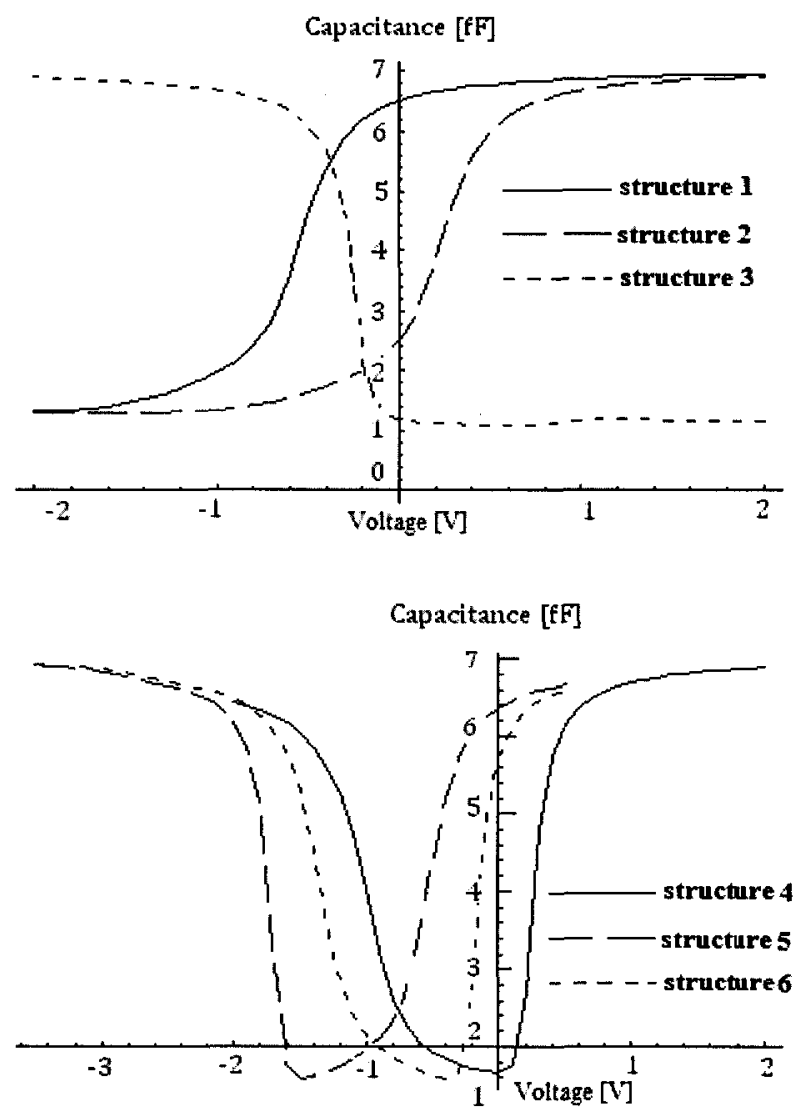

Figure 4-3 $C(V)$ characteristics of the six CMOS varactor structures.

Figure 4-3 also shows that the $C(V)$ curve of IMOS (structure 3 ) is similar to that of MOS capacitors. This occurs because the IMOS structure is similar to a MOS capacitor structure. But IMOS varactors have higher $C_{\max } / C_{\min }$ ratios. Also the switching speed of IMOS varactors is much faster than that of MOS capacitors. The IMOS varactor acts as a gate-controlled diode, as Grove pointed out [54]. In the IMOS varactor, at inversion mode, minority carriers can be supplied by an external circuit to the inversion layer through a side of the diffusion region, enabling the gate 
capacitance to increase rapidly to the value of the oxide capacitance. But in a pure MOS capacitor, minority carriers must be generated thermally in the bulk, which is an extremely slow process. This explains the difference between IMOS varactors and MOS capacitors [54].

By equating the varactor's calculated admittance $Y=G+j \omega C_{p}(v)$ with the reciprocal of its series-model impedance $R_{v}(v)+1 /\left[j \omega C_{v}(v)\right]$, the (bias-dependent) cutoff frequency is found to be

$$
f_{c}=\frac{1}{2 \pi R_{v} C_{v}(v)}=\frac{\omega^{2} C_{p}(v)}{2 \pi G}(\mathrm{~Hz})
$$

Figure 4-4 gives the $f_{c}(V)$ curves of the six structures. In the upper group of Fig. 44 , structure 3 has the highest maximum $f_{c}$ but the lowest minimum $f_{c}$. Structures 2 and 3 have a slightly higher large-signal cutoff frequency $(\sim 690 \mathrm{GHz})$ than that of structure $1(\sim 620 \mathrm{GHz})$. Here, large-signal cutoff frequency, $f_{c, l s}$, is calculated by taking the average $f_{c}$ value over the gate voltage range. Higher $f_{c}$ also means a higher $Q$ factor, since $Q=\omega C / G$. In the second group of Figure 4-3, structure 5 has the highest maximum $f_{c}$ while structures 5 and 6 both have lower minimum $f_{c}$ than structure 4 since the bottom plate of the capacitor is formed by the inversion-layer, increasing the resistance [55]. Structure 5 has the highest average $f_{c}$ and structure 6 has the lowest average $f_{c}$. In general, when the device resistance is relatively high, that is, when the oxide-silicon interface is depleted, $f_{c}$ is high. The value of $f_{c}$ also depends 
on the individual varactor structures. This is because of the bias-dependence of the varactor resistance $R_{v}$. In practice, setting the bias voltage slightly below the threshold value $V_{\mathrm{t}}$ (around $0.7 \mathrm{~V}$ for the CMOS process) will keep the $f_{c}$ high.

Structure 3 (IMOS) from the first group is chosen as the monotonic-type varactor for single-edge compression because of its highly nonlinear $C(V)$, its compatible $f_{c}$ and its availability in standard CMOS processes. In some applications the AMOS varactor would be a better candidate because of its higher average $f_{c, l s}$. Structure 4 from the second group is chosen as the non-monotonic-type varactor for double-edge compression, due to its standard-CMOS-process availability, even through structure 5 might be a better candidate. A further reason for choosing NMOS-type varactors for both structures is that their majority carriers are electrons, whose mobility is nearly twice that of holes. These devices are good for high frequency operation. Implementation and measured results for the two NMOS varactors are presented in the next section. 


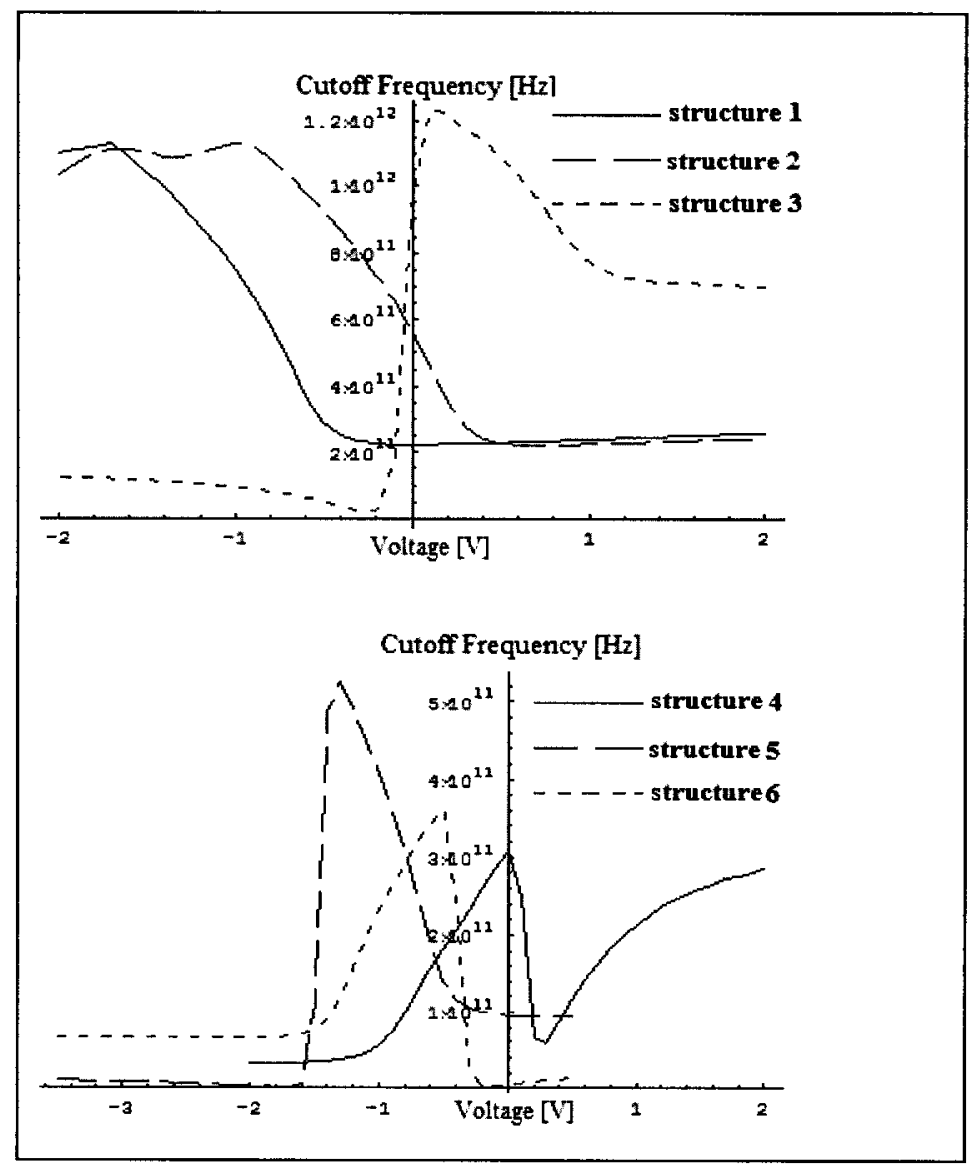

Figure 4-4 $f_{c}(V)$ characteristics of the six CMOS varactor structures.

\subsection{NMOS Varactor Implementation}

Structure 3 is named an "SDF" varactor since its source and drain float independently from the bulk, and structure 4 is called an "SDB" varactor since its 
source and drain are connected to the bulk. Both varactors were fabricated in a standard $0.18-\mu \mathrm{m}$ CMOS process.
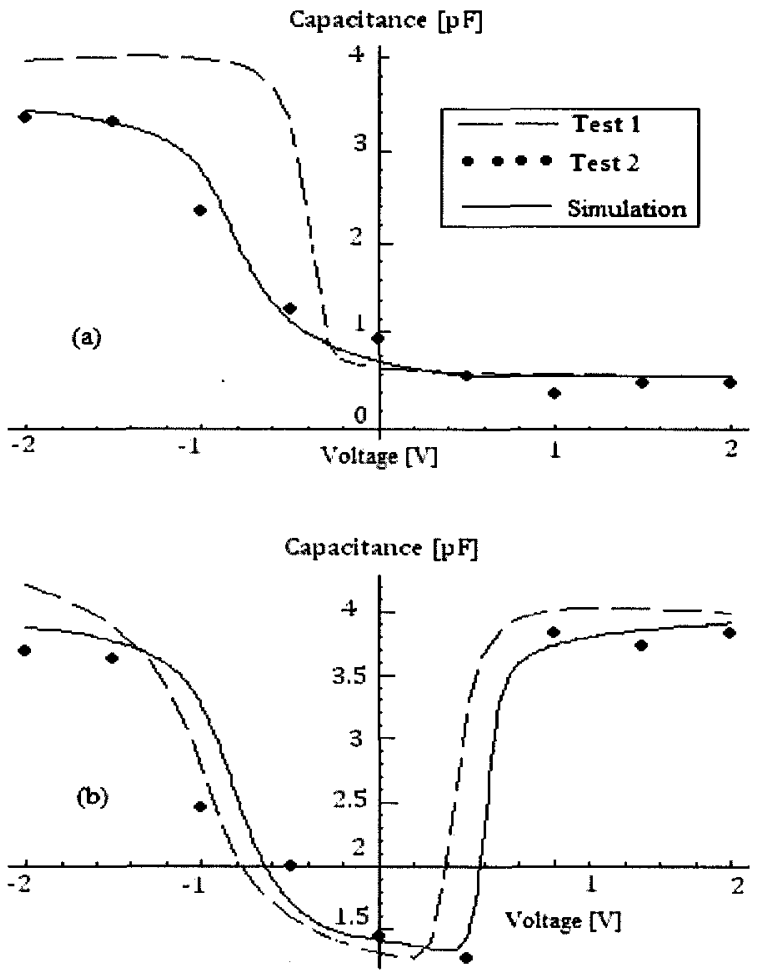

Figure 4-5 Measured and simulated $C(V)$ curves of (a) an SDF varactor and (b) an SDB varactor.

Both types of varactors were built on a CMOS chip (top row in Figure 3-15). Each varactor has a gate of length of $1.0 \mu \mathrm{m}$ and width $7.5 \mu \mathrm{m}$, and a total of 60 fingers. In Figure 4-5, the 'Test1' curves were obtained with an HP 4280A 1-MHz capacitance meter. On-chip pad parasitics were not subtracted. The 'Test2' curves were obtained using an HP 8722ES vector network analyzer, two ground-signal-ground (GSG) probes, and two Picosecond 5541A 26-GHz bias tees. In this case, the pad parasitics 
were de-embedded and the data were derived from network parameters at $10 \mathrm{GHz}$. The 'Simulation' curves were obtained from ADS simulations, using the varactor models, also at $10 \mathrm{GHz}$. The difference between curves 'Test1' and 'Test2' in the vicinity of $-2 \mathrm{~V}$ is due to pad parasitic effects.

Taking the chip intrinsic parameters into account, it is found that a longer gate length provides a larger $C_{\max } / C_{\min }$ ratio, but at the expense of increased varactor losses and decreased $f_{c}$. For the large varactors in Figure 4-5, the measured average $f_{c, l s}$ is $\sim 190 \mathrm{GHz}$ for the SDF type and $\sim 120 \mathrm{GHz}$ for the SDB type. The measured $C_{\max } / C_{\min }$ is $\sim 6$ for the SDF type and $\sim 3$ for the SDB type. In these NLTL designs, the gate length is scaled down to $0.25 \mu \mathrm{m}$, resulting in a fourfold increase in $f_{c}$, and $\sim 15 \%$ decrease in $C_{\max } / C_{\min }$.

The work on CMOS varactors described up to this point was only the first stage. There was a concern that the varactor models used in the ADS simulation might not extrapolate to high-frequency behaviours. The HP 8722ES vector network analyzer with separated external bias tees was acceptable for this kind of testing but not the best choice, since it makes calibration very difficult. Even through the $26-\mathrm{GHz}$ bias tees were used, the measured data at $20 \mathrm{GHz}$ were found to be noisy. (Only the data below $10 \mathrm{GHz}$ were reasonable - see 'test 2' curve in Figure 4-5).

This part of the work provided guidelines for the subsequent CMOS varactor research, which was done using better simulation strategies and better testing 
equipment. Also the research covered a higher frequency range. The results are presented in the next section.

\subsection{High-Frequency Characteristics of CMOS Varactors}

Fast-developing state-of-the-art CMOS technologies, with cutoff frequencies over $200 \mathrm{GHz}$ [56], have made millimeter-wave silicon RF and MMICs a reality [57], [58]. These devices help fill the demand for low-cost, low-power and compact wireless communication products. CMOS varactors, as key components in many RFICs, have received much attention [55], [56], [57], [58], and [59].

Very few publications have discussed the behavior of CMOS varactors above 50 GHz [57], [58]. Reference [55] gives a very good overview of CMOS varactor structures but covers only up to $5 \mathrm{GHz}$. Reference [59] has a good discussion of CMOS varactors, but the analysis is based on standard foundry-supplied models, which do not normally extend above $20 \mathrm{GHz}$ range. In this work, the CMOS varactor study is extended to $55 \mathrm{GHz}$. The study focuses on four of the six varactor structures in Figure 4-1: They are the AMOS varactor shown in Figure 4-1(1), the IMOS (or SDF) varactor shown in Figure 4-1(3), the NMOS (or SDB) varactor shown in Figure 4-1(4), and the SnDp varactor ( $N$-type source, $P$-type drain) shown in Figure 4-1(6). As pointed before, the first two structures have monotonic $C(V)$ curves, and the last 
two have non-monotonic $C(V)$ curves. All these four structures were fabricated on a TSMC CMOS 0.18-mm chip.

The fabricated structures were tested using an $\mathrm{HP}$ 4280A 1-MHz capacitance meter for the low-frequency parameters. For GHz-range measurements, an Agilent N5250A Performance Network Analyzer (PNA) with built-in bias tees, a Karl Suss PA 200 probe station, and programmable heads with Picoprobe GSG-67A-100 probes were used. The measurement setup is shown in Figure 4-6. On-wafer measurements were extracted up to $67 \mathrm{GHz}$ but for accuracy, post-measurement calculations were carried only to $55 \mathrm{GHz}$. A CS-5 ceramic-substrate kit was used to set the measurement reference plane to the tip of the probes. Koolen's technique [68] was used to de-embed the extrinsic parameters due to use of pads and interconnects. The 2-port admittancematrix parameters of the varactor (DUT in Figure 4-6) were extracted by using "open" and "short" correction structures. More calibration and de-embedding details for highfrequency measurements are given in Appendix B. 


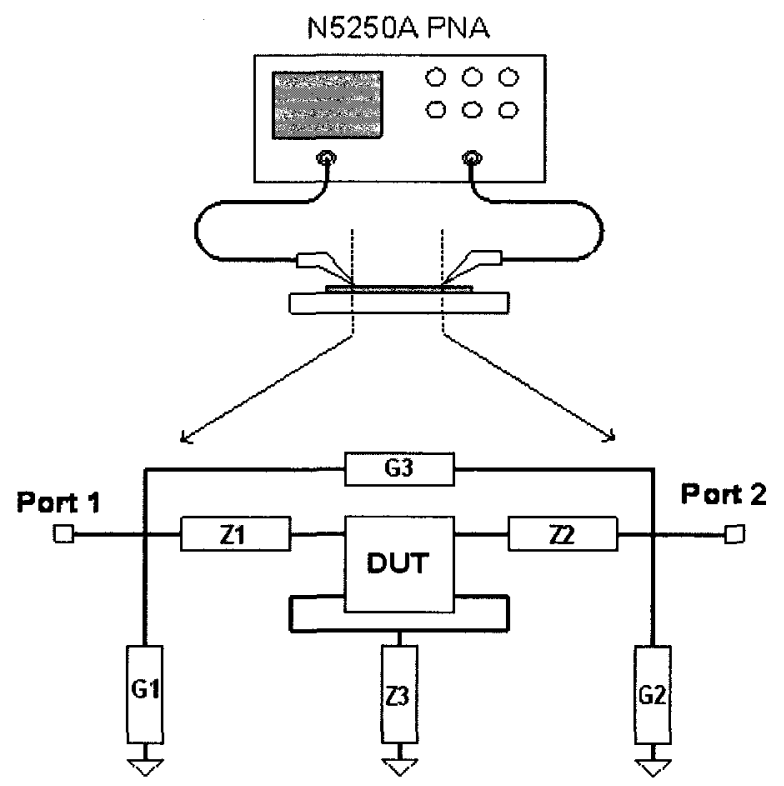

Figure 4-6 The CMOS varactor measurement and de-embedding setup.

The four CMOS varactor types are also simulated up to $55 \mathrm{GHz}$ by using the process-oriented device simulator Medici [52]. This simulator is able to predict the electrical characteristics of devices from user-specified structures, including material properties and doping profiles. To examine the high-frequency behaviour of the CMOS varactors, the tested and simulated $C(V)$ and cut-off frequency curves of the four kinds of fabricated CMOS varactors were obtained at $1 \mathrm{MHz}, 5 \mathrm{GHz}, 20 \mathrm{GHz}$, and $55 \mathrm{GHz}$. Figure 4-7 shows the results for the AMOS varactor. The left column contains the simulated data, the right column the measured data. The top row shows the $C(V)$ curves, the bottom row the cutoff frequencies. Similarly, Figure 4-8 shows the results for the IMOS varactor, Figure 4-9 for the NMOS varactor, and Figure 4-10 for the $\mathrm{SnDp}$ varactor. 

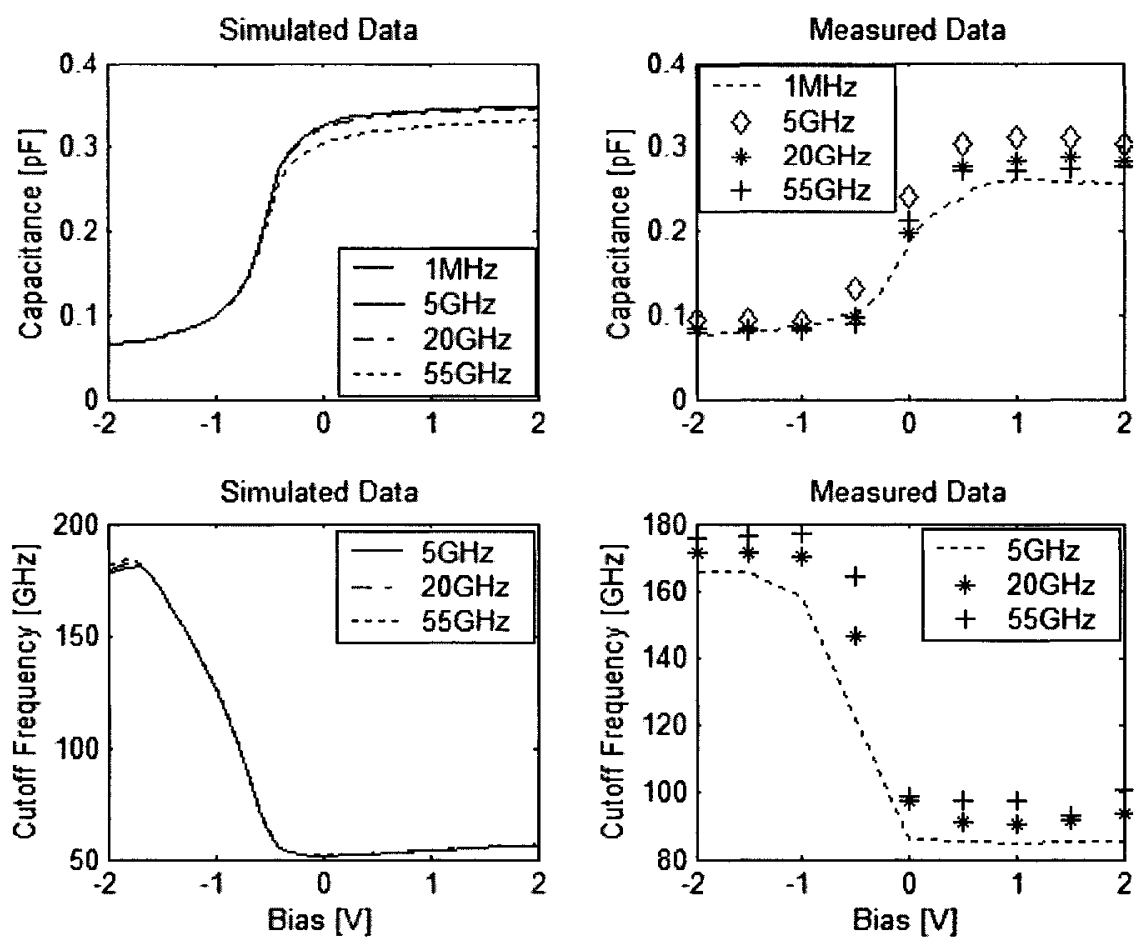

Figure 4-7 Simulated and measured data for the AMOS varactor.
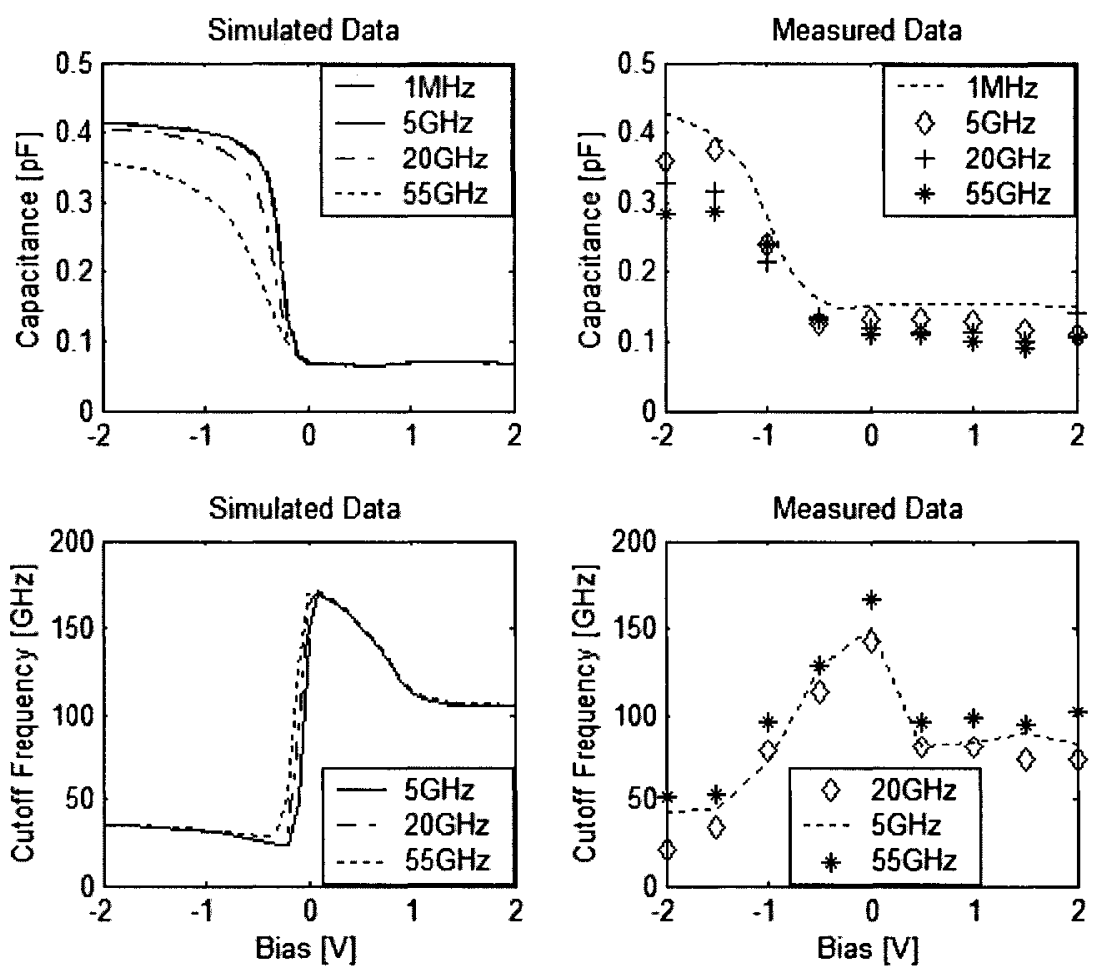

Figure 4-8 Simulated and measured data for the IMOS varactor. 

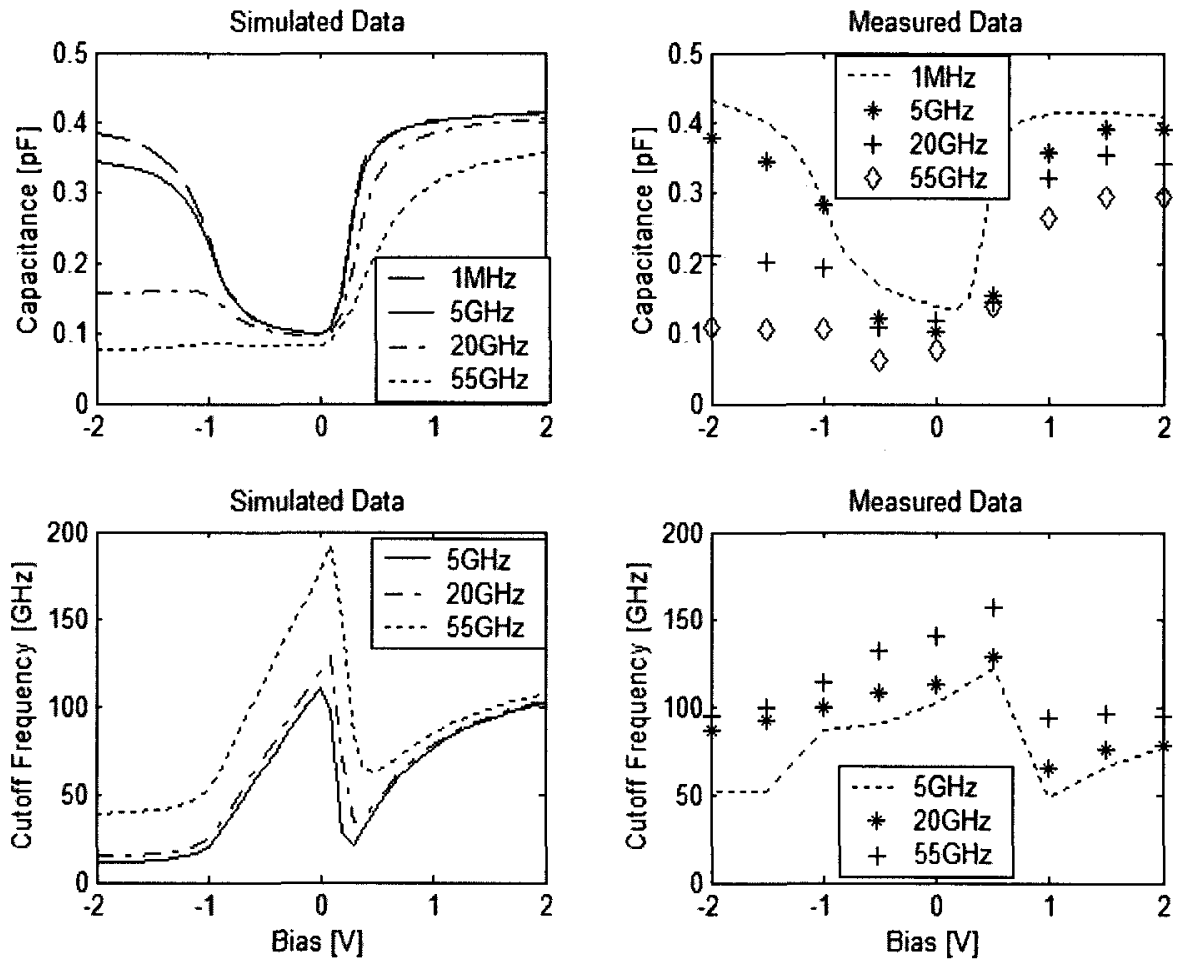

Figure 4-9 Simulated and measured data for the NMOS varactor.
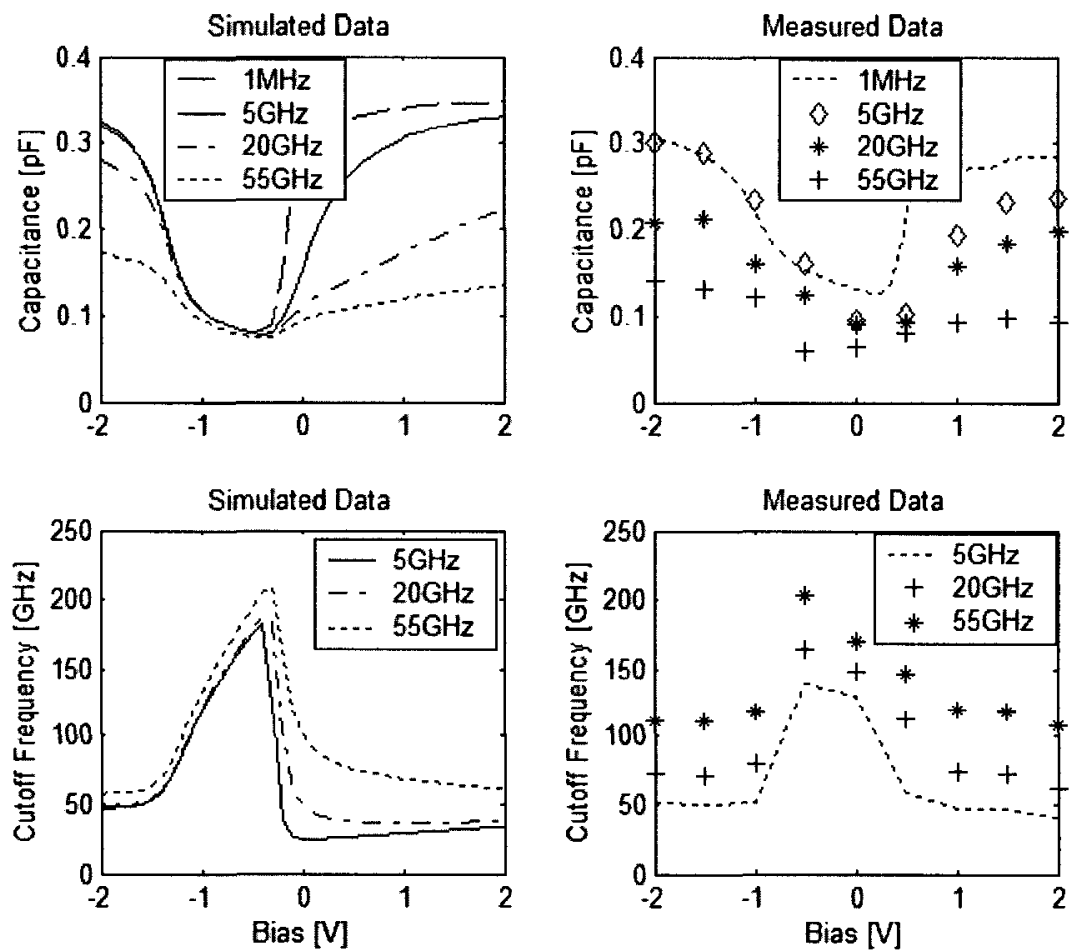

Figure 4-10 Simulated and measured data for the $\mathrm{SnDp}$ varactor. 
The AMOS varactor data of Figure 4-7 show that on the average, the simulated $C_{\max } / C_{\min }$ ratios are $\sim 3.8$ while measured values are $\sim 3.4$. The simulated cutoff frequencies are $\sim 135 \mathrm{GHz}$ while measured values are $\sim 130 \mathrm{GHz}$. The IMOS varactor data of Figure 4-8 show that on the average both the simulated and measured $C_{\max } / C_{\min }$ ratios are $\sim 3$. Also, the cutoff frequencies are both $\sim 100 \mathrm{GHz}$. The small differences between simulation and measurement are attributed to discrepancies between simulation settings and real process values. Of particular interest is the frequency-dependence of the $C(V)$ curves. For the AMOS varactor, see Figure 4-7, the simulated $C(V)$ s vary little from $1 \mathrm{MHz}$ to $55 \mathrm{GHz}$, whereas the measured highfrequency values are slightly higher than those at $1 \mathrm{MHz}$. This may be due to the 1$\mathrm{MHz}$ measurements being taken using a capacitance meter, whereas the PNA was used for the $5-55 \mathrm{GHz}$ measurements. For the IMOS data of Figure 4-8, the simulated and measured $C(V)$ curves both show decreased nonlinearity as the frequency increases. The reason for this degeneration is that the IMOS varactor has a larger channel resistance than the AMOS varctor, since in the IMOS structure the well makes no contribution to the channel conductivity.

The NMOS and SnDp varactors have non-monotonic $C(V)$ characteristics. Figure 4-9 shows that on the average, the NMOS varactor simulations predict $C_{\max } / C_{\min }$ ratios of $\sim 3.6$ compared with measured ratios $\sim 3.4$. However both simulated and measured 
$C(b)$ curves degenerate towards monotonicity as the frequency increases. The simulated average cutoff frequencies are $\sim 100 \mathrm{GHz}$ while the measured values are $\sim 95$ GHz. Figure 4-10, for the $\mathrm{SnDp}$ varactor, shows that at lower frequencies, the average simulated $C_{\max } / C_{\min }$ ratios are $\sim 3.0$ and the measured ratios $\sim 2.7$. Both simulated and measured $\mathrm{SnDp} C(V)$ curves are flattened in the 20 -to-55 $\mathrm{GHz}$ range, with $C_{\max } / C_{\min }$ ratios dropping dramatically. In Figure 4-10, the average cutoff frequencies are all $\sim 100 \mathrm{GHz}$.

Comparing Figure 4-5 to Figure 4-7, 4-8, 4-9 and 4-10 it can be seen that using Medici to simulate CMOS varactor is better than using varactor model through ADS. The results from Medici have better agreement with measured data. Also, the VAN with built-in bias tee gives more reliable testing results.

On the chip, the gate of each varactor has a length of $0.5 \mu \mathrm{m}$, a width of $5.0 \mu \mathrm{m}$, and a total of 12 fingers. In the simulation setup, the Medici software's default and normalized width is $1.0 \mu \mathrm{m}$, and we set the length to $0.5 \mu \mathrm{m}$. Therefore, the simulated capacitance was multiplied by 60 in order to compare it with the measured data. The cutoff frequencies should not be affected by the number of fingers. 
The relatively poor high-frequency response of the SnDp structure is probably due to the need for carriers to travel the full length of the channel from the $\mathrm{S}$ or $\mathrm{D}$ region. In the other structures, carriers need only travel half the length of the channel. To test this contention, we also simulated NMOS and SnDp varactor structures at $55 \mathrm{GHz}$ with the gate length shortened to $0.2 \mu \mathrm{m}$. Figure $4-11$ compares the $0.2-\mu \mathrm{m}$-gate results with those obtained for the same devices with $0.5-\mu \mathrm{m}$ gates. For this comparison, all capacitances are normalized to the same gate area. Figure 4-11 shows that with short gate length, the $\mathrm{SnDp}$ varactor has reduced $C(V)$ degeneration and a much higher cutoff frequency than the NMOS varactor.
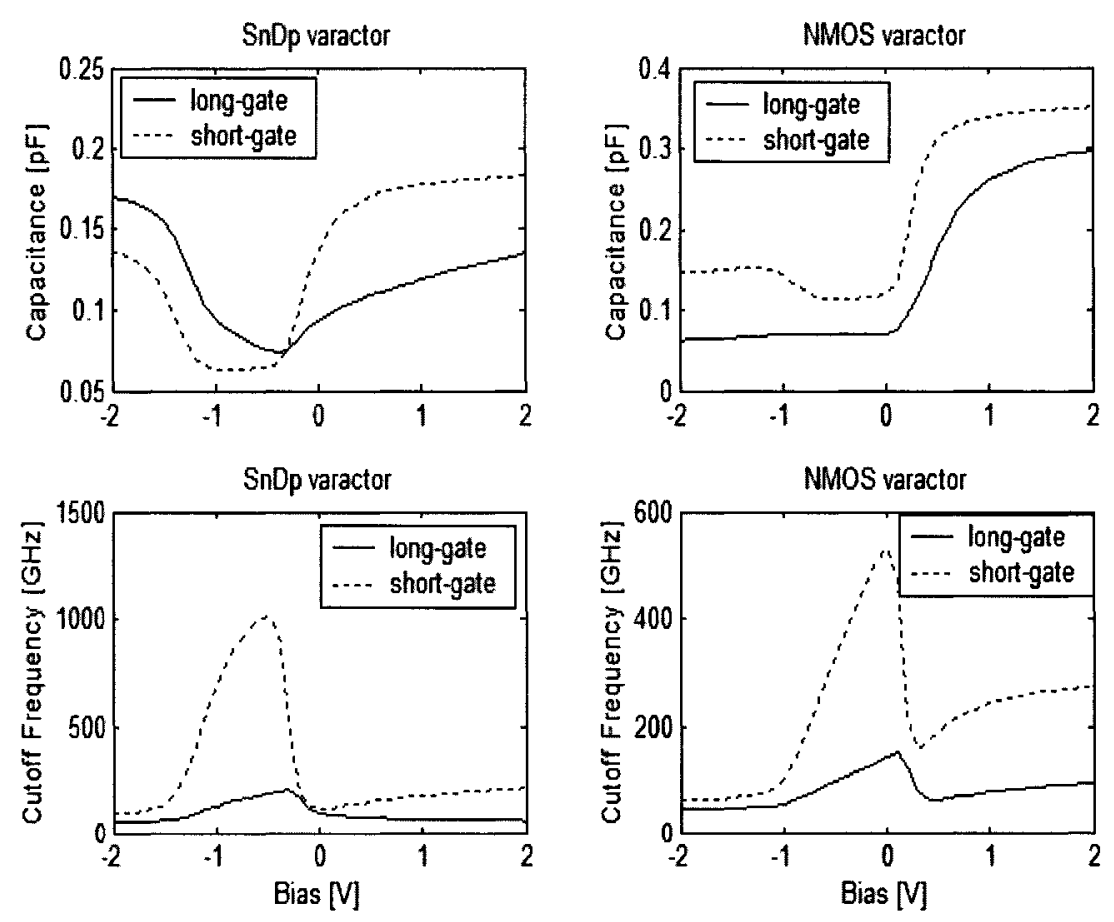

Figure 4-11 SnDp varactor versus NMOS varactor at $55 \mathrm{GHz}$ (short-gate $=0.2 \mu \mathrm{m}$, long-gate $=0.5 \mu \mathrm{m})$. 
For a further comparison, Figure 4-12 shows the series resistances for AMOS, SDF and SDB varactors at $5 \mathrm{GHz}$. A gate area of $0.5 \times 12 \mu \mathrm{m}^{2}$ was used for all the three varactors. A unit "Ohms" was used to measure the resistances for the entire device because it is improper to normalize the varactor series resistance to the gate area with the complicated MOS varactor models [60], Figure 4-12 indicates that among these three kinds of varactors, the AMOS type has the lowest series resistance, and the SDF type has the highest series resistance. The low resistance value of the AMOS varactor is reasonable when compared to that in [60]. The explanation is that in the AMOS varactor structure, since $\mathrm{n}+$ diffusions are in an $n$-well, there are fewer parasitic junctions and paths to ground, resulting in higher $Q$ and lower resistance. In the case of the SDF (or IMOS) structure, the source and drain, although connected together, are not connected to the well. Because of this, the well makes no contribution to the channel conductivity (as pointed out before), which makes the resistance higher. It is seen in Figure 4-12 that both the SDF and SDB have their resistance peak value around the depletion region. This is because, in the depletion region, the hole-electron combination rate reaches its maximum value, and leaves no free charge carriers in the channel. But with the AMOS varactor, since there are no minority carriers in the channel, the device never reaches a deep depletion mode. Therefore, for AMOS varactors, there is no resistance peak near the depletion region. This is a big advantage for AMOS varactors. 


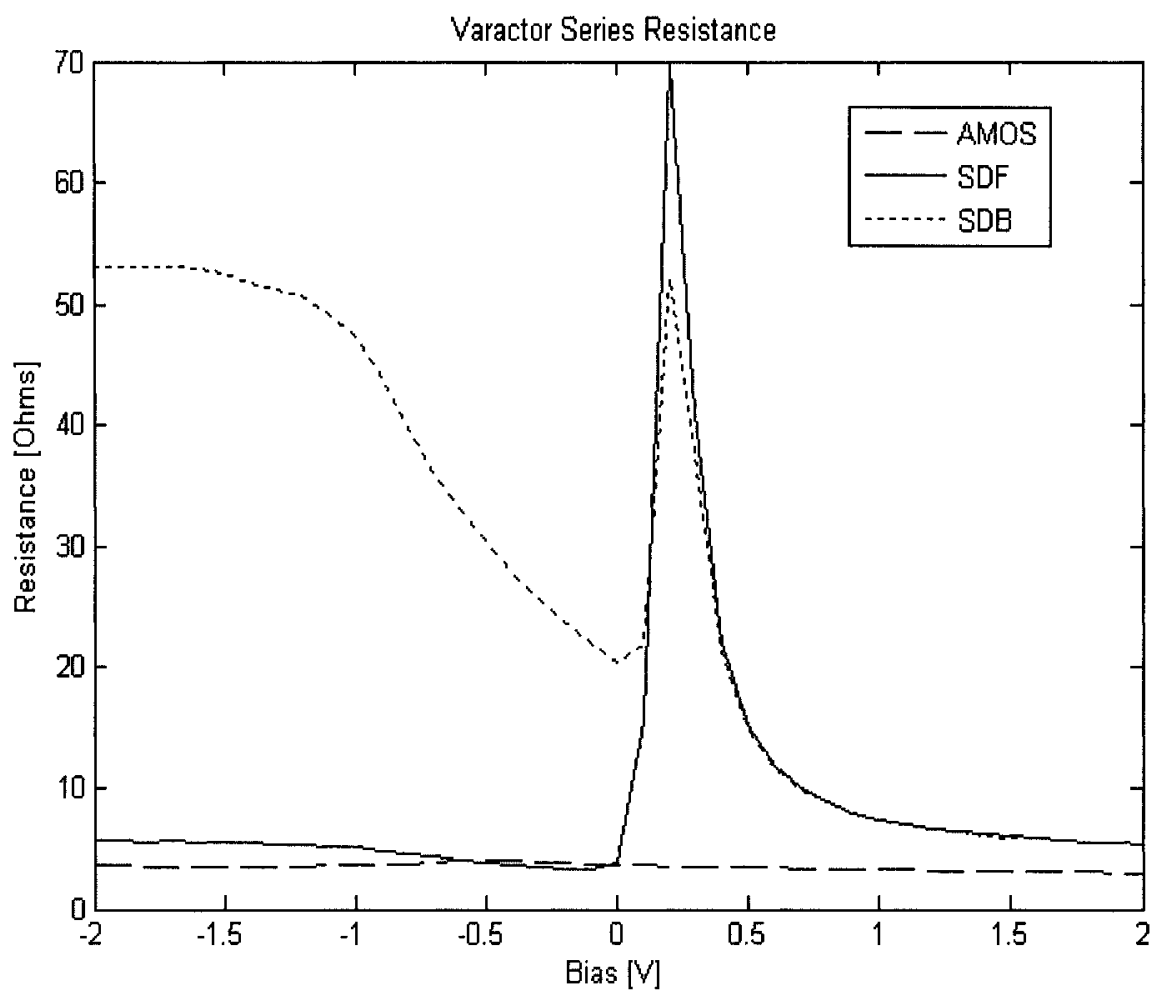

Figure 4-12 Series resistances at $5 \mathrm{GHz}$ for AMOS, SDF and SDB varactors with gate area $=0.5 \times 12 \mu \mathrm{m}^{2}$.

\subsection{Conclusion}

In this chapter, six CMOS varactor structures are investigated and discussed. Both measured and simulated data for the six structures are presented and compared. These research results show that both AMOS and IMOS varactors are good for single-edge pulse compression due to their higher $C_{\max } / C_{\min }$ ratios and cutoff frequencies. However, the AMOS varactor has advantages over the IMOS (or SDF) varactor because its $C(V)$ curve does not degenerate at higher $\mathrm{GHz}$ frequencies, and its series 
resistance is smaller than that of the IMOS varactor. Both NMOS (or SDB) and SnDp varactors are good for double-edge pulse compression due to their non-monotonic $C(V)$ curves. However, the high-frequency degeneration restricts their use to a much lower $\mathrm{GHz}$ range. Using a short-gate $\mathrm{SnDp}$ varactor can reduce its high-frequency degeneration and improve its performance. 


\section{Chapter 5 All-Silicon Pulse-Compression NLTL}

\section{Design}

\subsection{Design Strategies}

A critical condition for a correctly-functioning pulse-compression NLTL is that the ratio of the varactor cutoff frequency $f_{c}$ to the Bragg cutoff frequency $f_{B}$ should be $\sim 5$ [20]. For a 50- $\Omega$ NLTL, a CPW line with $Z_{0}>50 \Omega$ is needed. This is because the varactors capacitively load the CPW line, thereby reducing the $Z_{0}$. To get sufficient NLTL nonlinearity, the varactors need a large $C_{\max } / C_{\min }$ ratio. This ratio depends on the CMOS varactor structure. Also, $C_{\min }$ must be large enough to overcome the linear capacitance-per-section of the NLTL. Tapered NLTLs are used for loss minimization [61]. When a pulse propagates along an NLTL, its rising and/or falling edges become sharper, so that the signal bandwidth progressively increases. In the so-called tapering technique, the NLTL section length and varactor gate width are both continuously decreased along the transmission line, thereby correspondingly increasing the Bragg cutoff frequency.

The tapering structures can be linear or non-linear. The nonlinear example is an exponential tapering structure [62], in which the diode capacitances $C_{\mathrm{n}}$ and the diode spacings $L_{\mathrm{n}}$ were tapered following an exponential trend as 


$$
C_{n}=C_{1} \cdot a^{n-1} \quad \text { and } \quad L_{n}=L_{1} \cdot a^{n-1},
$$

where $C_{1}$ and $L_{1}$ are the first capacitor and section length of the NLTL, $a$ is the taper factor, and $n$ is the index. In this work, the tapering function is simplified to a linear type. The tapering equations are presented as

$$
L_{n+1}=\left(1-n \cdot K_{1}\right) \cdot L_{n} \quad \text { and } \quad W_{n+1}=\left(1-n \cdot K_{2}\right) \cdot W_{n}
$$

where $L$ is the NLTL section length, $W$ is the varactor gate width, $n$ is the section index, and $K_{1}, K_{2}$ are taper factors.

Another way to explain this is to say that the tapered NLTLs are designed such that the Bragg cutoff frequency $f_{B}$ increases gradually along the line, thereby coping with the increasing bandwidth of the propagating signal. Also, the NLTL losses are reduced by using progressively shorter CPW section lengths and smaller-size varactors.

In all-silicon NLTL designs, the greatest challenge is the loss in the silicon substrate. Here slow-wave CPWs are used as NLTL linear elements to overcome the substrate loss. The NMOS SDF and SDB varactors are used as NLTL nonlinear 
elements to do single-edge or double-edge pulse sharpening, since they are available components in the standard CMOS process.

Figure 5-1 gives a flow chart of the all-silicon NLTL design process. The Synopsys Medici [52], Ansoft HFSS [50], Sonnet [43], Agilent ADS [44] and Cadence software packages were used for the pulse-compression NLTL simulations and designs. These packages are briefly described as follows:

- Medici is a process-oriented device simulator. The simulator is able to predict the electrical characteristics of devices from user-specified structures, including material properties and doping profiles. It is suitable for evaluation and characterization of CMOS varactor structures.

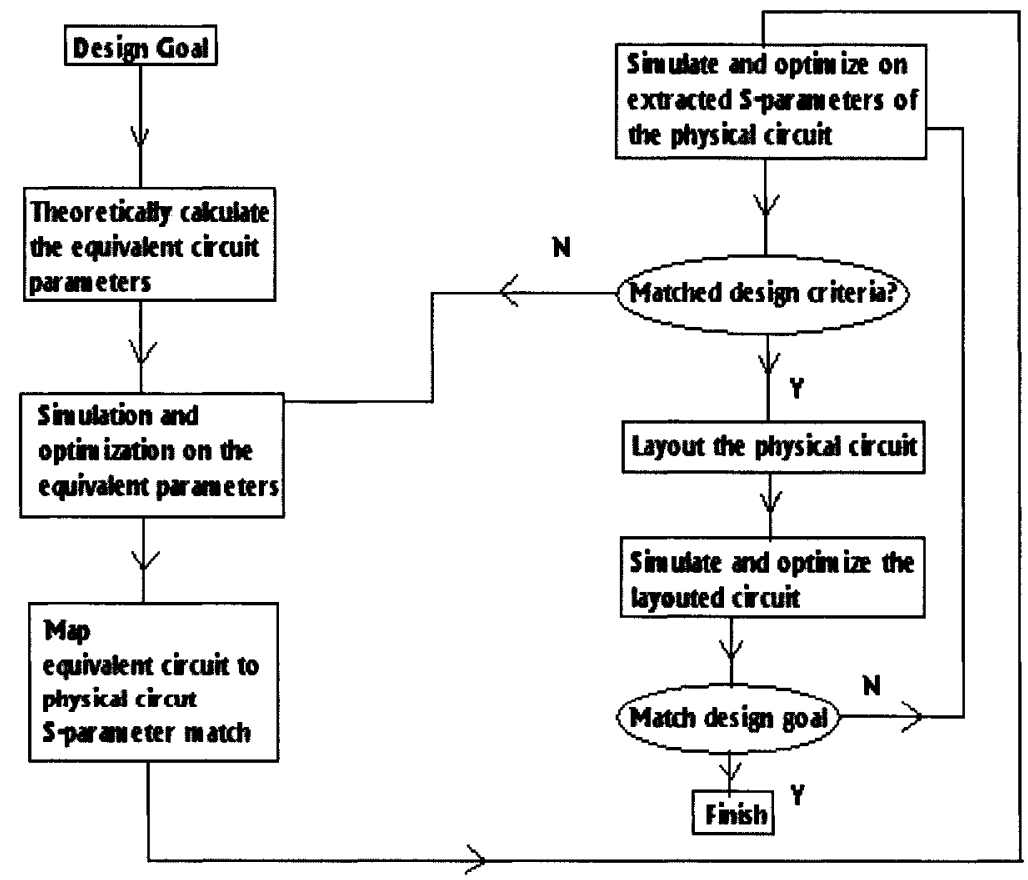

Figure 5-1 Flowchart of the silicon NLTL design process. 
- HFSS is an industry-standard high-frequency simulation program. It can be used for $S$-parameter extraction and 3-D full-wave $E M$ (electromagnetic) field simulation. Also, it can provide high accuracy, large capacity, and good performance when designing high-frequency and high-speed components. In this study, HFSS was used for characterizing and optimizing the silicon CPW and silicon slow-wave CPW lines.

- Sonnet is also a high-frequency electromagnetic program. Similar to HFSS, Sonnet can analyze 3-D EM field configurations and develop RF models with $S$-, $Y$-, Z- parameters. However, Sonnet is sometimes called "2.5-D" software because it can only deal with planar circuits. The advantages of this software are that it requires much shorter simulation times than HFSS, and the setups are simpler and more straightforward. In this study, Sonnet was used for the first-stage evaluation and in producing the initial simulation values for HFSS.

- ADS is a powerful electronic design-automation software system. It offers complete design integration for RF and wireless communication networks. ADS can import component $S$-parameters from Medici, HFSS, and Sonnet. Here, ADS was used to integrate the CPW and varactor components, and to design the NLTL circuits. 
- Cadence is a semiconductor design system. It provides front-to-back design tools and a standard interface to the chip manufacturers. The Cadence design tool was used for the NLTL chip layout. The NLTL lines were built on a standard CMOS $0.18-\mu \mathrm{m}$ microchip through the facilities of CMC Microsystems Inc.

\subsection{NLTL Chip Layout}

The NLTLs were fabricated on standard $0.18-\mu \mathrm{m}$ CMOS chips. There are two kinds of components included in the circuits, CMOS varactors and silicon CPWs. The vias on the chips were used to make the connections between the varactors and the CPW lines. Figures 5-2 and 5-3 give simplified top and cross-sectional views of a NLTL layout. Note that the figures are not drawn to scale. 


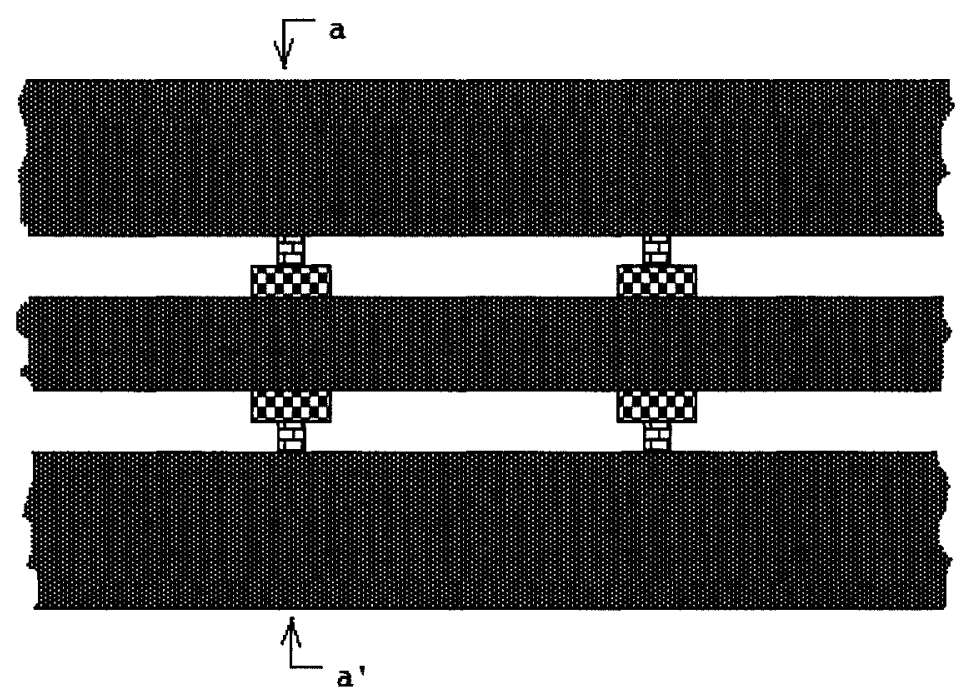

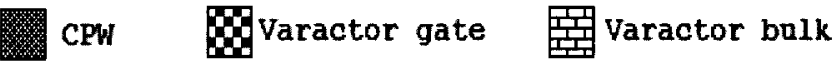

Figure 5-2 Top view of a CMOS NLTL layout (not to scale).

Figure 5-2 is top view of a CMOS NLTL layout. CMOS varactors were connected to a CPW line symmetrically on the both sides of the CPW signal line. This will keep the NLTL from working in coplanar-strip-mode (the two CPW ground planes at different potentials), as pointed out in Chapter 2. 


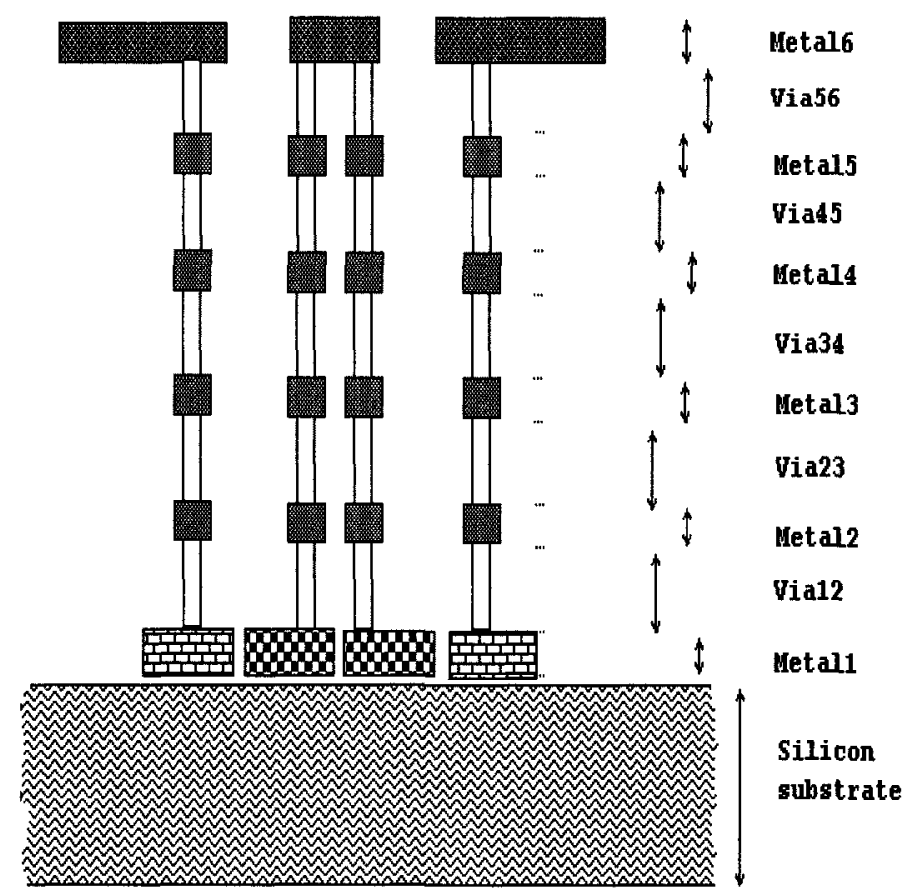

CPH Maractor gate 置 varactor bulk

Figure 5-3 Cross-sectional view of the CMOS NLTL shown in Figure 5-2 at plane a-a' (not to scale).

Figure 5-3 is cross-section view of the CMOS NLTL. It was mentioned in Chapter 2 that certain layout strategies can be used to reduce layout-produced parasitics. Increasing the density of the connecting vias can reduce inductive parasitics.

\subsection{Simulated NLTL Waveforms}

Simulated waveforms for the all-silicon slow-wave pulse-compression NLTL design are shown in Figure 5-4. The simulations were done by using the ADS 
simulator. Measured data for the fabricated CMOS varactors and silicon slow-wave CPW lines were used in the simulation. In other words, the simulated results were based on the measured component data.

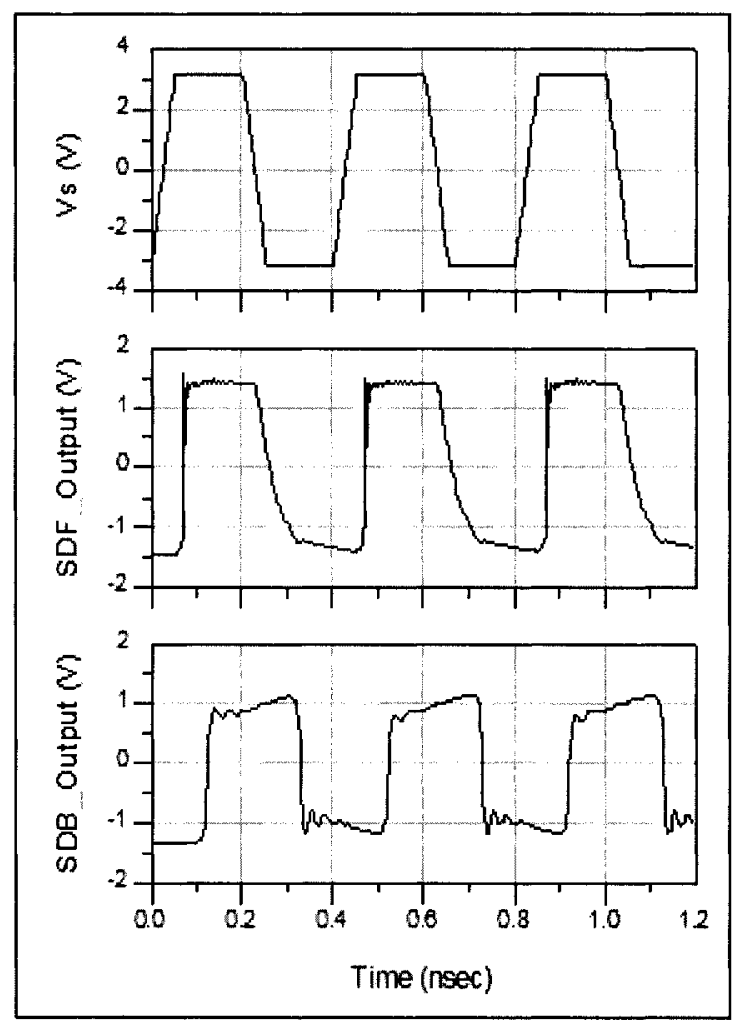

Figure 5-4 Simulated slow-wave-NLTL waveforms using measured S-CPW and varactor data.

For the first section of the NLTLs, the CPW signal-line width is $10 \mu \mathrm{m}$ and the gap between the signal and the ground line is $45 \mu \mathrm{m}$. Each varactor gate finger is 0.25 $\mu \mathrm{m}$ long by $2.5 \mu \mathrm{m}$ wide, and there are 30 fingers. Since the tapered technique was used, taper factors are selected to get maximum NLTL efficiency. Here, for the SDFNLTL, the section-length taper factor is 0.06 . The same number is used for the 
varactor gate width. For the SDB-NLTL, the section-length taper factor is 0.06 , and 0.05 for the varactor gate width. The entire lengths of the SDF and SDB NLTLs are $2.7 \mathrm{~mm}$ and $3.4 \mathrm{~mm}$ respectively. Both NLTLs have 16 sections. The $10-90 \%$ rise and fall times of the input were set at 40 ps. Figure 5-4 shows that the output rise time of the SDF NLTL is about 10 ps, which represents $75 \%$ compression. While the output

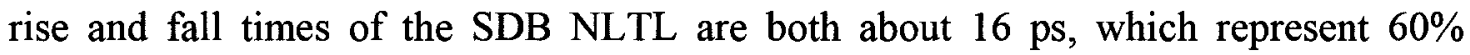
compression.

\subsection{Summary}

This chapter discusses strategies for designing pulse-compression NLTLs, which include using the tapered technique, simulating with various commercial software packages, and experimenting with different layout designs. The simulated results based on the measured CPW and varactor data are also presented in this chapter. These simulated results demonstrate that SDF varactors are especially good for singleedge compression and that SDB varactors are especially good for double-edge compression. The $75 \%$ reduction in the rise time for single-edge compression and $60 \%$ reduction on both rise and fall times for double-edge compression indicate it should be very possible to build effective pulse-compression NLTLs on standard silicon chips. 


\section{Chapter 6 Device Measurements}

Based on the analysis, modeling, and simulation, the NLTL circuits, including their linear CPW and nonlinear varactor components, were realized on CMOS ICs. A CMOS $0.18-\mu \mathrm{m}$ process was used as the study platform. In this study, frequencies of the input signals are in the range of 5 to $10 \mathrm{GHz}$. The output signals can reach to 50 $\mathrm{GHz}$ due to enriched harmonics, which means that the output waveform can have transient times in the picosecond range. This makes the measurements very difficult. The measurements can be divided into two parts: small-signal frequency-domain testing and large-signal time-domain testing.

\subsection{Small-signal Frequency-domain Testing}

Small-signal frequency-domain testing includes $S$-parameter measurement. $S$ parameters are very useful for small-signal modeling in situations where the values of equivalent elements can be verified. They are also very useful for determining NLTL insertion loss, characteristic impedance and cutoff frequency. Vector network analyzers (VNA) are able to measure the amplitude as well as the phase of the $S$ parameters of the device under test (DUT). A schematic representation of a VNA is shown in Figure 6-1. The VNA includes an RF generator. The ports are measured in turn, when the source is switched from one port to another. When one port of a 2-port 
is connected to the generator, the other is switched to a matched load. For example, when switch is in the "I" state, the port on the left is connected to the generator, the other to the matched load. The delivered signal is then split into two parts. The first part, denoted RA or RB, is an image of the incident wave, while the second part propagates toward the DUT. Directional couplers are used to recover the reflected part of the signal (TA) and a part of the signal that came out of the DUT (TB). However, the measured $S$-parameters are not the real $S$-parameters of the DUT, since the reference plane of the VNA can be far from the edge of the DUT. The DUT is usually connected to the VNA through long coaxial cables, represented in Figure 6-1 as boxes $\mathrm{A}$ and B. As a consequence, the measured $S$-parameters must be corrected. This operation can be done by using some specific techniques to calibrate the VNA and then de-embed undesired passive data.

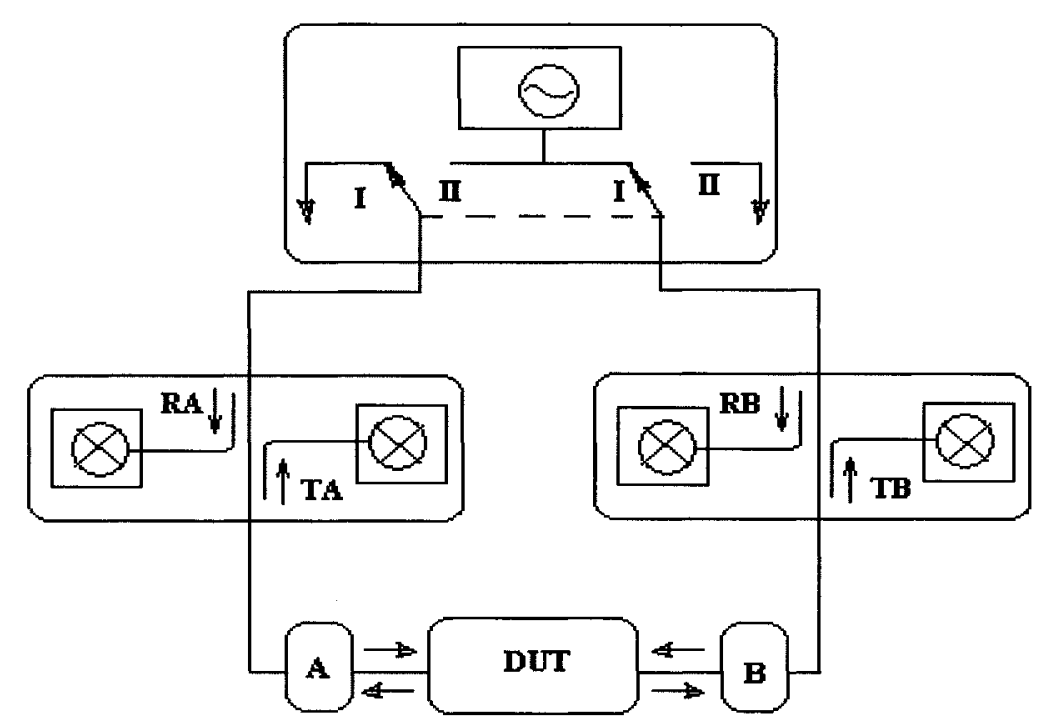

Figure 6-1 Principle of operation of a VNA. 
The term "on-wafer measurement" refers to the measurement of integrated devices directly on the wafer, immediately after fabrication, before slicing and encapsulation. It is the most reliable method to characterize integrated devices, since a minimum of parasitics in involved. To carry out on-wafer measurements, the wafer is placed on a specific probe station, where mechanical arms allow tips to be connected to the device. The probes are connected to the VNA through coaxial cables, as shown in Figure 6-2. The probes are essentially transitions from a (large) coaxial cable structure to a (small) CPW structure. Figure 6-3 shows an on-wafer ground-signal-ground (GSG) probe and the probe connecting to a CPW line.

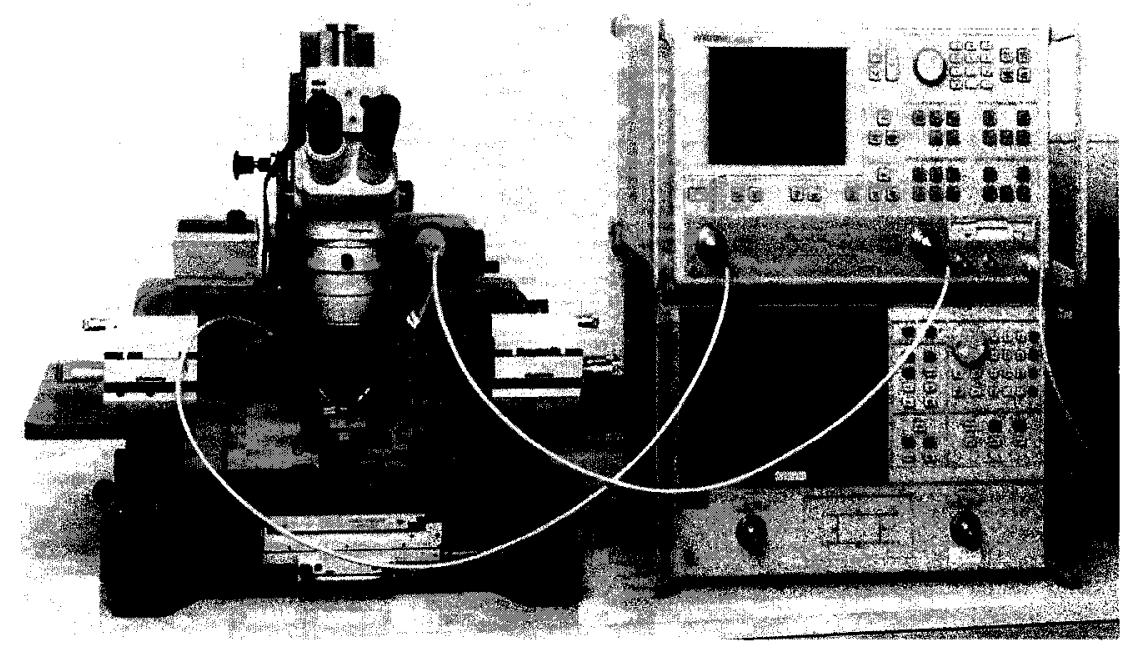

Figure 6-2 On-wafer probing station connected to a VNA. 


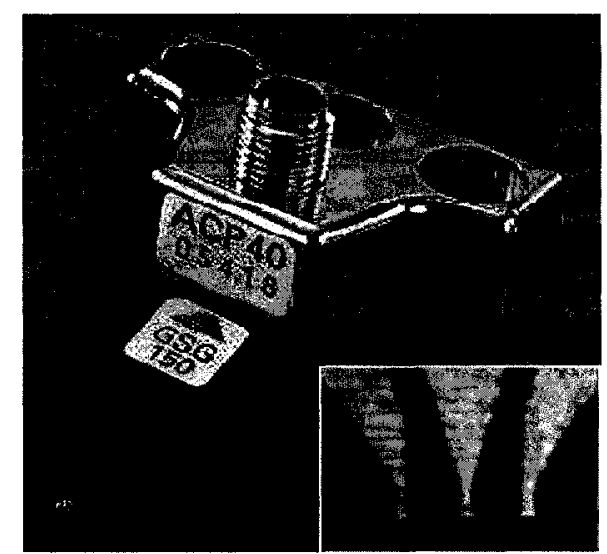

(a)

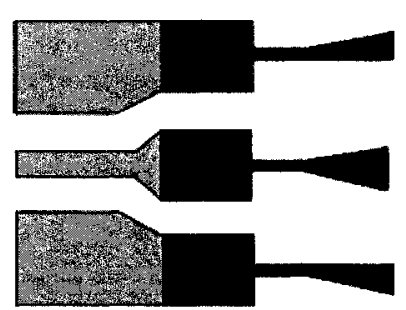

(b)

Figure 6-3 (a) An on-wafer GSG probe. (The inset shows a microscopic view of the probe tips) (b) An artist's view of a probe (black) contacting a CPW structure (grey).

In order to perform accurate on-wafer measurements, an on-wafer calibration and de-embedding scheme is required. The technique called "two-stage calibration" [63] can be used for CMOS on-wafer measurement. It is described below.

(1) On-wafer calibration

On-wafer calibration is the first stage of the CMOS measurement process. It is used to determine the number of error coefficients caused by connecting cables that must be extracted by calibration. The on-wafer calibration must be done by using standards fabricated on the wafer [63]. There are three commonly used selfcalibration methods: Short-Open-Load-Through (SOTL), Through-Reflect-Line (TRL), and Through-Reflect-Match (TRM). Appendix B gives more detail on these three calibration methods. The TRM method is not frequency-limited but this algorithm requires the measurement of two perfectly matched loads (one for 
each port). In CMOS technologies, it is not possible to achieve the required match between loads. Indeed, variations close to $10 \%$ of the DC values of resistances made on a CMOS process have been observed. Therefore, TRM cannot be implemented on a silicon wafer. Instead, a commercial calibration substrate, made on an alumina substrate, having a load which could tolerate variations less than $0.1 \%$, can be used for an accurate calibration up to $40 \mathrm{GHz}$ [63]. For an alternative, the TRL method can be used. However, as the method is frequency-dependent, we have to know the reference impedance. In practice, to minimize the frequency limitation of the TRL, more TRL lines have to be built on a substrate calibration chip, which takes extra space. In this work, based on cascade configurations, a planar standard, known as CS-5, and the SOTL technique are used to estimate the effects of surrounding parasitic networks on a CMOS device, which can be either a CPW line or a CMOS transistor. After this calibration, the reference planes move to the end of the probe tips, and the reference impedance is equal to $50 \Omega$.

\section{(2) Passive de-embedding}

When measuring a passive device, the pads, feeding lines and other components used to facilitate on-wafer probing can corrupt the measurements with unexpected errors. A passive de-embedding process determines and removes the probe-pad parasitics, which are introduced by the pads fabricated on the silicon wafer. There are a few techniques that can be used to de-embed the effects. In many cases, the TRL technique is used. The standards are built on-chip together with the DUT. There are two CPW lines, and a reflective device. The latter can be an open or a 
short circuit. The CPW is designed to have a characteristic impedance of nearly 50 $\Omega$. Then the line length is chosen in order to have the most accurate measurement in the frequency band of interest. If the validity of the calibration must be extended to nearly the whole frequency band, multiple lines have to be used. The TRL structure is shown in Appendix B, Figure B-1. After this calibration, the reference planes are shifted to the middle of the through-line, or to the ports of the DUT under measurement.

When the measured devices have small dimensions, as do integrated submicron transistors and CPWs, it is possible to use methods other than the on-wafer TRL approach. One method, called admittance corrections, was first proposed by van Wijnen for bipolar devices [64] and then enhanced by Fraser [65]. Its validity has been recently demonstrated by Vandamme [66] using three-step de-embedding for deep submicron MOSFETs up to $40 \mathrm{GHz}$. This method is based on an admittance equivalent circuit of the DUT and the probing structure. In order to extract the different parasitic elements, specific de-embedding structures are measured through open and short circuits. All these structures are designed in order to mimic the same parasitics. The different open and short circuits utilized for the calibration are assumed ideal. Therefore, this algorithm cannot be applied to large devices at high operation frequencies. In addition, there is a three-step open-short method introduced by Cho [67], a two-step technique presented by Koolen [68], and a one-step $A B C D$ cascaded matrix method created by Heymann [69]. 
In theory, the three-step de-embedding can provide more accurate results since more parasitic components are considered. However, this method needs more chip space since four de-embedding structures need to be put on the chip together with the DUT. Also, it needs more testing steps and each step can introduce additional errors. On the other hand, Koolen's method may be not very precise but is simpler and more straightforward, and needs less chip space since only two de-embedding structures have to be put on the chip. More details about these de-embedding methods are given in Appendix B.

In this work, the CMOS varactors, CPWs, NLTLs, and their corresponding deembedding standards were fabricated in a standard CMOS process and characterized up to $40 \mathrm{GHz}$. The $\mathrm{CPW}$ and varactor $S$-parameters were measured up to $40 \mathrm{GHz}$ using an HP 8722ES vector network analyzer (VNA) and Picoprobe ground-signalground (GSG) probes. After investigating and comparing different kinds of calibration and de-embedding techniques, Koolen's technique was used to de-embed the extrinsic parameters caused by pads and interconnects.

\subsection{Large-signal Time-domain Testing}

Large-signal testing is critical for capturing the nonlinear behavior of NLTLs. Until now, measuring the nonlinear behavior of RF components was not easy. $S$ parameters were developed to analyze and model the linear behavior of RF devices in 
the frequency domain. They have limitations and are defined only for small-signal linear systems. Large-signal time-domain measurement is more complicated than small-signal testing. A high-speed oscilloscope is required to capture fast-transition waveforms. Figure 6-2 shows the measurement setup used for conventional NLTL time-domain testing, where both input and output signals are sent to a high-speed oscilloscope in order to compare their rise and fall times. A 3-dB power splitter was used to equally divide the input power into two paths. The two dc bias-tees were used to set different bias voltages at the NLTL device. The amplitude of the input at the tested device has to be less than 3 volts since the maximum voltage for a CMOS 0.18$\mu \mathrm{m}$ chip is 3.3 volts. The challenging aspects are producing high-speed pulse inputs and calibrating the signal propagation delays of the test instrument and connecting wires. Even more difficult is to find an oscilloscope for frequencies $>50 \mathrm{GHz}$, which can capture the rise and fall times, of the order of 10 to 20 picoseconds, of the testing pulse signals. 


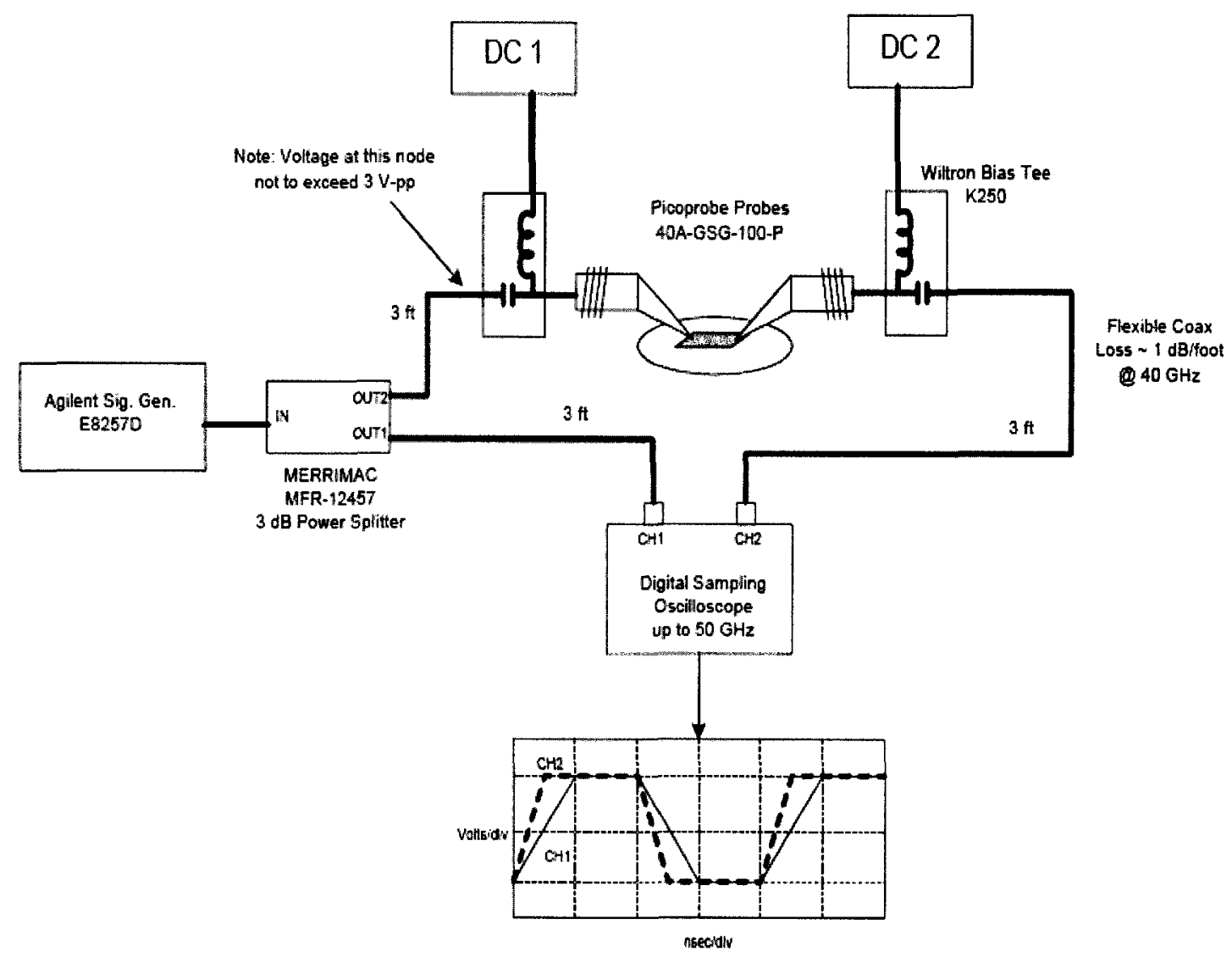

Figure 6-4 NLTL time-domain measurement setup using an oscilloscope.

How is one to produce a scattering or $S$-parameter equivalent that can handle largesignal nonlinear circuits? A few years ago, Root and his associates at Agilent Technologies Inc. started work on this problem. They have now developed the $X$ parameters concept [70]. Mathematically, $X$-parameters are rigorous supersets of $S$ parameters. But unlike $S$-parameters, $X$-parameters can be used to represent and analyze the nonlinear behavior of RF components in a much more robust and complete manner. $X$-parameters include the magnitude and phase characteristics of the device- 
generated spectral components corresponding to distortion, which can include harmonics and inter-modulation products. Phase-normalized quantities and the harmonic superposition principle are used in $X$-parameter theory. More details are given in Appendix B.

$X$-parameters of a nonlinear device now can be measured by using the new Agilent Nonlinear Vector Network Analyzer (NVNA), which is capable of nonlinear calibration and measurements. The measured $X$-parameters can be characterized and extracted by the Agilent design software ADS2008 [71]. This new version of the ADS simulator includes an auto-configurable nonlinear frequency-domain simulation 'PHD block' component, which converts the measured datafile into a device-specific PHD block instance. Hence, it is possible to deduce NLTL large-signal outputs from frequency-domain measurements. The measured results are then converted into timedomain waveforms. The frequency-domain and time-domain responses of the measured nonlinear device can be obtained for further simulation and design. A simplified representation of the NVNA and its interoperability with ADS is shown in Figure 6-3. The related work has been published in $[72,73,74]$.

Since nonlinear VNAs such as the Agilent N5242A are now available, large-signal nonlinearity testing has become more feasible and convenient. The nonlinear VNA can not only measure harmonic amplitudes and phases but also harmonic phase coupling information. 
Some of the conventional small-signal measurements for the pulse-compression NLTLs have been carried out at a laboratory of the Communications Research Centre (CRC), Ottawa. The same laboratory is contacting Agilent to review a possibility of purchasing an NVNA system. In this work, the large-signal testing was completed at the CRC laboratory, since an N5242A-type NVAN was provided by Agilent Technologies on a "demonstrator" basis.

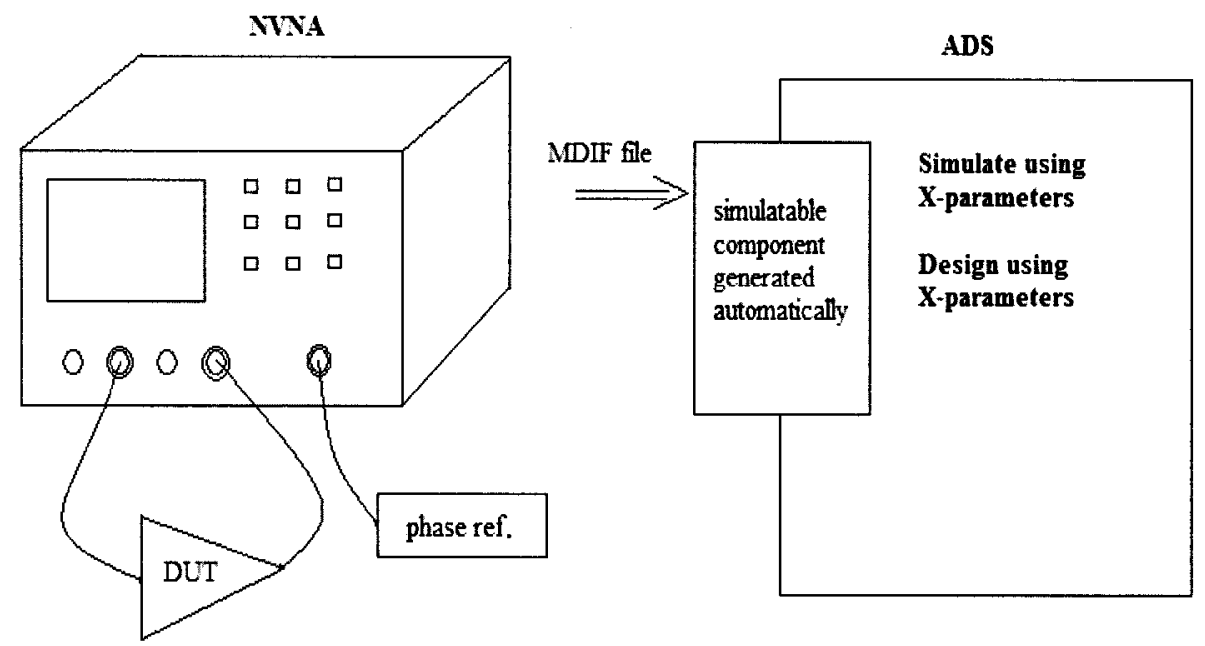

Figure 6-5 Simplified configuration of an NVNA and ADS workbench for nonlinear measurements.

\subsection{NLTL Chip Designs and Measurement Results}

In this work, two sets of NLTLs were designed. One set is an early design that neither used slow-wave CPW nor tapered-line techniques. The designed chip is shown in 
Figure 6-6. The chip size is $1.0 \times 2.0 \mathrm{~mm}^{2}$, the process being a standard CMOS $0.18-\mu \mathrm{m}$ technology. The top snake-like line in Figure 6-6 is an SDB NLTL with a length of 4.02 $\mathrm{mm}$, which includes 35 sections. The bottom snake-like line is an SDF NLTL with a length of $3.45 \mathrm{~mm}$, which also has 35 sections.

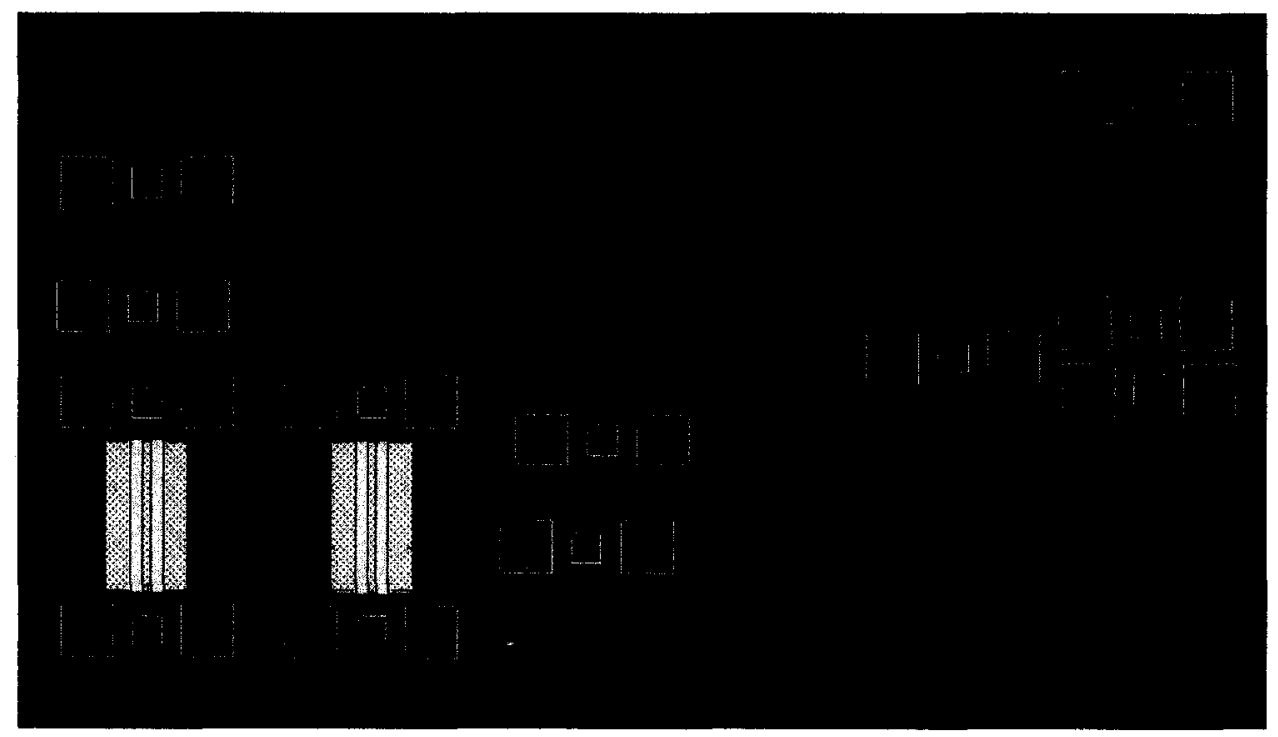

Figure 6-6 Early NLTL designs that omit slow-wave CPW and tapered-line techniques.

The latest NLTL chip design is shown in Figure 6-7. The chip size is $1.5 \times 2.0$ $\mathrm{mm}^{2}$, and the process is again a standard CMOS $0.18-\mu \mathrm{m}$ technology. The left-most line in Figure 6-7 is an SDB NLTL with a length of $1.06 \mathrm{~mm}$. The second-to-left line in the figure is an SDF NLTL with a length of $0.86 \mathrm{~mm}$. Both lines have 16 sections. Since both slow-wave CPW and tapered-line technologies are used, the NLTL lengths in the latest design are much shorter than in the earlier design. 


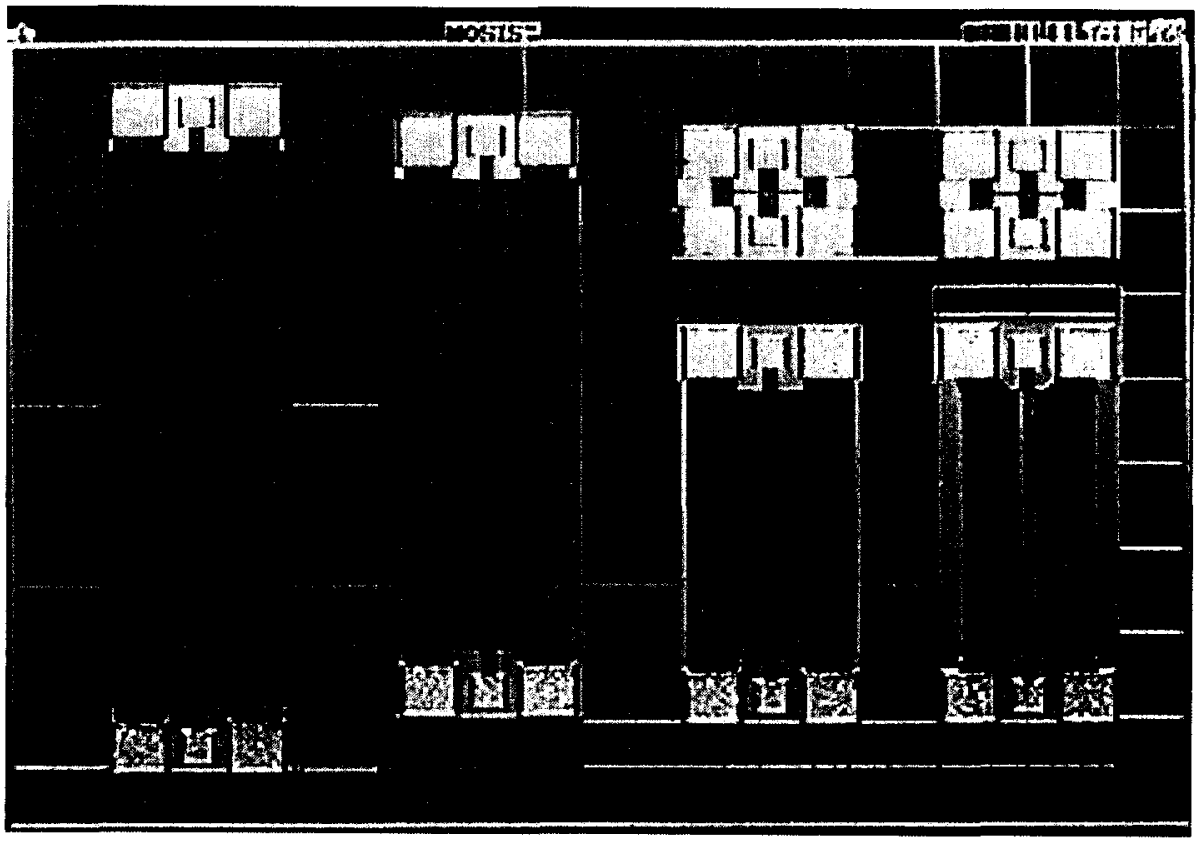

Figure 6-7 The latest NLTL designs, which use both slow-wave CPW and tapered-line technologies.

The large-signal NLTL measurements were done using an NVNA system provided by Agilent Technologies Inc. The maximum frequency capacity of the N5242A system was $26 \mathrm{GHz}$. The input signal was a 5-GHz sinusoidal wave since the pulse input (or square-wave input) feature is still under the development by the same company. This means that at the output of an NLTL, the highest harmonic we can see is the $5^{\text {th }}$ harmonic at $25 \mathrm{GHz}$. The maximum input available is $14 \mathrm{dBm}$. Since the equipment was available only on a "demonstrator" basis, the NLTLs were tested based on whatever features the equipment could provide. Some test results are shown below. In the figures for the NLTL time-domain measurement results, $\mathrm{A} 1$ indicates the input voltage wave, B2 the output voltage wave of the NLTLs, and B1 the reflected voltage wave. In the figures for the frequency-domain results, the markers, Mkr1, Mkr2, Mkr3,$\ldots$, identify the amplitudes of the $1^{\text {st }}, 2^{\text {nd }}, 3^{\text {rd }} \ldots$ harmonics. 
- Measurements on the most recent NLTL designs

Figures 6-8 to 6-15 give test results for the latest NLTL designs. Figure 6-8 shows time-domain results for the SBD NLTL with a $5-\mathrm{GHz}$ input at $13.0 \mathrm{dBm}$, which gives an input with 1.3 V-amplitude. Figure 6-9 gives frequency-domain results for the same device under the same conditions. The time-domain result shows that double-edge compression is happening and that the output B2 is squarer than the input. The frequency-domain result shows that more energy is accumulated in the higher harmonic components. For example, the fourth output harmonic is $41.7 \mathrm{dBm}$ higher than the fourth input harmonic, as shown in Figure 6-9. This is first test case.

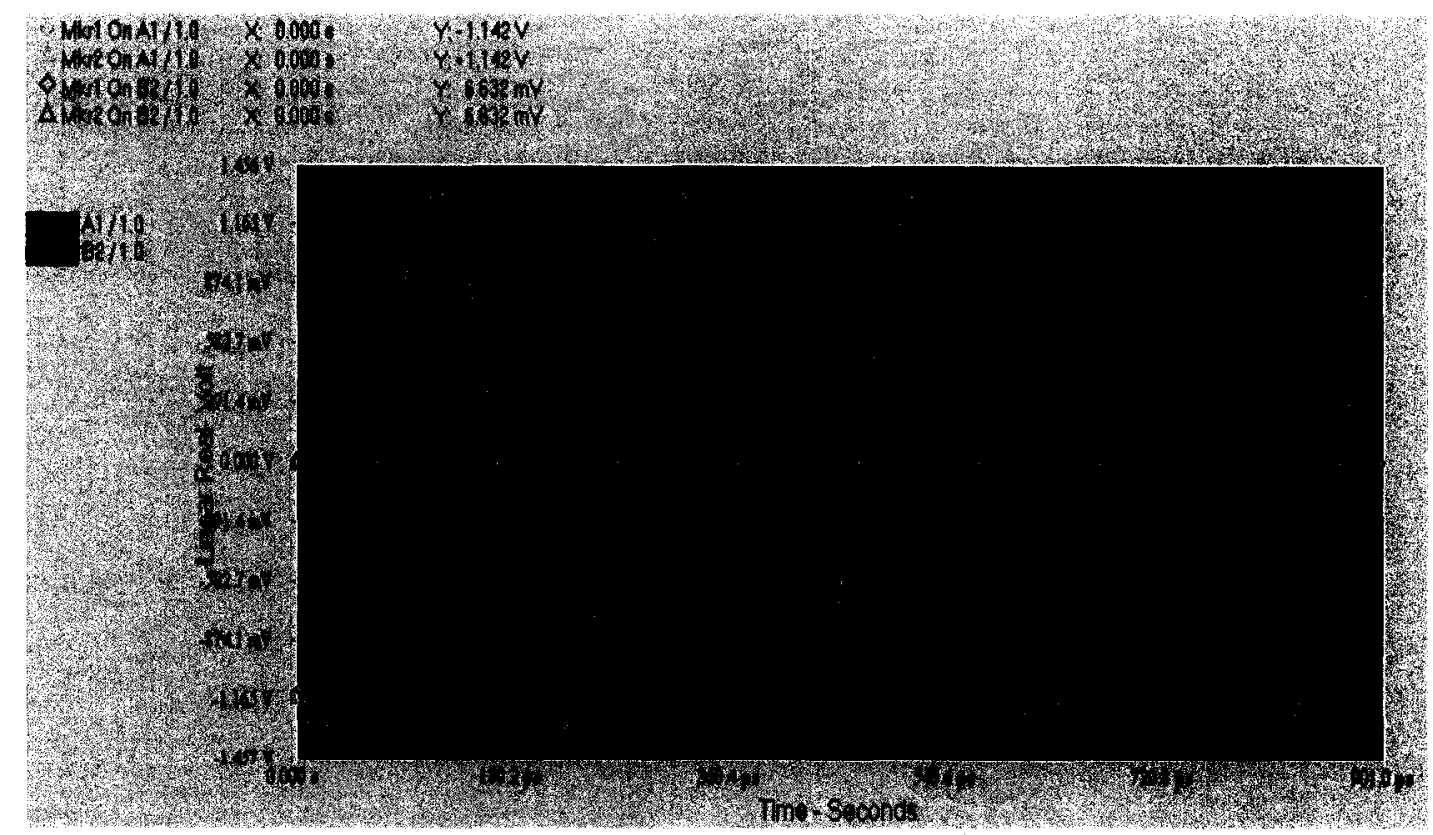

Figure 6-8 Measured SDB NLTL time-domain input (A1, yellow) and output (B2, blue) waveforms for a $13.0 \mathrm{dBm}$ input at $5 \mathrm{GHz}$. 


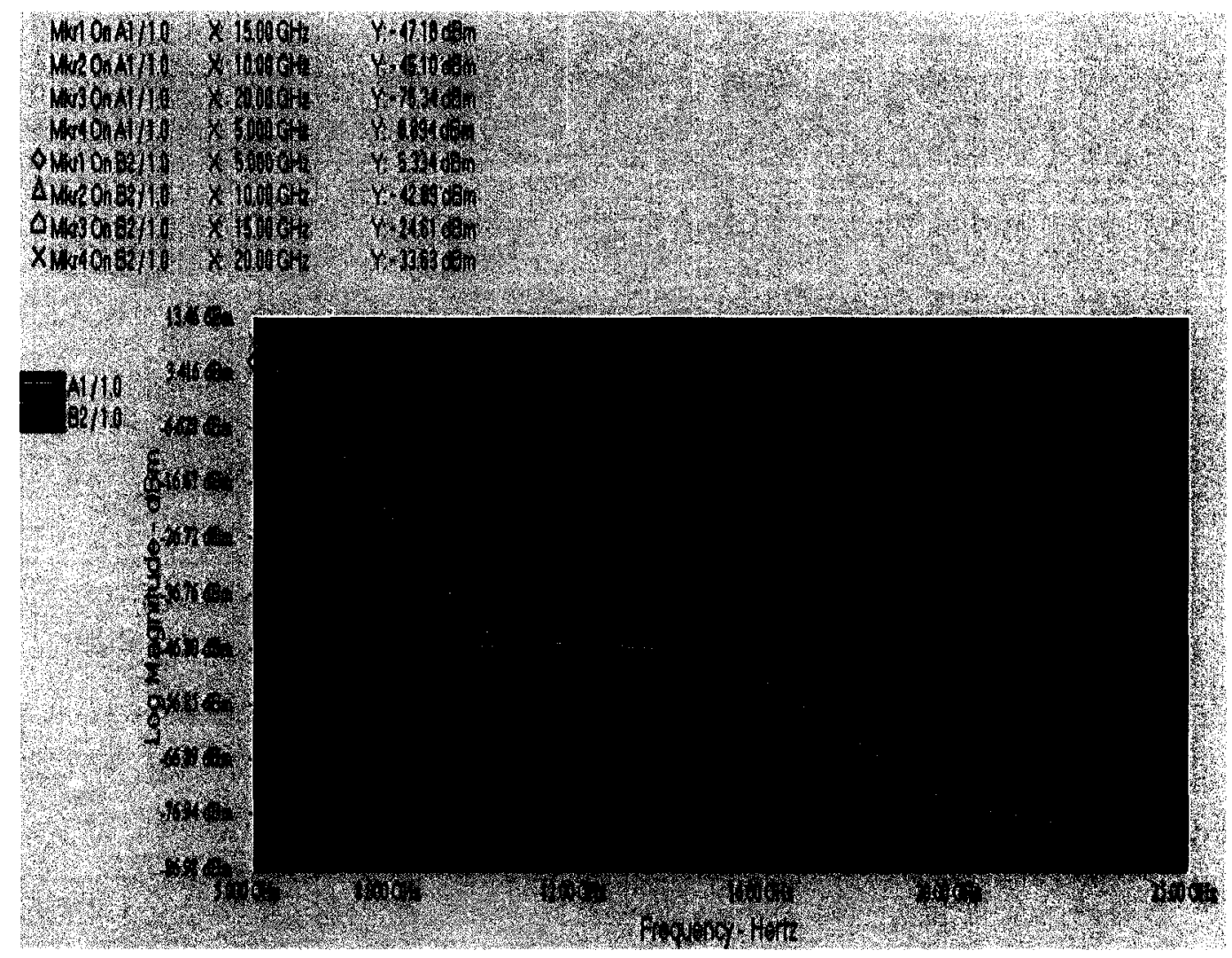

Figure 6-9 Measured frequency-domain SDB NLTL spectrum for a $13.0 \mathrm{dBm}$ input at $5 \mathrm{GHz}$.

The second test case is shown in Figures 6-10 and 6-11. It is for the SDB NLTL at $13.5 \mathrm{dBm}$ and $5 \mathrm{GHz}$ input, which gives an input with $1.35 \mathrm{~V}$-amplitude. The input power is slightly higher than in the first test case, and the double-edge compression effect is slightly stronger. The output is squarer than that of the first case. More important, the second output harmonic is $14 \mathrm{dBm}$ higher than the second input harmonic, as shown in Figure 6-11. This contrasts with in the first case, where the second output harmonic is only $2 \mathrm{dBm}$ higher than the second input harmonic, as seen in Figure 6-9. 


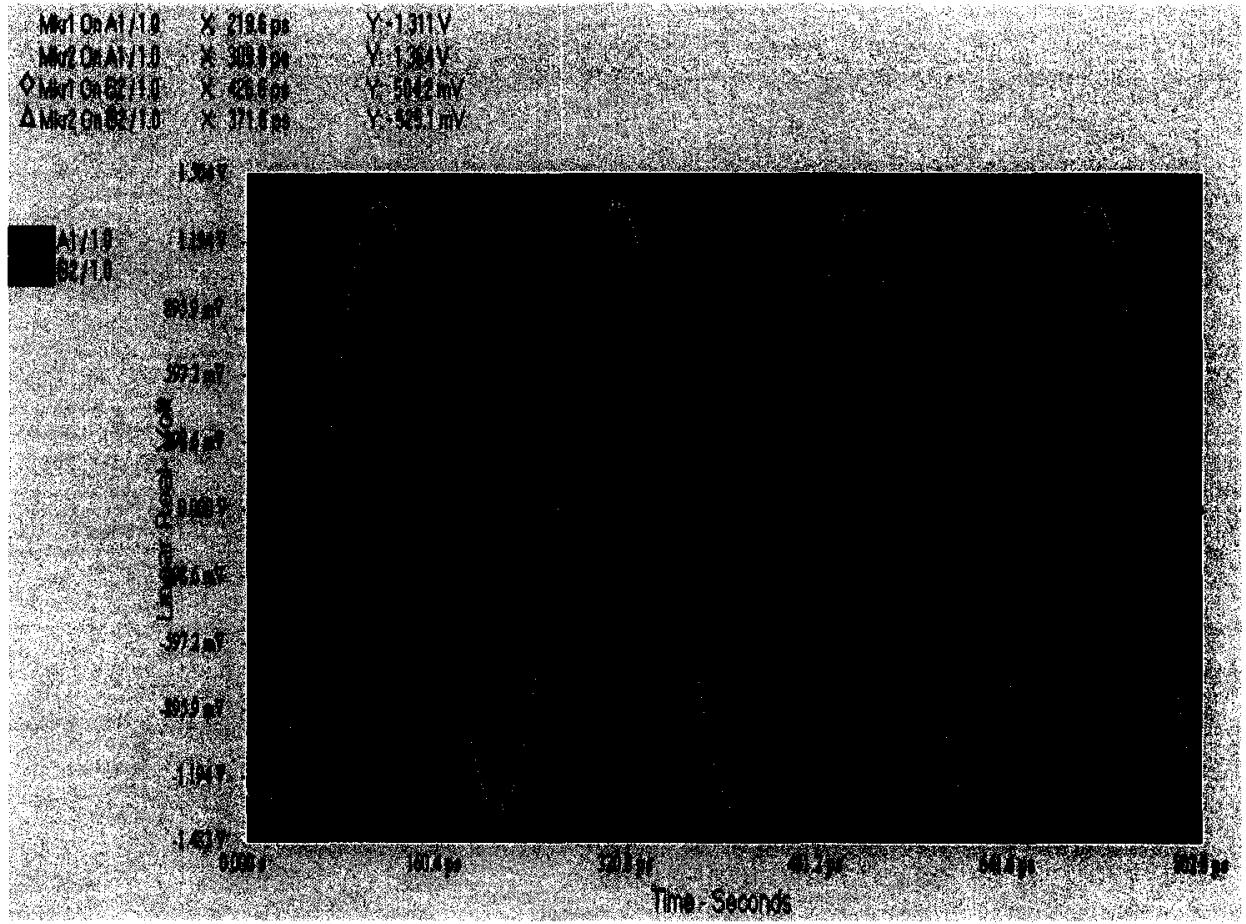

Figure 6-10 Measured SDB NLTL input (A1, yellow) and output (B2, blue) time-domain waveforms for a $13.5 \mathrm{dBm}$ input at $5 \mathrm{GHz}$.

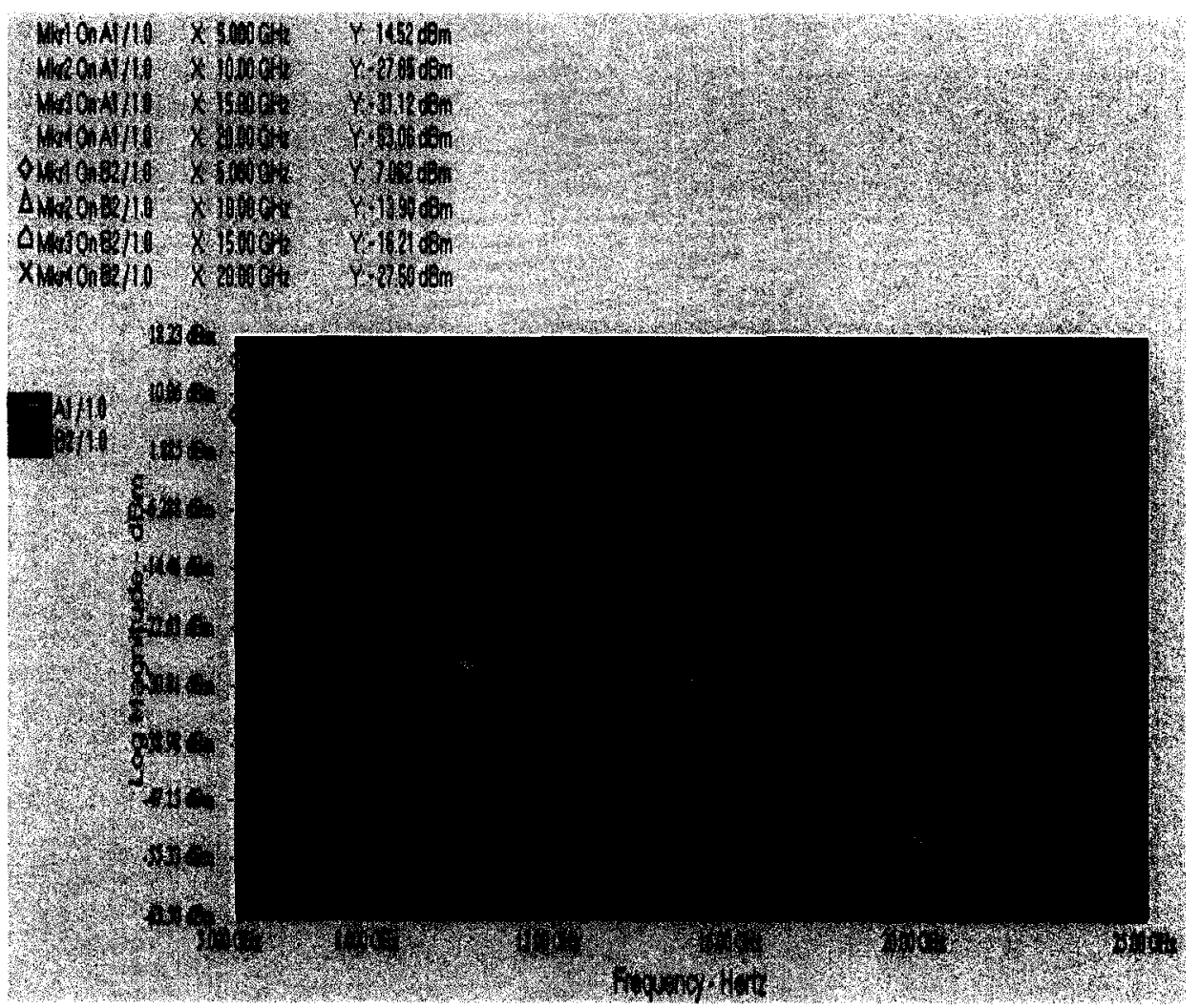

Figure 6-11 Measured frequency-domain SDB NLTL spectrum for a $13.5 \mathrm{dBm}$ input at $5 \mathrm{GHz}$. 
The third test case is also for the SDB NLTL but with a $5-\mathrm{GHz}$ at $13.9 \mathrm{dBm}$ input, which gives a 1.6 V-amplitude input. The results are shown in Figures 6-12 and 6-13. The NLTL performed a better double-edge pulse compression than the first two test cases. The output waveform better resembled a square shape than the two other cases. Compared to the input harmonics, the second output harmonic increases by $14 \mathrm{dBm}$, and the third one increases by $16 \mathrm{dBm}$, as shown in Figure 6-13. This case indicates that the higher the bias voltage, the stronger the compression. This may not always true. The reason is that the bias value determines the nonlinear capacitance value, which in turn decides the varactor cutoff frequency. The best compression happens only when the ratio of the Bragg frequency to the varactor cutoff frequency reaches certain values, as pointed out in Chapter 3.

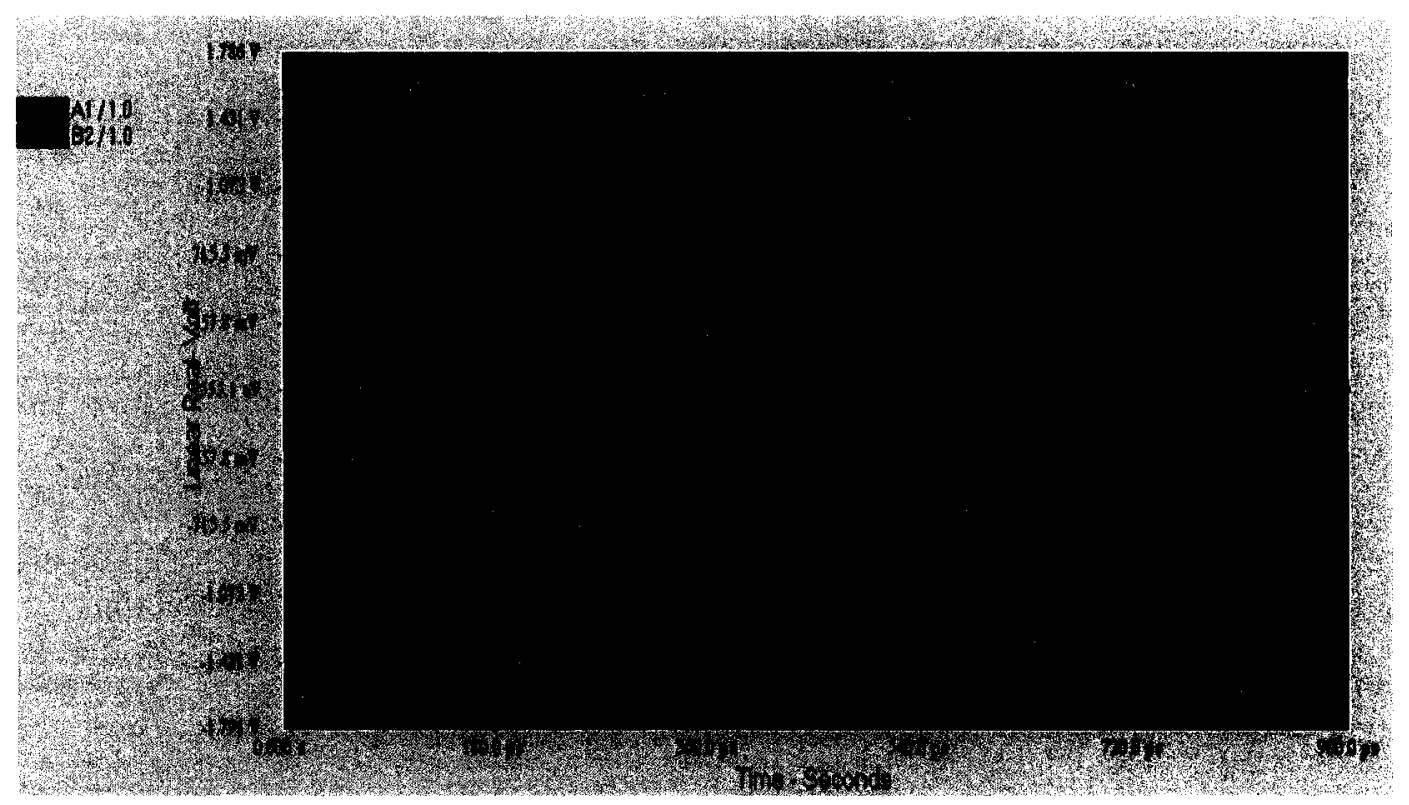

Figure 6-12 Measured SDB NLTL input (A1, yellow) and output (B2, blue) time-domain waveforms for a $13.9 \mathrm{dBm}$ input at $5 \mathrm{GHz}$. 


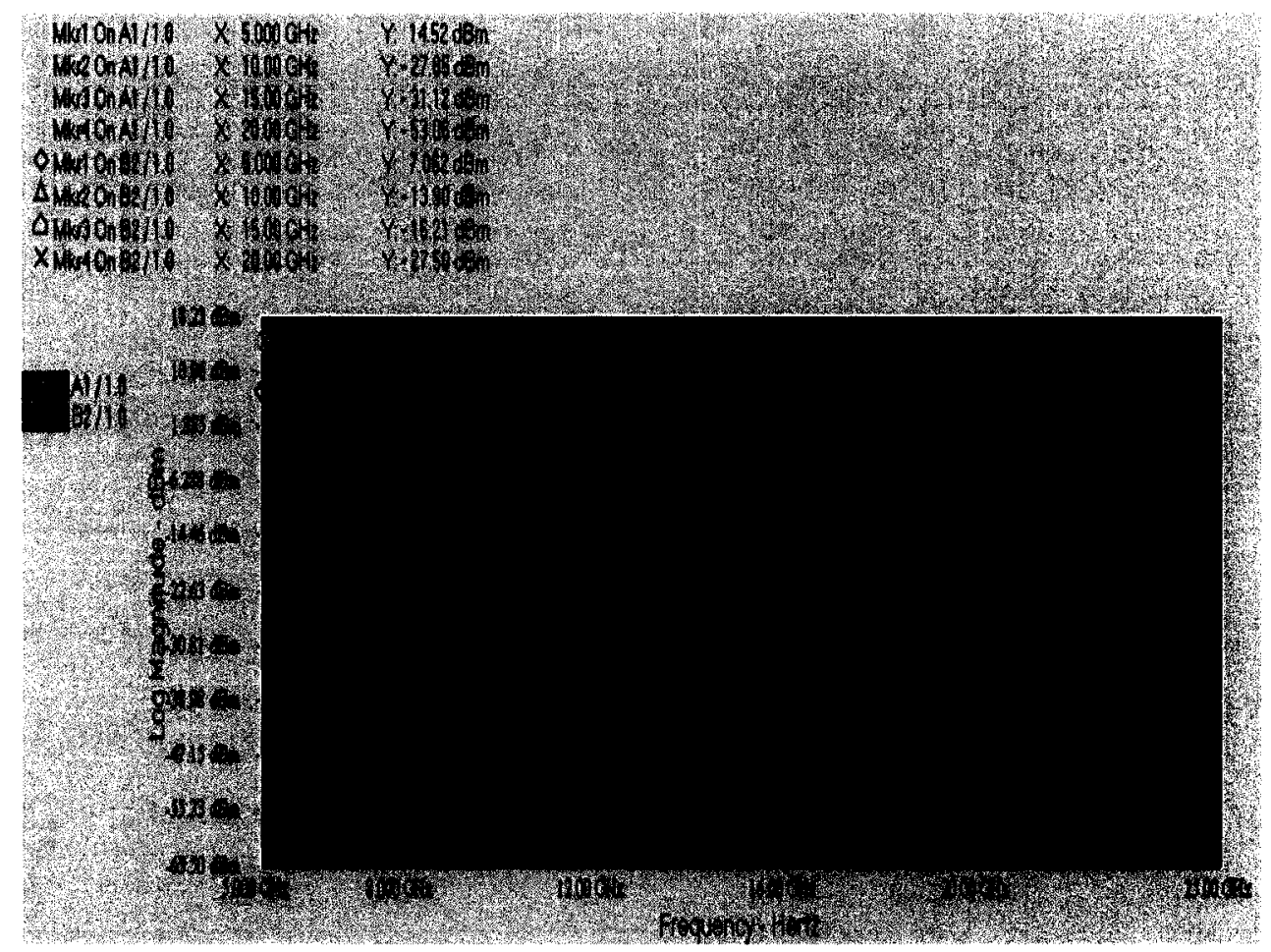

Figure 6-13 Measured frequency-domain SDB NLTL spectrum for a $13.9 \mathrm{dBm}$ input at $5 \mathrm{GHz}$.

Figure 6-14 and 6-15 are for the SDF NLTL. The input is a $3.0 \mathrm{GHz}$ sinusoidal wave at $13.0 \mathrm{dBm}$. Looking at time-domain result in Figure 6-14, a very minor compression on the rise-edge of the output B2 can be seen. The power level of the higher harmonics also has a slight change, the output second, third, fourth and fifth harmonics are all higher than the input relative ones, as shown in Figure 6-15. Other input settings have also been tried, but, in general, the single-edge compression is not very impressive. This kind of result for the SDF NLTL had been expected. As pointed out in Chapter 4, the SDF (or IMOS) varactor has a larger channel resistance than an AMOS varactor, since in the SDF structure the well makes no contribution to the channel conductivity. The larger channel 
resistance results in higher attenuation and the higher loss killed the nonlinearity. A better result might have been obtained if AMOS varactors had been used instead of SDF varactors.

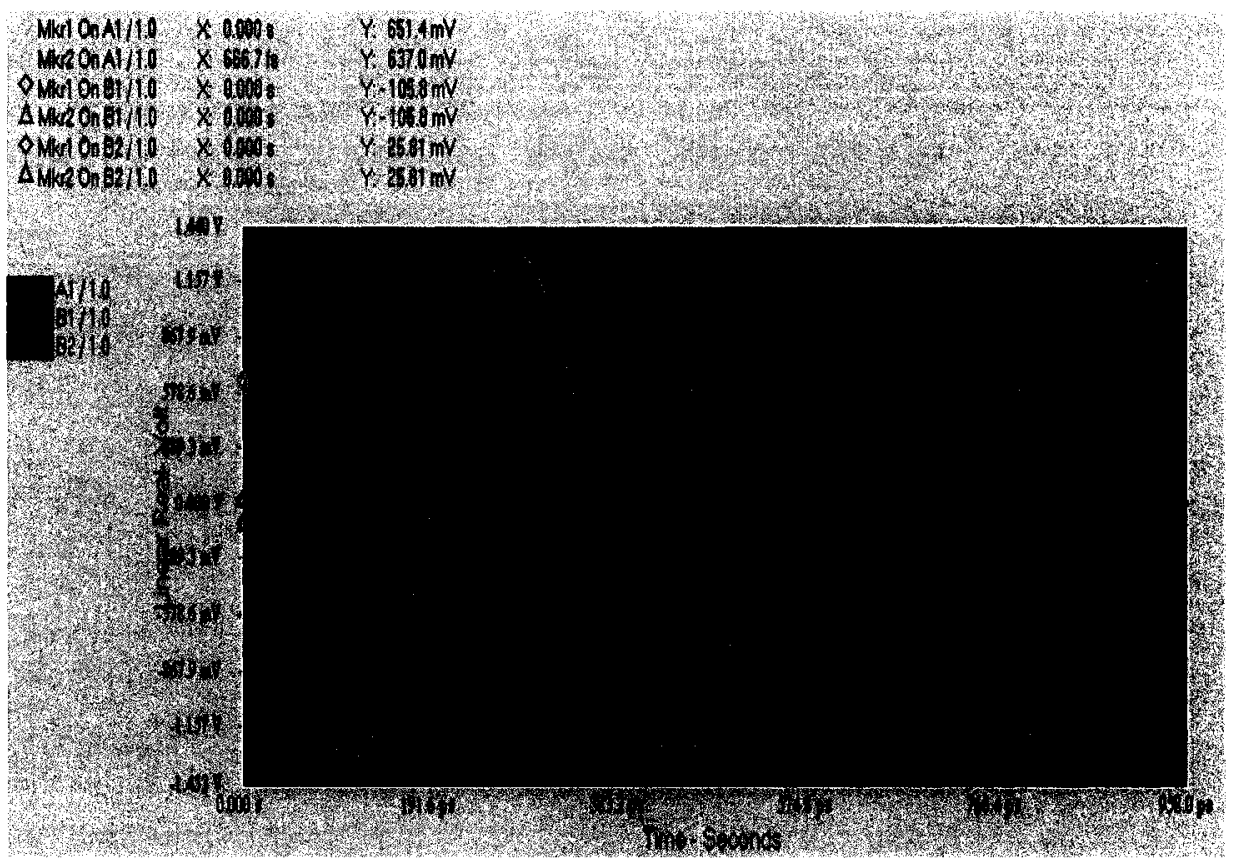

Figure 6-14 Measured SDF NLTL input (A1, yellow), reflected (B1, purple), and output (B2, blue) time-domain waveforms for a $13.0 \mathrm{dBm}$ input at $3 \mathrm{GHz}$. 


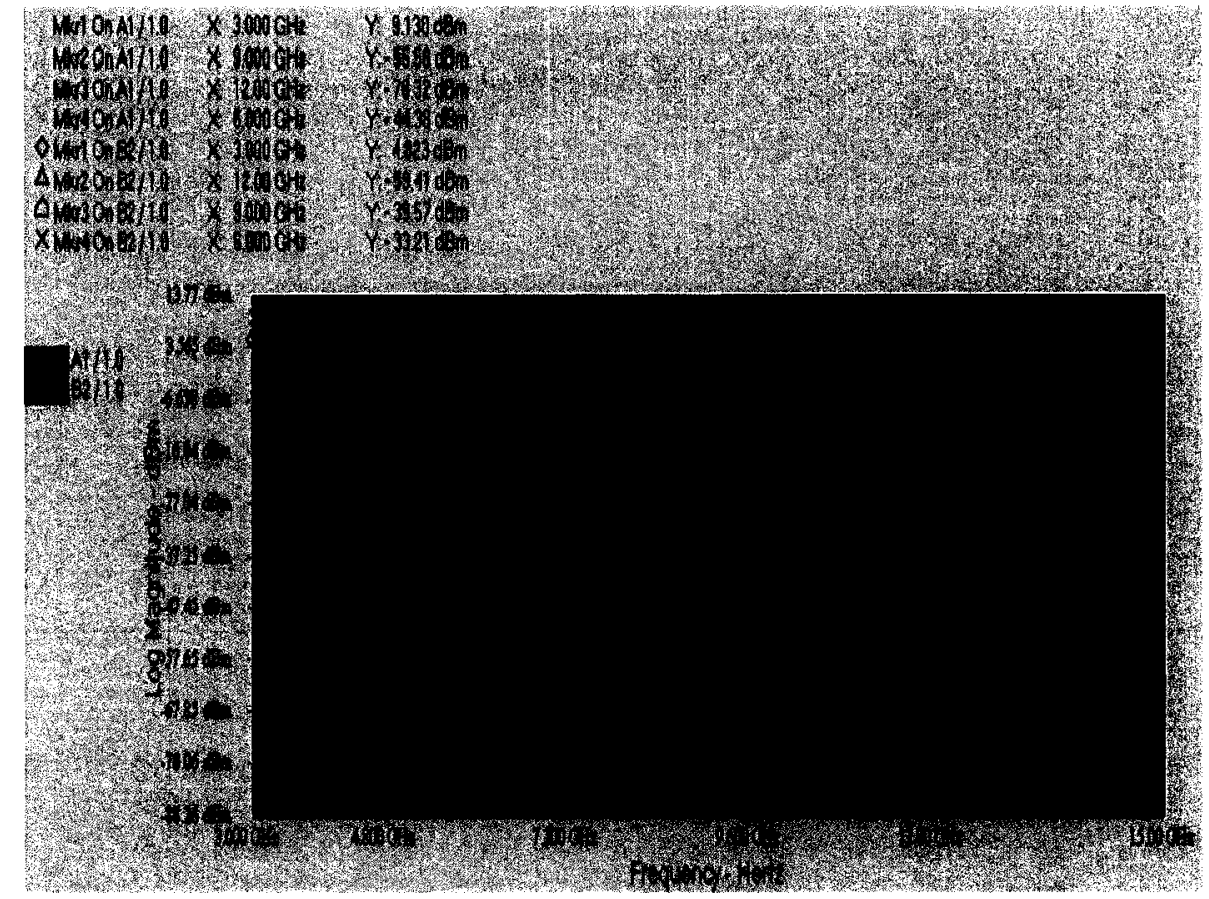

Figure 6-15 Measured frequency-domain SDF NLTL spectrum for a 13.0 dBm input at $3 \mathrm{GHz}$.

- Measurements on prototype NLTLs

Figure 6-16 and 6-17 are for the early SDF NLTL designs with input at $14.0 \mathrm{dBm}$ and 4.5 GHz. Nothing is really happening with this design since the device attenuation is too high. Similar results were obtained with other input settings and with the early SDB NLTL designs. 


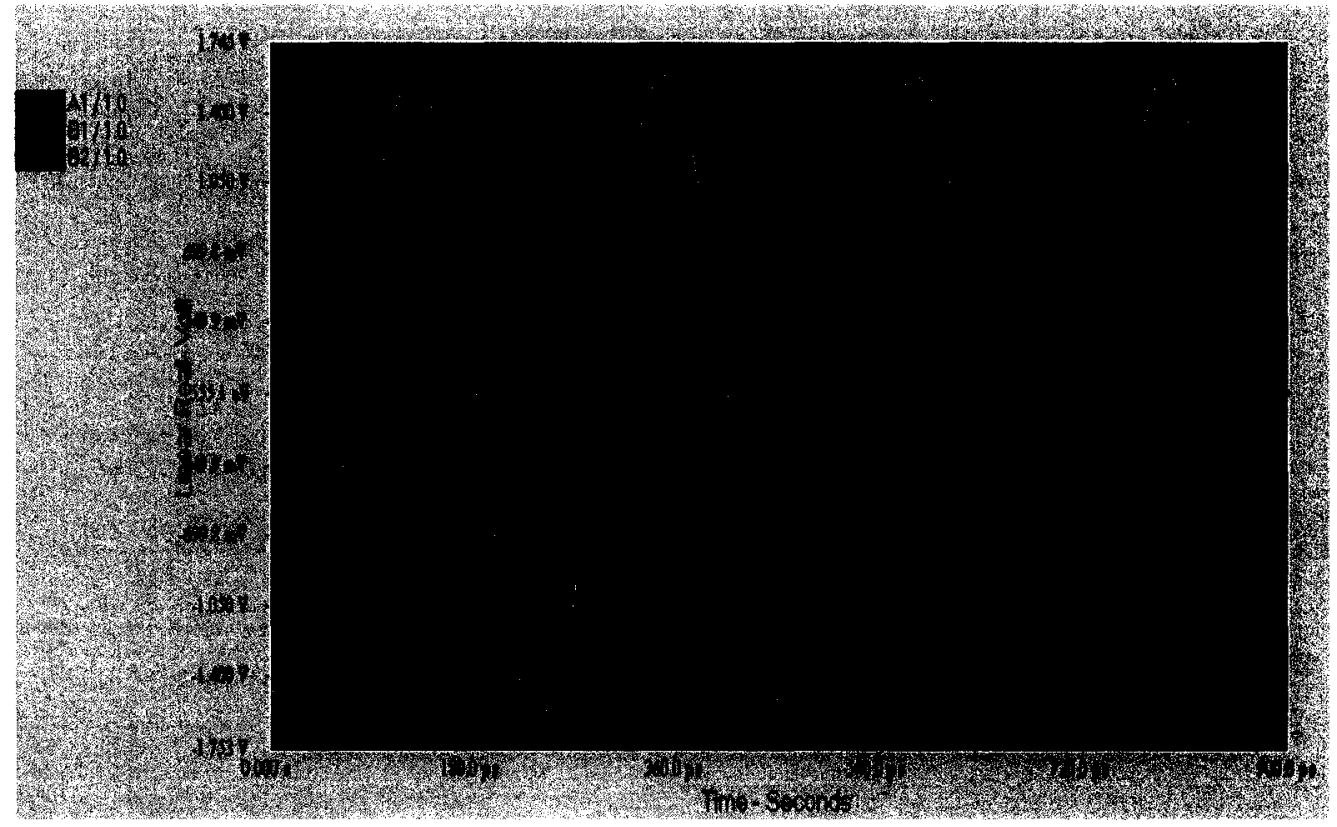

Figure 6-16 Measured SDF NLTL input (A1, yellow), reflected (B1, blue), and output (B2, purple) time-domain waveforms for a $14.0 \mathrm{dBm}$ input at $4.5 \mathrm{GHz}$.

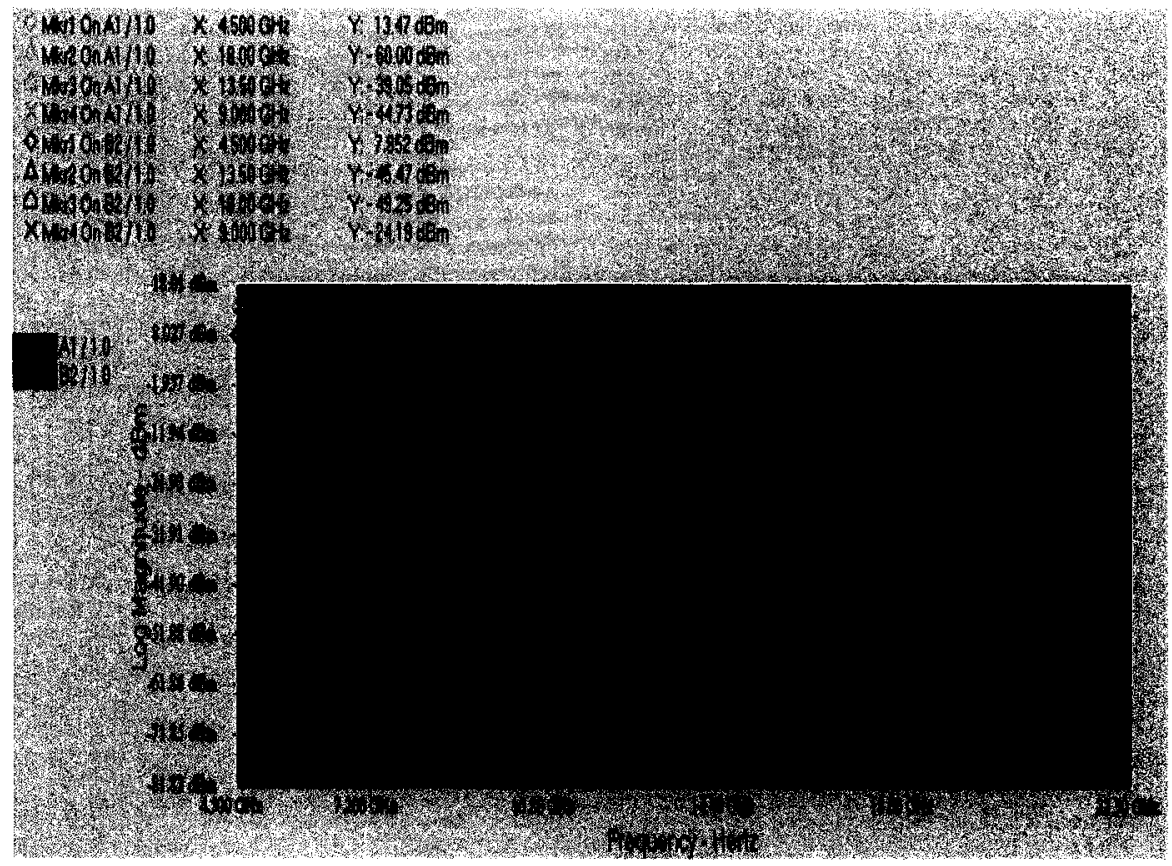

Figure 6-17 Measured frequency-domain SDF NLTL spectrum for a 14.0 dBm input at 4.5 GHz. 
To validate the large-signal test results, a few more extra simulations of SDB NLTLs were done using sinusoid-wave inputs instead of square-pulse-wave inputs. The same design data as that of the latest designed tapered slow-wave SDB NLTL were used in the simulations. With the frequency parameter set to $5 \mathrm{GH}$ for all three cases, Figures 6-18, 6-19 and 6-20 show the results with the input sinusoid wave amplitude parameter set to $2.2,2.3$ and $1.9 \mathrm{~V}$, respectively.

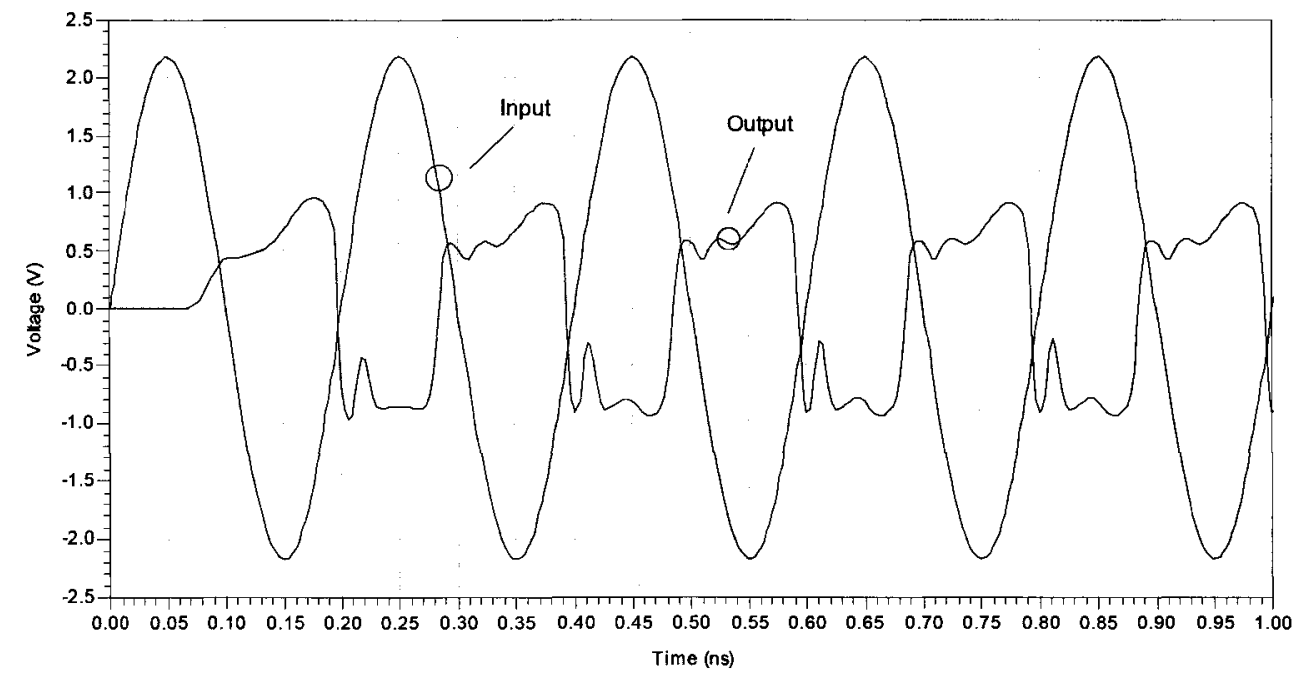

Figure 6-18 The SDB NLTL simulated result with $5 \mathrm{GHz}$ sinusoid input, $2.2 \mathrm{~V}$ amplitude. 


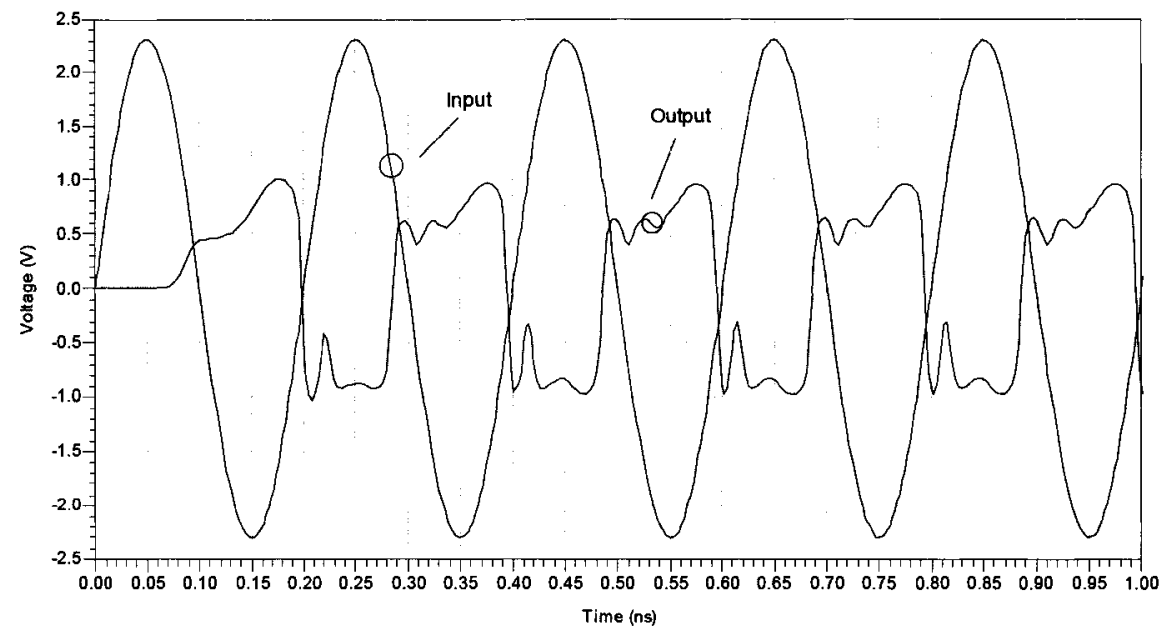

Figure 6-19 The SDB NLTL simulated result with $5 \mathrm{GHz}$ sinusoid input, $2.3 \mathrm{~V}$ amplitude.

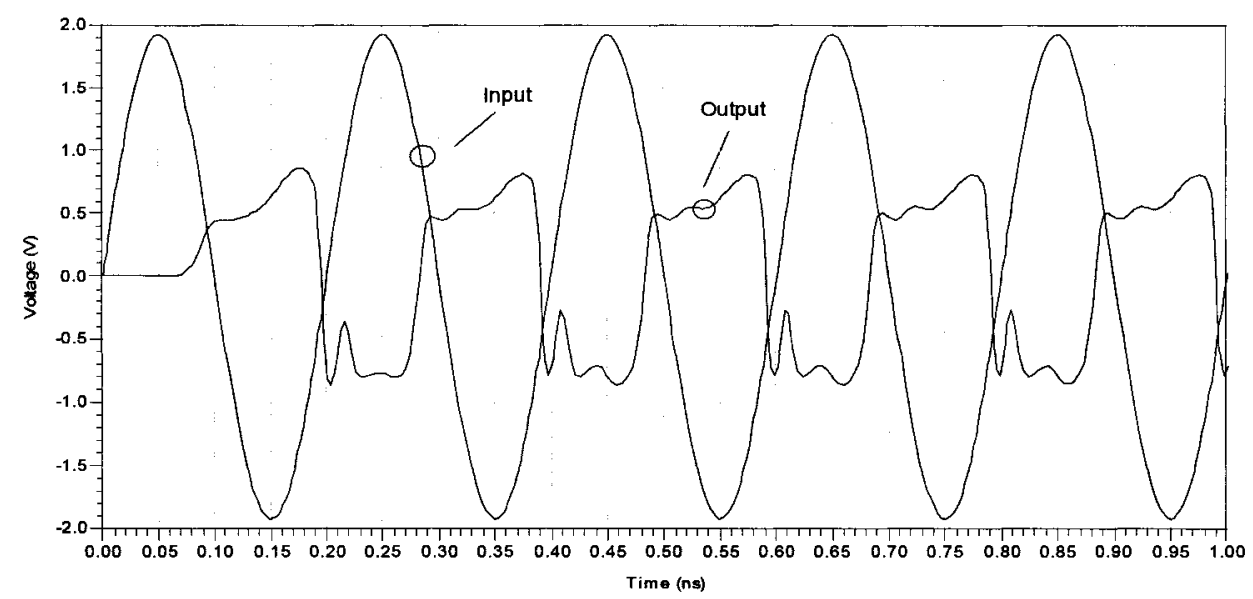

Figure 6-20 The SDB NLTL simulated result with $5 \mathrm{GHz}$ sinusoid input, $1.9 \mathrm{~V}$ amplitude.

Figures 6-18, 6-19 and 6-20 show that strong double-edge compression happens at the $1.9-2.3 \mathrm{~V}$ input level. Since there was a limitation on input power $(<14 \mathrm{dBm})$ 
during the large-signal testing, to match the test case, the amplitude of the sinusoid input in the simulation was reduced to $1.6 \mathrm{~V}$. The simulated result is shown in Figure 6-21. The double-edge compression is still effective but not as strong as that with the higher input power.

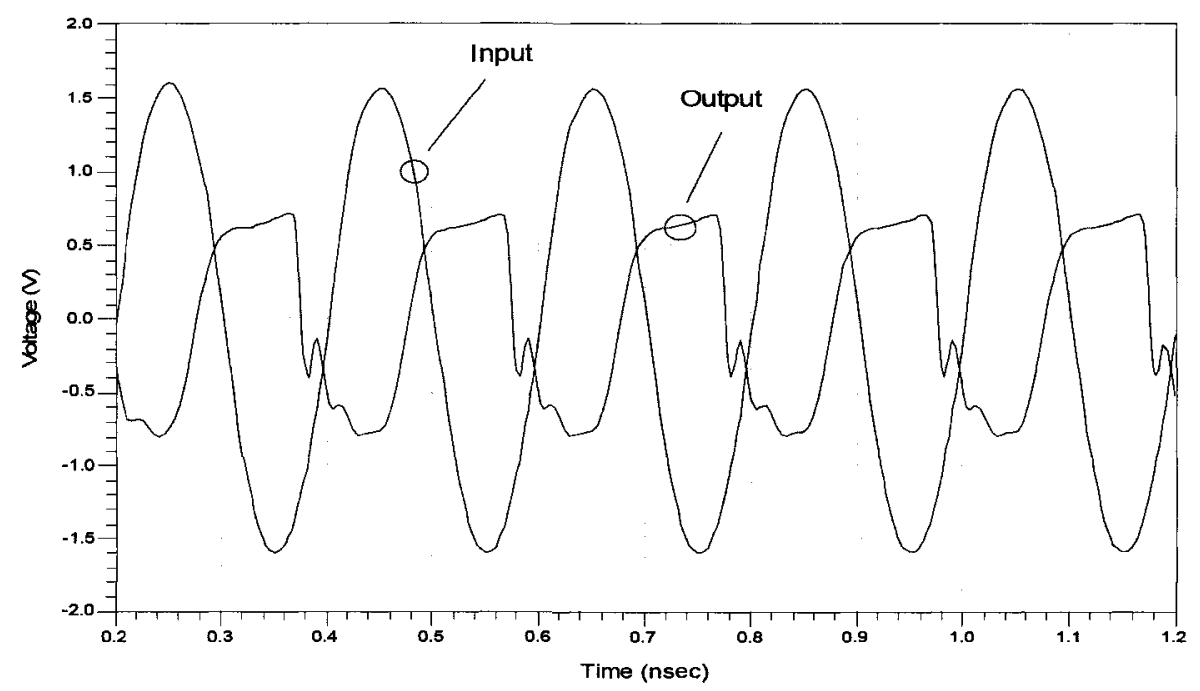

Figure 6-21 The SDB NLTL simulated result with $5 \mathrm{GHz}$ sinusoid input, $1.6 \mathrm{~V}$ amplitude.

Figure 6-22 is a re-constructed waveform of the output signal in Figure 6-21 by using only the 5 lowest output harmonics. With $5 \mathrm{GHz}$ input, the highest harmonic the large-signal testing instrument NVNA can cover is the $5^{\text {th }}$. This part of the work demonstrates that the test results are reasonable, and that the SDB NLTL is indeed compressing the input signal. Figure 6-23 shows the spectrum of the input signal and the 5 lowest output harmonics. The dot-dash line is for input signal and the solid line for output signal. One can see that the waveform in Figure 6-22 is very similar to the 
waveform in Figure 6-12, and the spectrum of the first five harmonics in Figure 6-23 is very similar to the spectrum in Figure 6-13.

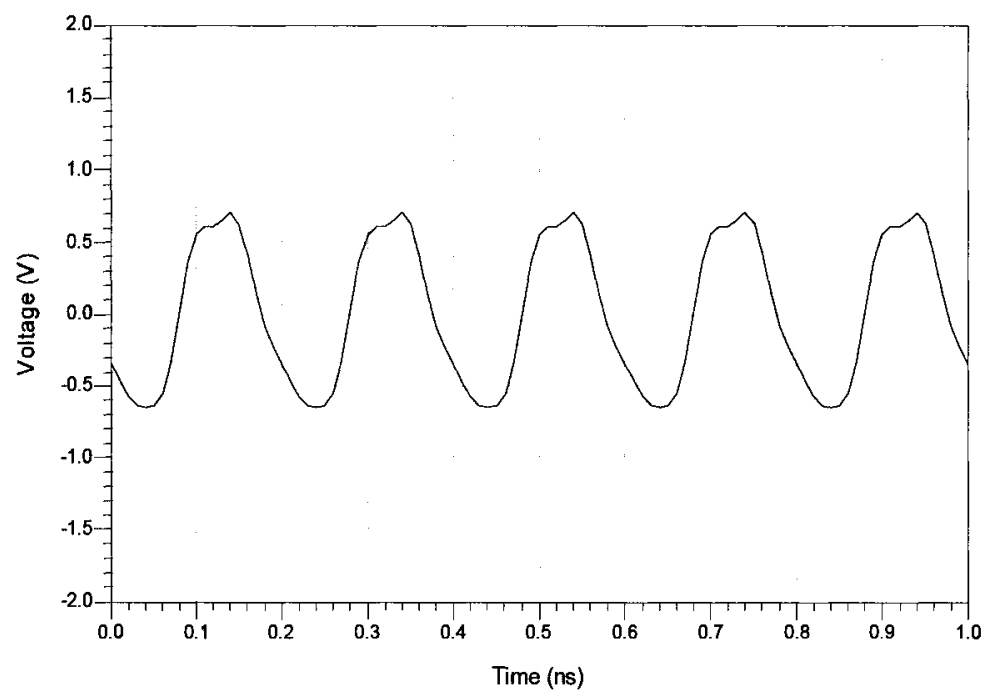

Figure 6-22 Reconstructed waveform by using only 5 lowest output harmonics.

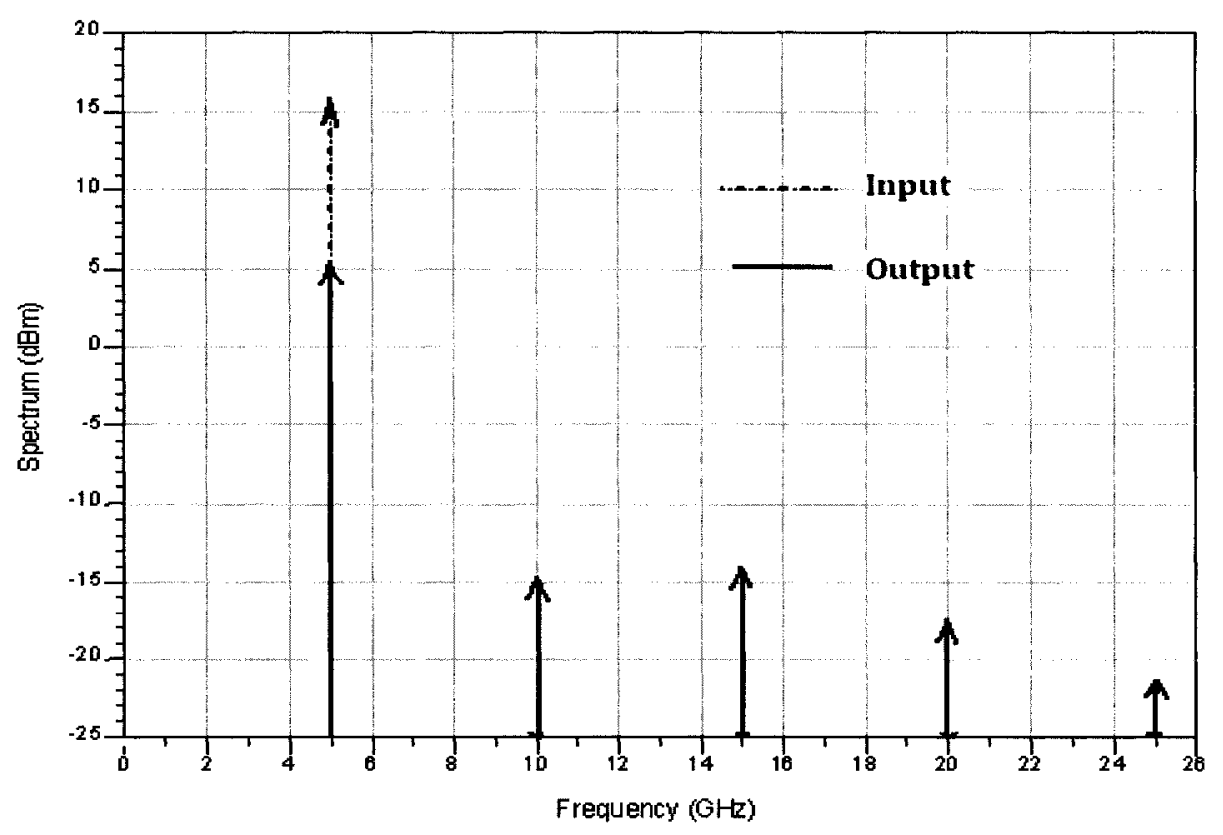

Figure 6-23 Spectrum of an input signal and 5 output harmonics. 


\subsection{Conclusion}

This chapter discussed both NLTL small-signal and large-signal measurement strategies. For the small-signal testing, both calibrating the testing equipment and deembedding of on-chip pad-parasitics are required. Different kinds of calibration and de-embedding technologies were presented, evaluated and compared. For the largesignal testing, a recently-developed harmonic-oriented technique equipped with nonlinear VNA was used. Instead of $S$-parameters, $X$-parameters were used to describe large-signal nonlinear behaviour. The theory of the $X$-parameter technique was presented, and the testing method was discussed in this chapter. A brief description of NLTL chip designs was also presented.

Finally, the NLTL large-signal testing results were presented. Simulations using sinusoidal inputs demonstrated that the large-signal testing was giving correct results.

This work strongly indicates that it should be possible to build excellent pulsecompression NLTLs using a low-resistivity commercial CMOS process. 


\section{Chapter 7 Summary and Future Directions}

This work investigates the design of all-silicon pulse-compression NLTLs. A slow-wave CPW technology was developed to reduce the silicon loss. Two types of NLTLs were introduced. The SDF type, optimized for single-edge pulse sharpening, is able to reduce the rise time by $75 \%$, while the SDB type, suited for double-edge pulse sharpening, is able to reduce both rise and fall times by $60 \%$. The simulations are based on measured data from on-chip CPW and varactor components of the NLTLs. Five CMOS chips were developed and fabricated in this study. Some of them are for CMOS varactor research, some for silicon CPW research, and some for research on de-embedding techniques. More importantly, two sets of pulse-compression NLTLs were developed on the chips. Each set includes two NLTL lines, one for single-edge pulse compression, and one for double-edge pulse compression. One of these two sets of NLTLs was designed in the early stages of the study; in this case, both NLTL lines were more than $3 \mathrm{~mm}$ long since they were designed using neither tapered-line nor slow-wave technologies. The last two designs are much better, since their lengths are both shorter than $1 \mathrm{~mm}$. This is because both tapered-line and slow-wave techniques were used. The large-signal behaviour of both sets of NLTLs was investigated and measured using nonlinear VNA instrumentation. Since the new nonlinear VNA instrumentation was still under development, the measurements were restricted to sinusoidal inputs and output frequencies up to only $26 \mathrm{GHz}$. That is, with $5 \mathrm{GHz}$ input, only up to 5 harmonics could be measured. Even through the testing instrument had limitations, the testing results are still very promising. The most recently designed 
double-edge compression NLTL showed a strong 'squaring' effect on an input sinusoidal wave. Almost certainly, better results could have been obtained if the higher harmonics could have been measured. The most recently designed single-edge compression NLTL showed very little sign of compression, due to its larger attenuation. Since this kind of NLTL uses the SDF varactor structure, in which the source and drain of the transistor are floating from the bulk, the well makes no contribution to the channel conductivity, resulting in a larger channel resistance. The large attenuation of the SDF structure decreases the nonlinearity in the NLTL circuits. The prototype NLTLs showed hardly any compression due to their longer length with larger attenuation, as was expected.

This work shows the feasibility of building all-silicon pulse-compression NLTLs using commercial silicon foundry processes. This study has built up rich experience and knowledge on the design of low-loss low-cost pulse-compression NLTLs.

This research work has already produced the following publications:

[1] Ming Li, Rony E. Amaya, Jean-Marc Duchamp, Philippe Ferrari, Robert G. Harrison, and N. Garry Tarr, "Low-Loss Low-Cost All-Silicon CMOS NLTLs for Pulse Compression," IEEE MTT-S International Microwave Symposium, pp. 449-452, Honolulu, Hawaii, June, 2007. 
[2] Ming Li, Robert G. Harrison, Rony E. Amaya, Jean-Marc Duchamp, Philippe Ferrari, and N. Garry Tarr, "CMOS Varactors in NLTL Pulse-Compression Applications," Proceedings of the 37th European Microwave Conference, pp. 14051408, Munich, Germany, Oct., 2007.

[3] Rony E. Amaya, Ming Li, Robert G. Harrison, and N. Garry Tarr, "Coplanar Waveguides in Silicon with Low Attenuation and Slow Wave Reduction," Proceedings of the 37th European Microwave Conference, pp. 508-511, Munich, Germany, Oct., 2007.

[4] Ming Li, Rony E. Amaya, Robert G. Harrison, and N. Garry Tarr, "Investigation of CMOS Varactors for High-GHz-Range Applications," Accepted by the journal Research Letters in Electronics, May 2009.

Additional research publications are expected as direct results of this work, especially on the nonlinear results of large-signal measurements.

Further research work following this study could include:

1. Analytical work on the slow-wave propagation properties of all-silicon NLTLS

The present study has shown that the slow-wave CPW technique has better conversion efficiency at higher frequencies. This is because that the floating 
ground in the slow-wave structure can reduce the substrate loss, which is important because the substrate loss is proportional to the operating frequencies. It will be very interesting and meaningful to investigate the slow-wave CPW behaviour at higher frequencies, such as over $100 \mathrm{GHz}$. Another beneficial study on slow-wave CPWs would focus on their secondary Bragg frequencies. Since the floating ground introduces a secondary periodic structure into the system, there will be a secondary Bragg frequency. This secondary Bragg frequency is higher than the primary Bragg frequency, and may have an impact on the dispersion and primary Bragg frequency of an NLTL. It is anticipated that further study will discover the relationship among these propagation properties.

2. Developing new NLTL structures using AMOS and short-gate SnDp varactors

The study has shown that the AMOS varactor is better than the IMOS (SDF) varactor for single-edge pulse compression because of its reduced attenuation and its lower degree of $C(V)$-degeneration at high frequencies. For the same reasons, the short-gate $\mathrm{SnDp}$ varactor is better than NMOS (SDB) varactor for doubleedge pulse compression. The SDF and SDB varactors were used in the NLTL designs in this study because they were available components in the CMOS process. With the fast-developing pace of CMOS technology, it will be possible in the near future to build AMOS and SnDp NLTLs, and it is expected that they will provide better pulse-compression capabilities. 


\section{Appendix A: Propagation Characteristies of CPW Lines}

According to [39] and [42], without making any quasi-static assumptions, when a signal represented by

$$
v(t, z)=e^{-\alpha z} e^{j(\omega t-\beta z)}
$$

propagates through the CPW shown in Figure A-1, the phase constant $\beta$ is given by (A2).

$$
\beta(f)=2 \pi \cdot \frac{f}{c} \cdot \sqrt{\varepsilon_{e f f}(f)}
$$

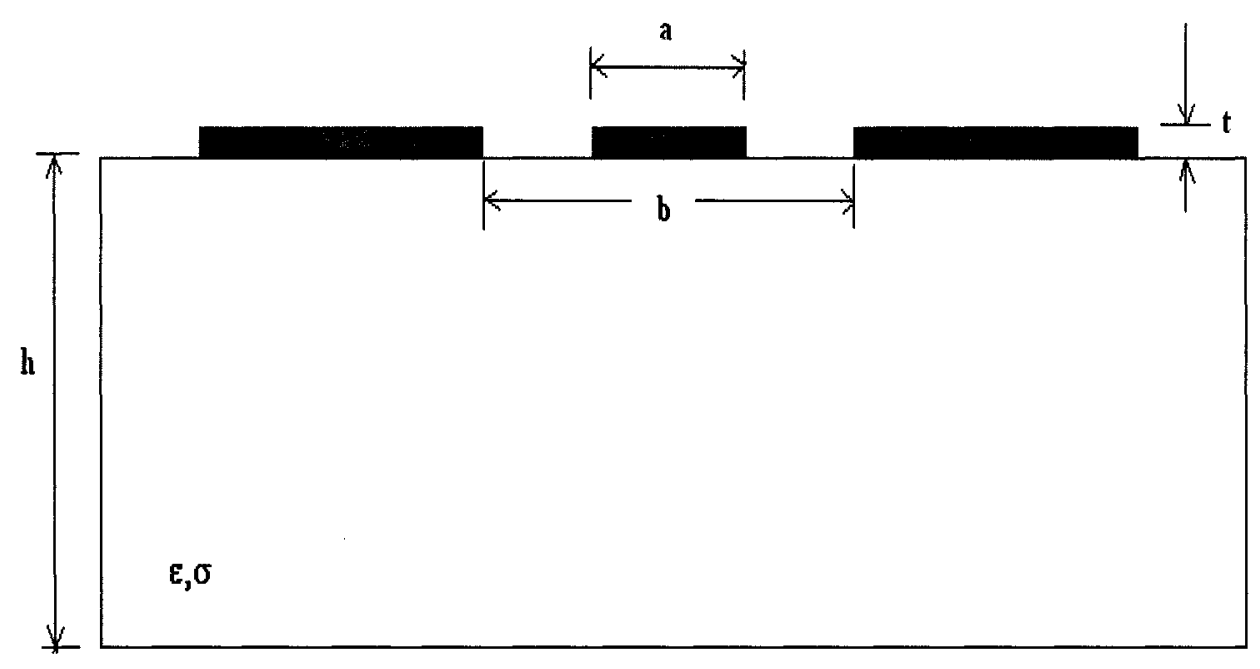

Figure A-1 Cross-section view of a CPW. 
In (A-1) $\alpha$ is the attenuation and in (A-2) $c$ is the vacuum speed of light.

Zero dispersion occurs when the phase constant $\beta$ is a linear function of frequency. The phase constant is a function of the effective permittivity $\varepsilon_{e f f}$. If $\varepsilon_{e f f}$ varies with the frequency, there is dispersion in the CPW line. For this structure, $\varepsilon_{\text {eff }}$ is given by (A-3) [42]

$$
\sqrt{\varepsilon_{e f f}(f)}=\sqrt{\varepsilon_{q}}+\frac{\varepsilon_{r}-\varepsilon_{q}}{1-n \cdot\left(\frac{f}{f_{t e}}\right)^{-b}},
$$

where $b=1.8, \varepsilon_{r}$ is the relative permittivity of the substrate, $\varepsilon_{q}=\frac{\varepsilon_{r}+1}{2}$ is the quasistatic effective permittivity, and $f_{t e}=\frac{c}{4 h \sqrt{\varepsilon_{r}-1}}$ is the surface wave $\mathrm{TE}_{10}$-mode cutoff frequency. The factor $n$ can be computed from [42]

$$
\begin{aligned}
& \log (n)=u \cdot \log (2 a /(b-a))+v \\
& u=0.54-0.64 \cdot q l+0.015 \cdot q l^{2} \\
& v=0.43-0.86 \cdot q l+0.54 \cdot q l^{2} \\
& q l=\log (a / h)
\end{aligned}
$$


where $a, b$, an $h$ are defined in Figure A-1.

The characteristic impedance is given by

$$
Z_{c p w}=\frac{30 \pi}{\sqrt{\varepsilon_{e f f}}} \cdot \frac{K\left(k^{\prime}\right)}{K(k)}
$$

where $K(k)$ is the complete elliptic integral of the first kind, with $k=a / b$ and $k^{\prime}=\sqrt{1-k^{2}}$. 


\section{Appendix B: Calibration Methods for CMOS small-signal measurements}

\section{(1) Three On-wafer Calibration Methods}

The three commonly used on-wafer calibration methods are the "Through-ReflectLine" (TRL), “Through-Reflect-Match" (TRM), and "Short-Open-Load-Through (SOLT) techniques. They can be used with on-wafer coplanar-waveguide standards to calibrate measurement systems up to the probe tips. Figure B-1 shows the structures of these three calibration standards [69].

Here is brief summary of the three methods [75]:

- The TRL method

The TRL method uses multiple transmission lines as standards. These transmission lines are built on the same wafer as the DUT. The measured $S$ parameters use the $T R L$ line $Z_{0}$ as the reference impedance. After calibration, the reference plane is precisely set at the center of the 'Thru' line. But this method requires multiple probe spacings, depending on the line impedances, and is therefore not suitable for fixed-spacing probes. The TRL method is a frequency-dependent method. If one needs to extend the testing frequency range, multiple lines have to be put on the chip, which takes more chip space. 


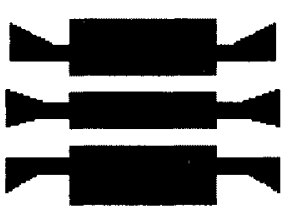

Thru

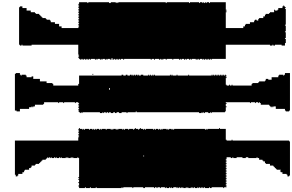

Thru

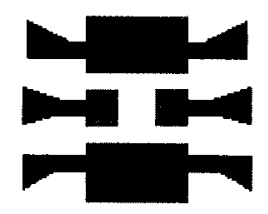

Reflect

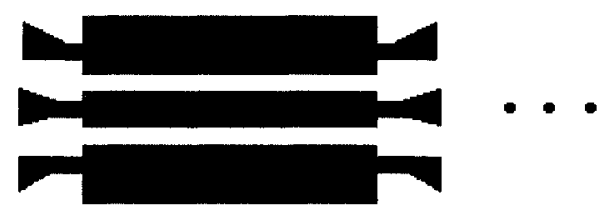

Line(s)

(a)

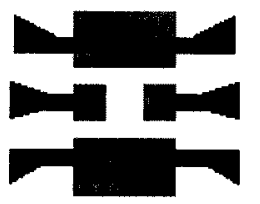

Reflect

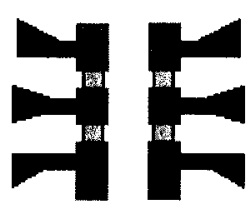

Match

(b)

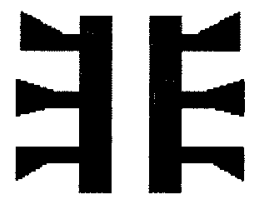

Short

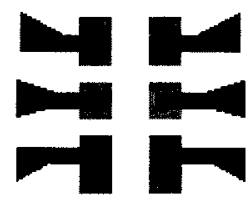

Open

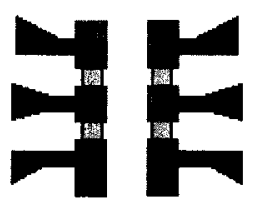

Load

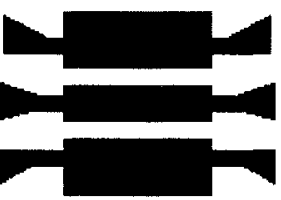

Thun

(c)

Figure B-1 Three calibration structures on coplanar waveguide standards, (a) TRL, (b) TRM, and (c) SOLT.

- The TRM Method 
Different from $T R L$ method, the $T R M$ method is a frequency-independent method. It is good for broadband calibrations and measurements. This method uses the matching resistance $Z_{\text {match }}$ of the TRM 'Match' structure as the reference impedance. To have a precise measurement, two match standards, one for each DUT port, are required. The TRM method is not suitable for CMOS chip, since process variations make it difficult to build two identical match standards. Besides the $S$-parameters of the TRM standards, the 'Thru' line delay should also be known for the calibration. Fixed probe spacings can be used with the $T R M$ calibration.

- The SOLT Method

The SOLT method is the most popular method and available on virtually every vector network analyzer. This calibration method performs reasonably well as long as accurate models of calibration standards are determined. The SOLT standards take less chip space than TRL and TRM methods, and can be used for broadband measurements. The negative part of this method is that the measurement results are very sensitive to probe placement. Bad probe positions can introduce negative capacitance to the measurement of the 'Open' standard, extra inductance to the measurements of the 'Short' and 'Load' standards, and cause unpredictable measured results. 
The calibration algorithms are available in references [63], [76], [77].

\section{(2) Pad Parasitic De-Embedding Methods}

(a) Vandamme's three-step de-embedding Method

This de-embedding algorithm is based on an equivalent circuit obtained from RF measurements. The equivalent circuit is shown in Figure B-2 (a). Figure B-2 (b) gives four de-embedding structures which need to be built on the chip together with the device under test (DUT).

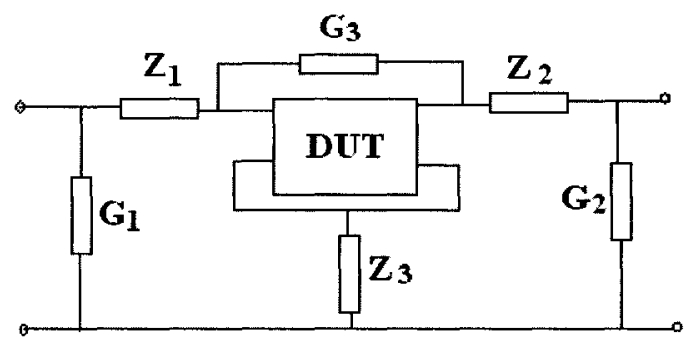

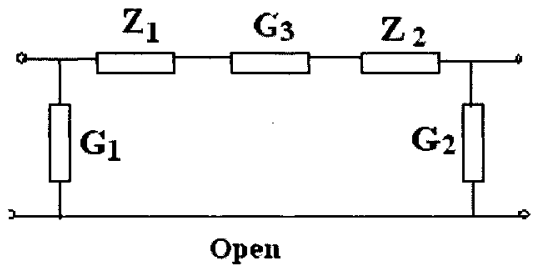

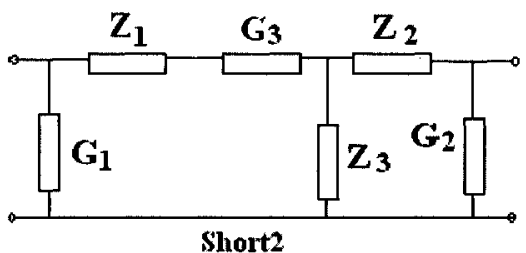

(a)
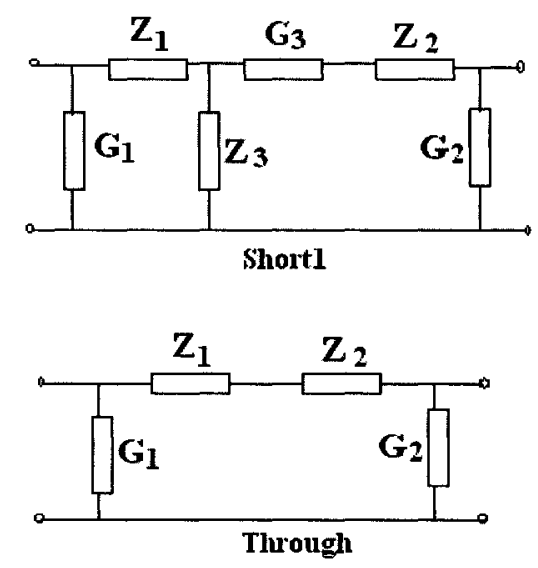

(b)

Figure B-2 (a) Equivalent circuit of the RF test structure. (b) Equivalent circuits of the open, short1, short 2 , and through de-embedding structures. 
Measuring the four de-embedding structures give four sets of $S$-parameters which are used to determine the six unknown parasitic components, $Z_{1}, Z_{2}, Z_{3}, G_{1}, G_{2}$, and $G_{3}$. The three-step de-embedding process is given in (B-1), (B-2), and (B-3).

$$
\begin{gathered}
\mathrm{Y}_{\mathrm{A}}=\mathrm{Y}_{\text {measur }}-\left[\begin{array}{cc}
G_{1} & 0 \\
0 & G_{2}
\end{array}\right] \\
\mathrm{Z}_{\mathrm{B}}=\mathrm{Z}_{\mathrm{A}}-\left[\begin{array}{cc}
Z_{1}+Z_{3} & Z_{3} \\
Z_{3} & Z_{2}+Z_{3}
\end{array}\right] \\
\mathrm{Y}_{D U T}=\mathrm{Y}_{\mathrm{B}}-\left[\begin{array}{cc}
G_{3} & -G_{3} \\
-G_{3} & G_{3}
\end{array}\right]
\end{gathered}
$$

The first step corrects for the influence of the admittances, $G_{1}$ and $G_{2}$. The second step de-embeds the effects of the series impedances, $Z_{1}, Z_{2}$, and $Z_{3}$. The third step removes the coupling $G_{3}$ between the two ports [66].

(b) Koolen's one-step de-embedding Method

The equivalent circuit of Koolen's de-embedding method is shown in Figure B-3. 


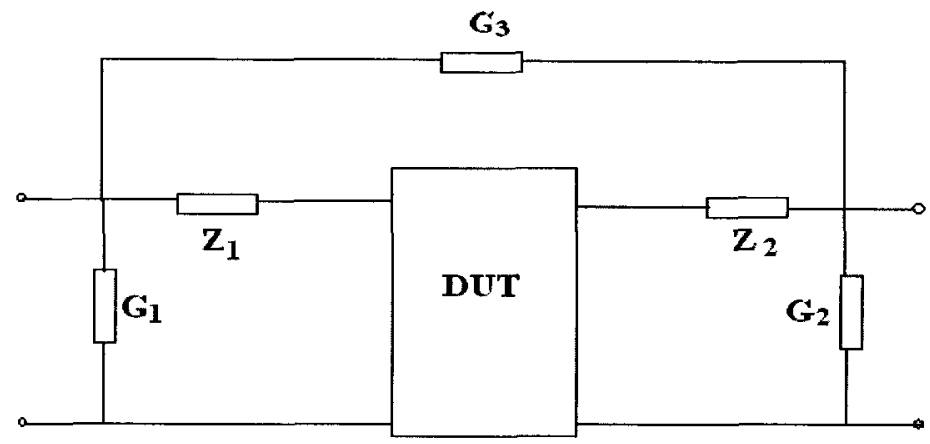

Figure B-3 Koolen's equivalent circuit of the RF device test structure.

In this method, only two de-embedding structures need to be built on the chip with the DUT: one is an open circuit; the other is a short circuit. The one-step de-embedding algorithm is given in (B-4). We can see that there is no need to determine the parasitic components, $Z_{1}, Z_{2}, G_{1}, G_{2}$, and $G_{3}$. The (B-4) formula only deals with $Y$-parameters directly from the measurements [68].

$$
Y_{D U T}=\frac{\left(Y_{\text {total }}-Y_{\text {open }}\right) \cdot\left(Y_{\text {short }}-Y_{\text {open }}\right)}{Y_{\text {short }}-Y_{\text {total }}}
$$




\section{Appendix C: X-parameters}

The theory of $X$-parameters is based on scattering functions, as are the $S$ parameters. But $X$-parameters are very different from $S$-parameters since they describe a nonlinear relationship in the frequency domain. The word 'scattering' refers to the fact that traveling-wave quantities are used in the theory. Here, 'traveling voltage waves' are represented by linear combinations of the signal port voltages and currents as defined in equation (C-1),

$$
\left(\begin{array}{l}
A \\
B
\end{array}\right)=\left(\begin{array}{l}
\frac{V+Z I}{2} \\
\frac{V-Z I}{2}
\end{array}\right) \text {. }
$$

The incident wave is represented by a capital $A$ and the scatted or reflected wave by a capital $B . V$ refers to the port voltage and $I$ to the port current, and $\mathrm{Z}$ to a chosen characteristic impedance.

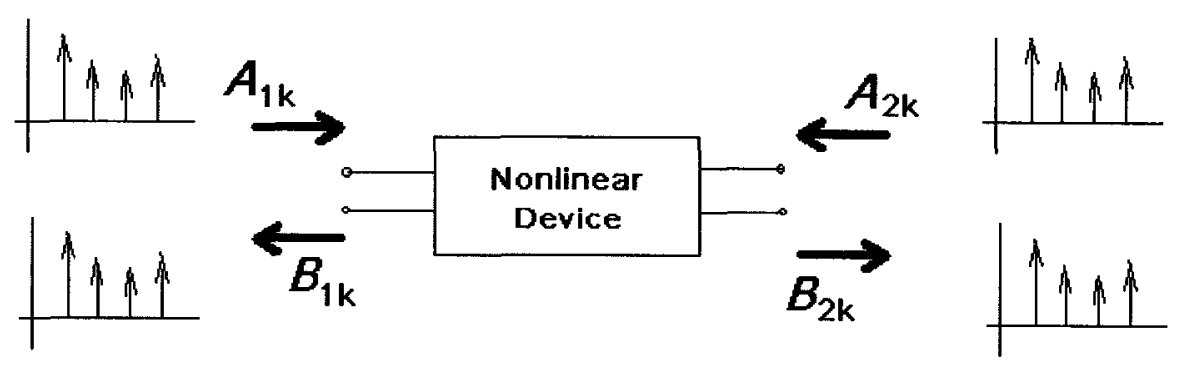

Figure C-1 Scattering notation for $\mathrm{X}$-parameters. 
Figure $\mathrm{C}-1$ gives more explanation of the theory. For the incident and reflected wave quantities, $A_{1 \mathrm{k}}, A_{2 \mathrm{k}}, B_{1 \mathrm{k}}$, and $B_{2 \mathrm{k}}$, the first subscript refers to the signal port and the second subscript to the harmonic index. Note that a harmonic index of 1 represents the fundamental component, a harmonic index of 2 refers the second harmonic, and so on. Using a general frequency-domain black-box modeling technique, the output spectral components, $B_{1 \mathrm{k}}$, and $B_{2 \mathrm{k}}$, correlate with the input spectral components, $A_{1 \mathrm{k}}$ and $A_{2 \mathrm{k}}$, and can be represented by the functions below:

$$
\begin{gathered}
B_{1 k}=F_{1 k}\left(A_{11}, A_{12}, \ldots ., A_{21}, A_{22}, \ldots \ldots .\right) \\
B_{2 k}=F_{2 k}\left(A_{11}, A_{12}, \ldots . . A_{21}, A_{22}, \ldots \ldots \ldots\right.
\end{gathered}
$$

where the letter $F$ denotes a correlation function.

In order to simplify the mathematics, phase-normalized quantities are used. That is, $A_{11}$ (the incident fundamental) is used as the phase-reference component. Since $A_{11}$ is the dominant large-signal input component, all other input components (at the harmonic frequencies) are relatively small compared to this dominant component. The mathematics of the phase normalization is quite simple. First, a component $P$ is defined such as that it has a phase equal to the phase of $A_{11}$ and unit amplitude of a unit: 


$$
P=e^{j p\left(A_{11}\right)}
$$

where $p\left(A_{11}\right)$ represents the phase of $A_{11}$.

The normalized quantities, denoted by the superscript $N$ are then calculated by multiplying the wave quantities by the reciprocal of $P$ raised to the power $k$, which corresponds to the harmonic index of that component.

$$
A_{m k}^{N}=A_{m k} P^{-k} \quad \text { and } \quad B_{m k}^{N}=B_{m k} P^{-k}
$$

Note that the phase-normalized quantity $A_{11}^{N}$ is a positive real number, that is $A_{11}^{N}=\left|A_{11}\right|$.

In general, the superposition principle and linear relationships are inapplicable to large-signal nonlinear systems. However, in many practical cases (e.g. narrow-band power amplifiers) there is only one dominant large-signal input component $\left(A_{11}\right)$ whereas all other input components are relatively small. In that case, it is valid to use the superposition principle for the relatively small phase-normalized input components. This is called the 'harmonic superposition' principle.

The harmonic superposition principle is graphically illustrated in Figure C-2. 


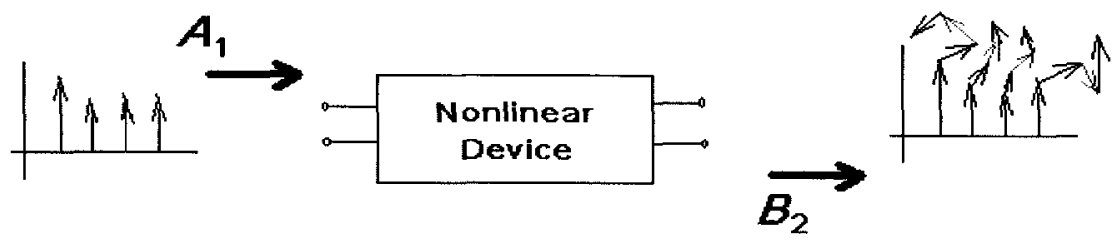

Figure C-2 The harmonic superposition principle.

In order to keep the graph simple only $A_{1}$ and $B_{1}$ are considered. First, assuming that $A_{11}$ is not zero, there will be a significant harmonic contribution to $B_{2}$. Now suppose that a relatively small harmonic component $A 12$ is present at the input. This will result in a change in the output spectrum $B_{2}$ at the second harmonic. The same principle holds for the third and fourth harmonics, and so on. The overall deviation of the output spectrum $B_{2}$ is the superposition of all the individual deviations. The 'harmonic superposition principle' is the key to the definition of the $X$-parameters. This principle leads to a very simple mathematical formulation of $X$-parameters, as shown in (C-6) [70].

$$
B_{m k}^{N}=\sum_{n k} X_{m k n k}\left(A_{11}^{N}\right) A_{n k}^{N}+X_{m k n k}^{\prime}\left(A_{11}^{N}\right) A_{n k}^{N^{*}}
$$

The phase-normalized $B^{\mathrm{N}}$ waves are the output quantities. They are written as a linear combination of the phase-normalized $A^{\mathrm{N}}$ waves, the input quantities, and their conjugates (denoted by the superscript ${ }^{*}$ ). Each $X$-function is defined by four subscripts: the first two denote the port and harmonic index of the associated output 
component $B$, and the latter two describe the port and harmonic index of the associated input component $A$.

The $X$-functions have some unique features compared to $S$-parameters. First, they relate input and output spectral components together at different frequencies. Second, they describe the fact that the ratio between the scattered $B^{\mathrm{N}}$ and incident $A^{\mathrm{N}}$ waves depends on the phase of the $A$ components relative to that of $A_{11}$. In general, $X$ parameters are nonlinear functions of the input signal power and can map incident signal at specific frequencies to other frequencies. Since $X$-parameters include many cross-frequency dependencies, there are more $X$-parameters than $S$-parameters. More information on $X$-parameters and the related large-signal testing are available in references [72], [73], and [74]. 


\section{References}

[1] H. Guckel, P. A. Brennan, and I. Palócz, "A Parallel-plate Waveguide Approach to Microminiaturized, Planar Transmission Lines for Integrated Circuits," IEEE Trans. Microwave Theory Tech., vol. 15, no. 8, pp. 468 -476, Aug. 1967.

[2] D. Jäger, "Slow-Wave Propagation Along Variable Schottky-Contact Microstrip Line," IEEE Trans. Microwave Theory Tech., vol. MTT-24, pp. 566 - 576, Sept. 1976.

[3] G. W. Hughes, and R. M. White, "MIS and Schottky Barrier Microstrip Devices," Proc. IEEE, vol. 60, no. 11, pp. 1460-1461, Nov. 1972.

[4] G. W. Hughes, and R. M. White, "Microwave Properties of Nonlinear MIS and SchottkyBarrier Microstrip," IEEE Trans. Electron Devices, vol. 22, ED-22, pp. 945-956, Oct. 1975.

[5] H. Hasegawa, and H. Okizaki, "MIS and Schottky Slow-wave Coplanar Striplines on GaAs Substrates," Electron. Lett., vol.13, pp. 663-664, Oct. 1977.

[6] M. Aubourg, J. P. Villotte, F. Godon, and Y. Garault, "Finite Element Analysis of Lossy Waveguides-Application to Microstrip Lines on Semiconductor Substrate," IEEE Trans. Microwave Theory Tech., vol. 31, pp. 326-331, Apr. 1983.

[7] Y. Fukuoka, Y. C. Shih, and T. Itoh, "Analysis of Slow-wave Coplanar Waveguide for Monolithic Integrated Circuits," IEEE Trans. Microwave Theory Tech., vol. 31, pp. 567-573, Apr. 1983.

[ 8] R. Sorrentino, G. Leuzzi, and A. Silbermann, "Characteristics of Metal-InsulatorSemiconductor Coplanar Waveguides for Monolithic Microwave Circuits," IEEE Trans. Microwave Theory Tech., vol. 32, pp. 410-416, Apr. 1984.

[9] D. Jäger, "Characteristics of Travelling Waves along the Non-linear Transmission Lines for Monolithic Integrated Circuits: a Review," International Journal of Electronics, vol. 58, pp. 649669, Apr. 1985.

[10] D. Jäger, "Soliton Propagation along Periodic-loaded Transmission Line," Appl. Phys., vol. 16, pp. 35-38, May 1978.

[11] M. E. Bastida, and G. P. Donzelli, "Periodic Slow-wave Low-loss Structures for Monolithic GaAs Microwave Integrated Circuits," Electron Lett., vol. 15, pp. 581-582, Sept. 1979.

[12] Y. Fukuoka, and T. Itoh, "Slow-wave Coplanar Waveguide on Periodically Doped Semiconductor Substrate," IEEE Trans. Microwave Theory Tech., vol. 31, pp. 1013-1017, May 1983.

[13] M. Dragoman, R. Kremer, and D. Jäger, "Pulse Generation and Compression on a Travelling-wave MMIC Schottky Diode Array", WRI International Conference, Ultra-wideband, Short-pulse Electromagnetic, Brooklyn, NY, pp. 67-74, Oct. 1992. 
[14] Ming Li, K. Krishnamurthi and R. G. Harrison, "A Fully Distributed Heterostructure-barrier Varactor Nonlinear-transmission Line Frequency Multiplier and Pulse Sharpener", IEEE Trans. Microwave Theory Tech. vol. 46, No. 12, pp. 2295 - 2301, Dec. 1998.

[15] U. D. Keil, and D. R. Dykaar, "Electrooptic Sampling at 150 fs," in Proc. 1993 OSA Conf. Ultrafast

Electron. Optoelectron., San Francisco, CA, pp. 25-27, Jan. 1993.

[16] Y. Ruai, M. Kamegawa, M. Case, M. Rodwell, and J. Franklin, "A 2.3-ps Time-domain Reflectometer

for Millimeter-wave Network Analysis," IEEE Microwave Guided Wave lett., vol. 1, pp. 334-336, Nov. 1991.

[17] D. W. Weide, "THz Spectroscopy Using Nonlinear Transmission Lines," Invited paper at IEEE International Workshop on Novel Terahertz Technology, Kobe, Japan, Nov. 1995.

[18] D. Salameh, and D. Linton, "Study of the Effect of Various Parameters on Nonlinear Transmission-Line (NLTL) Performance," IEEE Trans. Microwave Theory Tech., vol. 47, No. 3, pp. 350-353, Mar.1999.

[19] J. Rogers, and C. Plett, Radio Frequency Integrated Circuit Design, Boston: Artech House, 2003.

[20] M. J. W. Rodwell, S. T. Allen, R. Y. Yu, M. G. Case, U. Bhattacharya, M. Reddy, E. Carman, M. Kamegawa, Y. Konishi, J. Pusl, and R. Pullela, "Active and Nonlinear Wave Propagation Devices in Ultrafast Electronics and Optoelectronics," Proceedings of The IEEE, vol. 82, no. 7, pp. 1037-1059, July 1994.

[21] P. Wang, G. Pei, and E. C. C. Kan, "Pulsed Wave Interconnect," IEEE Trans. VLSI Systems, vol. 12 , no. 5, pp. 453-463, May 2004.

[22] J. M. Duchamp, P. Ferrari, M. Fernandez, A. Jrad, X. Melique, J. Tao, S. Arscott, D. Lippens, and R. G. Harrison, "Comparison of Fully Distributed and Periodically Loaded Nonlinear Transmission Lines," IEEE Trans. Microwave Theory Tech., vol. 51, no. 4, pp. $1105-$ 1116, April 2003.

[23] E. Afshari and A. Hajimiri, "Non-Linear Transmission Lines for Pulse Shaping in Silicon," IEEE J. Solid-State Circuits, vol. 40, no. 3, pp. 744-752, March 2005.

[24] F. Mohammed, M. F. Bain, F. H. Ruddell, D. Linton, H. S. Gamble, and V. F. Fusco, “A Novel Silicon Schottky Diode for NLTL Applications," IEEE Trans. Electron Devices, vol. 52, no. 7, pp. 1384-1391, July 2005.

[25] V. Milanovic, M. Ozgur, D. C. DeGroot, J. A. Jargon, M. Gaitan, and M. E. Zaghloul, "Characterization of Broad-Band Transmission for Coplanar Waveguides on CMOS Silicon Substrates," IEEE Trans. Microwave Theory Tech., vol. 46, no. 5, pp. 632-640, May 1998.

[26] H. Hasegawa, M. Furukawa, and H. Yanai, "Properties of Microstrip Line on Si-SiO2 System," IEEE Trans. Microwave Theory Tech., vol. 19, no. 11, pp. 869-881, May 1971. 
[27] A. C. Reyes, S. M. El-Ghazaly, S. Dorn, M. Dydyk, and D. K. Schroder, "Silicon as a Microwave Substrate," IEEE MTT-S Int. Microwave Symp. Dig., CA, pp. 1759-1762, San Diego, May 1994.

[28] S. Zaage, and E. Grotelueschen, "Characterization of the Broad-Band Transmission Behavior of Interconnections on Silicon Substrates," IEEE Trans. Comp., Hybrids, Manufact. Technol., vol. 16, no. 11, pp. 686-691, Nov. 1993.

[29] T. Shibata and E. Sano, "Characterization of MIS Structure Coplanar Transmission Lines for Investigation of Signal Propagation in Integrated Circuits," IEEE Trans. Microwave Theory Tech., vol. 38, no. 7, pp. 881-890, July 1998 .

[30] E. Tuncer and D. Neikirk, "Highly Accurate Quasi-Static Modeling of Microstrip Lines over Lossy Substrates," IEEE Microwave Guided Wave Lett., vol. 2, pp. 409-411, Oct. 1982.

[31] Y. R. Kwon, V. M. Hietala, and K. S. Champlin, "Quasi-TEM Analysis of 'Slow-Wave' Mode Propagation on Coplanar Microstructure MIS Transmission Lines," IEEE Trans. Microwave Theory Tech., vol. 35, no. 6, pp. 545-551, June 1987.

[32] B. Kleveland, T. H. Lee, and S. S. Wong, "50-GHz Interconnect Design in Standard Silicon Technology," IEEE MTT-S Int. Microwave Symp. Dig., vol. 3, pp. 1913-1916, Baltimore, June 1998.

[33] J. Kim, B. Jung, P. Cheung, and R. Harjani, "Novel CMOS Low-Loss Transmission Line Structure," IEEE Radio and Wireless Conference, pp. 235-238, Atlanta, Sept. 2004.

[34] G. E. Ponchak, A. Margomenos, and L. P. B. Katehi, "Low-Loss CPW on Low-Resistivity Si Substrates with a Micromachined Polyimide Interface Layer for RFIC Interconnects," IEEE Trans. Microwave Theory Tech., vol. 49, no. 5, pp. 866-870, May 2001.

[35] F. Krummenacher, M. Bucher, and W. Grabinsky, HF MOSFET Model Parameter Extraction, CRAFT European Project $N^{0}$ 25710, Lausanne, Switzerland, July, 2000.

[36] D. A. Johns and K. Martin, Analog Integrated Circuit Design, New York: John Wiley \& Sons, Inc. 1997.

[37] R. N. Simons, Coplanar Waveguide Circuits, Components and Systems, New York: Wiley Interscience, 2001.

[38] R. E. Collin, Foundations for Microwave Engineering, $2^{\text {nd }}$ ed. New York: McGraw-Hill, 1992. See Ch. 3, "Transmission lines and Waveguides".

[39] M. Y. Frankel, S. Gupta, J. A. Valdmanis, and G. A. Mourou, "Terahertz Attenuation and Dispersion Characteristics of Coplanar Transmission Lines", IEEE Trans. Microwave Theory Tech., vol. 39, no. 6, pp. 910-916, June 1991.

[40] R. K. Hoffmann, Handbook of Microwave Integrated Circuits, New York: Artech House, Inc., 1987. 
[41] F. Assadourian, and E. Rimai, "Simplified Theory of Microstrip Transmission Systems," Proceedings of the IRE, vol. 40, pp. 1651 - 1657, Dec. 1952.

[42] G. Hasnain, A. Dienes, and J. R. Whinnery, "Dispersion of Picosecond Pulses in Coplanar Transmission Lines," IEEE Trans. Microwave Theory Tech., vol. 34, no. 6, pp. 738 - 741, June 1986.

[43] http://www.sonnetsoftware.com/

[44] Advanced Design System User Guide, Agilent Technologies, Santa Clara, CA, 2003.

[45] B. Kleveland, C. H. Diaz, D. Vook, L. Madden, T. H. Lee, and S. S. Wong, "Exploiting CMOS Reverse Interconnect Scaling in Multigigahertz Amplifier and Oscillator Design," IEEE J. Solid-State Circuits, vol. 36, pp. 1480-1488, Oct. 2001.

[46] S. N. Lee, Y.J. Kim, and J. G. Yook, "Design and Characterization of Low-loss and Lowdispersion Micromachined Elevated CPW," Microwave and Optical Technology Lett., vol. 39, No. 3, pp.169-171, Mar. 2003.

[47] J. Kim, B. Jung, P. Cheung, and R. Harjani, "Novel CMOS Low-loss Transmission Line Structure," IEEE Radio and Wireless Conference, pp. 235-238, Atlanta, Sept. 2004.

[48] T. S. D. Cheung, J. R. Long, K. Vaed, R. Volant, A. Chinthakindi, C. M. Schnabel, J. Florkey, and K. Stein, "On-Chip Interconnect for Mm-Wave Applications Using an All-Copper Technology and Wavelength Reduction," Proc. IEEE ISSCC, pp. 396 - 397, San Francisco, Feb. 2003.

[49] R. E. Amaya, M. Li, R. G. Harrison and N. G. Tarr, "Coplanar Waveguides in Silicon with Low Attenuation and Slow Wave Reduction," Proceedings of the 37th European Microwave Conference, pp. 508-511, Munich, Germany, October 2007.

[50] Ansoft HFSS, Available online: http://www.ansoft.com/products/hf/hfss/.

[51] R. L. Bunch, and S. Raman, "Large-Signal Analysis of MOS Varactors in CMOS $-G_{\mathrm{m}} L C$ VCOs," IEEE J. Solid-State Circuits, vol. 38, no. 8, pp. 1325-1332, Aug. 2003.

[52] Medici User Manual, Synopsis Inc., 2003.

[53] S. Thompson, P. Packan, and M. Bohr, "Mos Scaling: Transistor Challenges for $21^{\text {st }}$ Century," Intel Technology Journal, Q3, pp. 1-19, Sept. 1998.

[54] A. S. Grove, Physics and Technology of Semiconductor Devices, New York: John Wiley \& Sons, 1976.

[55] S. K. Banerjee, Y. Du, R. Thoma, A. C. Duvallet, "Simulation and Benchmarking of MOS Varactors for CMOS090 RF Process," Motorola $S^{3}$ Symposium, pp. 1-10, Itasca, Illinois, July 2003.

[56] J. S. Dunn, D. C, Ahlgren, D. D. Coolbaugh, N. B. Feilchenfeld, G. Freeman, D. R. Greenberg, R. A. 
Groves, F. J. Guarin, Y. Hammad, A. J. Joseph, L. D. Lanzerotti, S. A. St.Onge, B. A. Orner, J. S. Rieh, $\mathrm{K}$.

J. Stein, S. H. Voldman, P. C. Wang, M. J. Zierak, S. Subbanna, D. L. Harame, D. A. Herman, Jr. and B. S.

Meyerson, "Foundation of RF CMOS and SiGe BiCMOS technologies," IBMJ. Res. \& Dev., vol. 47, no. 2-

3, pp. 101-138, March-May 2003.

[57] B. Razavi, “A 60-GHz CMOS Receiver Front-End,” IEEE J. Solid-State Circuits, vol. 41, no. 1, pp. 17-22, Jan. 2006.

[58] C. Cao, and K. K. O, "Millimeter-Wave Voltage-Controlled Oscillators in 13- $\mu$ m CMOS Technology," IEEE J. Solid-State Circuits, vol. 41, no. 6, pp. 1297-1304, June 2006.

[59] P. Sameni, C. Siu, S. Mirabbasi, H. Djahanshahi, M. Hamour, K. Iniewski, and J. Chana, "Modeling and Characterization of VCOs with MOS Varactors for RF Transceivers," Hindawi Publishing Corporation, EURASIP Journal on Wireless Communications and Networking, vol. 2006, pp. 1-12, Jan. 2006.

[60] D. Dunwell, and B. Frank, "Accumulation-Mode MOS Varactor for RF CMOA Low-Noise Amplifiers," IEEE Topical Meeting on Silicon Monolithic Integrated Circuits in RF Systems (SiRH07), pp. 145-148, Long Beach CA, Jan. 2007.

[61] A. Jrad, P. Ferrari, C. Fuchs, A. Dominjon, J.W. Tao, B. Flechet, and G. Angenieux, "A Simple and Systematic Method for the Design of Tapered Nonlinear Transmission Lines," IEEE MTT-S Int. Microwave Symp. Dig., pp. 1627-1630, Baltimore, Jun. 1998.

[62] G. Bartolucci, F. Pini, R. Marcelli, M. Dragoman, and S. Simion, "Second Harmonic Generation by Means of Double Tapered Non-linear Transmission Line," Gallium Arsenide Application Symposium (GAAS), pp. 4-7, Paris, June 1996.

[63] H. J. Eul and B. Schiek, "A Generalized Theory and New Calibration Procedures for Network Analyzer Self-Calibration," IEEE Trans. on Microwave Theory and Techniques, vol. 39, pp. 724-731, April 1991.

[64] P. J. Van Wijnen, H. R. Claessen, and E. A. Wolsheimer, "A New Straightforward Calibration and Correction Procedure for On-wafer High Frequency $S$-parameters Measurements (45 MHz-18 GHz)," Proceedings of IEEE Bipolar Circuits and Technology Meeting, pp. 91-94, Minneapolis, Sept. 1988.

[65] A. Fraser, R. Gleason, and E. W. Strid, "GHz On-Silicon-Wafer Probing Calibration Methods," Proceedings of IEEE 1988 Bipolar Circuits and Technology Meeting, pp. 154-157, Minneapolis, Sept. 1988.

[66] E. P. Vandamme, D. Schreurs, and G. Van Dinther, "Improved Three-step De-embedding Method to Accurately Account for the Influence of Pad Parasitics in Silicon On-wafer RF TestStructures," IEEE Trans. Electron Devices, vol. 48, no. 4, pp. 737-742, April 2001.

[67] H. Cho et al., "A three-step method for the de-embedding of high frequency S-parameter measurements", IEEE Trans. Electron Devices, vol. 38, no. 6, pp. 1371-1375, Jun. 1991. 
[68] M. Koolen, J. Geelen, and M. Versleijen, "An Improved De-embedding Technique for Onwafer High-Frequency Characterization," Proceedings of the IEEE Bipolar Circuits and Technology Meeting, pp. 188-191, Santa Barbara, Aug. 1991.

[69] P. Heymann, H. Prinzler, and F. Schnieder, "De-embedding of MMIC transmission-line measurements", IEEE MTT-S International Microwave Symposium Digest, vol. 2, pp. 10451048, San Diego, May 1994.

[70] J. Wood, and D. E. Root, Fundamentals of Nonlinear Behavior Modeling for RF and Microwave Circuits, Boston: Artech House, 2005. See chapter 5.

[71] Available online at: http//www.eesof.tm.agilent.com/products/ads2008.html

[72] D. E. Root et al., "Broad-Band Poly-Harmonic Distortion (PHD) Behavioral Models from Fast Automated Simulations and Large-Signal Vectorial Network Measurements," IEEE Trans. on Microwave Theory and Tech., vol. 53, No. 11, pp. 3656-3664, Nov. 2005.

[73] J. Verspecht et al., "Multi-tone, Multi-Port, and Dynamic Memory Enhancements to PHD Nonlinear Behavioral Models from Large-Signal Measurements and Simulations," IEEE MTT-S International Microwave Symposium Digest, pp. 969-972, Honolulu, June 2007.

[74] J. Horn et al. "Measurement-Based Large-signal Simulation of Active Components from Automated Nonlinear Vector Network Analyzer Data via X-parameters," IEEE COMCAS (Microwaves, Communications, Antennas and Electronic Systems) International Conference, pp. 1-6, Tel-Aviv, May 2008.

[75] Available online at: www.cascademicrotech.com

[76] B. Schiek, "Developments in Automatic-Network Analyser Calibration Methods," in Review of Radio

Science 1993-1996, Oxford Science Publications: pp. 115-155, Oxford, 1996.

[77] H. J. Eul and B. Schiek, "Thru-Match-Reflect: One Result of A Rigorous Theory for Deembedding and Network Analyzer Calibration," in 18th European Microwave Conference Digest, pp. 909-914, Stockholm, Sept. 1988. 